\begin{abstract}
BALANCING PARTICLE ABSORPTION WITH STRUCTURAL SUPPORT OF THE MUON BEAM STOP IN MUONS-TO-ELECTRONS EXPERIMENTAL CHAMBER

\author{
Ryan Majewski, M.S. \\ Department of Mechanical Engineering \\ Northern Illinois University, 2013 \\ Nicholas Pohlman, Director
}

The Mu2e experiment at Fermi National Accelerator Laboratory is structured to discover a full conversion from muon to electron. This discovery could explain some of the most sought-out questions in high energy particle physics today. The experiment will utilize high energy protons to create a muon beam, by utilizing various boosters and accelerators. During the experiment, radioactive particles will be created. It is important that certain components inside the experiment and the outside world are shielded from particles such as neutrons, electrons, and photons. The muon beam stop (MBS) is one of the main components that is responsible for this type of shielding. The MBS is a cylindrical assembly of 316L stainless steel and high density polyethylene that sits atop a support structure on rolling bearing blocks that maintain alignment. The two main priorities are to improve the particle absorption of the currently designed MBS and ensure that it can be supported from a structural standpoint. For the particle physics, simulations were run using Muons, Inc.'s G4Beamline environment, where the geometry of the MBS was varied to see the impact on particle absorption. Subsequent structural and geometric analysis was done to confirm the 
new geometries are mechanically viable. In the end, a refined MBS was found that can be supported safely within the experimental constraints. 
NORTHERN ILLINOIS UNIVERSITY

DE KALB, ILLINOIS

MAY 2013

\title{
BALANCING PARTICLE ABSORPTION WITH STRUCTURAL SUPPORT OF THE MUON BEAM STOP IN MUONS-TO-ELECTRONS EXPERIMENTAL CHAMBER
}

\author{
BY \\ RYAN MAJEWSKI \\ (C) 2013 Ryan Majewski
}

\begin{abstract}
A THESIS SUBMITTED TO THE GRADUATE SCHOOL IN PARTIAL FULFILLMENT OF THE REQUIREMENTS

FOR THE DEGREE

MASTER OF SCIENCE
\end{abstract}

DEPARTMENT OF MECHANICAL ENGINEERING

Thesis Director:

Nicholas Pohlman 


\section{ACKNOWLEDGEMENTS}

First, I would like to thank the Mu2e Collaboration and director Ron Ray at Fermilab for there funded support throughout this project. I would also like to thank my thesis advisor, Dr. Nicholas Pohlman, for giving me the opportunity to work on this project. His insight, guidance, and suggestions enabled me to effectively conduct my research. Likewise, Dr. David Hedin provided the particle physics background knowledge and support which I am grateful for. Without his resources I would not have been able to complete my studies. Another very useful resource was Zachary Hodge, a Master's student in the Department of Physics at Northern Illinois University. I accredit him with much of the initial work that helped me become comfortable with running G4Beamline simulations, and helping me to write my setup and macro files. Dr. Meung Kim's help with finite element analysis was second to none. The engineers and physicists at Fermilab were extremely helpful in providing me the proper information for my studies, in particular Giuseppe Gallo and Rodger Bossert.

Finally, I would like to thank my family and many of my friends. My brother, Kyle Majewski, and sister, Hannah Majewski were always there to provide an oasis away from my research. Similarly, my granparents, David and Janet Syens, were always supportive of my higher education aspirations. Likewise, without the late night study partners, Zach Rio, Rusty Dean, and Haitham Felemban, this thesis might still be in progress. Also, Amanda Krutz was the most supportive person of my studies, always pushing me to do more or run one more simulation when I did not want to, for this I am forever thankful. 


\section{DEDICATION}

To my mother and father, Kurt and Sherry Majewski, for their unconditional support and unparalleled parenting. 


\section{TABLE OF CONTENTS}

Page

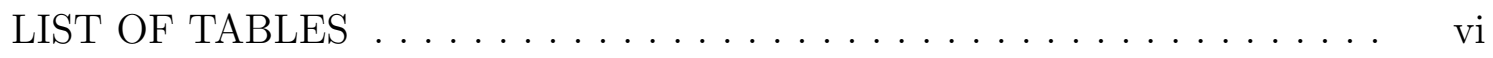

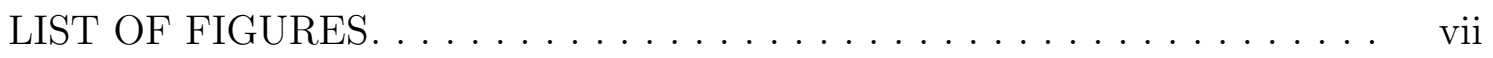

LIST OF APPENDICES ..................... ix

Chapter

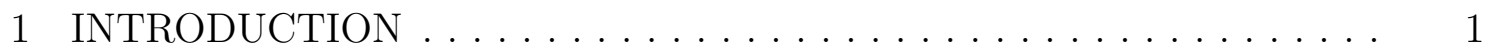

1.1 What is a Muon? ....................... 1

1.2 Mu2e Experiment Overview . . . . . . . . . . . . . . . 2

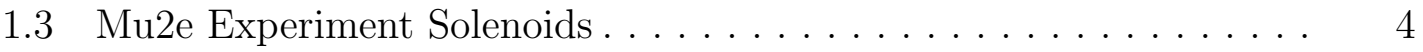

1.3.1 Production Solenoid $(\mathrm{PS}) \ldots \ldots \ldots \ldots \ldots$

1.3.2 Transport Solenoid $(\mathrm{TS}) \ldots \ldots \ldots \ldots \ldots$

1.3.3 Detector Solenoid $(\mathrm{DS}) \ldots \ldots \ldots \ldots \ldots$

1.4 Mu2e Experiment Timeline . . . . . . . . . . . . . . 8

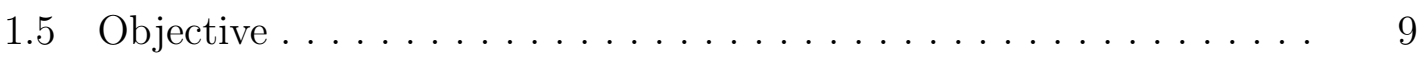

2 BACKGROUND OF PRELIMINARY MUON BEAM STOP DESIGN . . 11

2.1 Characteristics of the Muon Beam Stop . . . . . . . . . . . . . . 11

2.2 Proposed Installation and Previous Analysis . . . . . . . . . . . . . 14

2.3 Goals and Constraints . . . . . . . . . . . . . . . . . . . . . 19

3 SIMULATIONS IN G4BEAMLINE ENVIRONMENT . . . . . . . . . . . . . 22

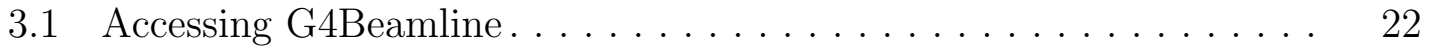

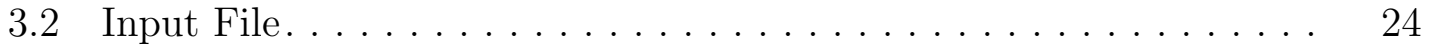


Chapter $\quad$ Page

3.3 Geometry Files...................... 26

3.4 Running a Simulation . . . . . . . . . . . . . . . . 27

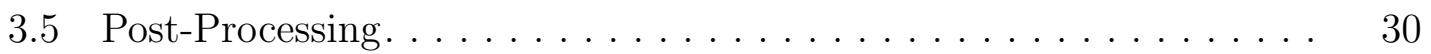

4 G4BEAMLINE SIMULATION RESULTS . . . . . . . . . . . . . . . 33

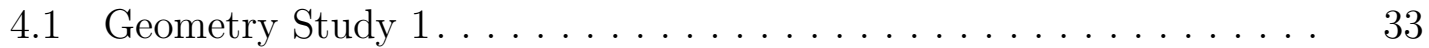

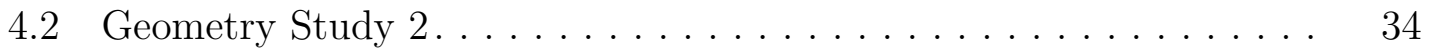

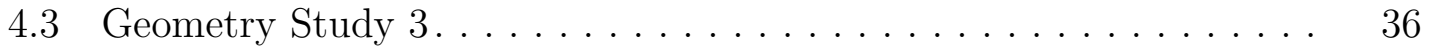

4.4 Geometry Studies 4,5 , and $6 \ldots \ldots \ldots \ldots \ldots \ldots$

4.5 Additional Studies and Conclusions . . . . . . . . . . . . . 42

5 ENGINEERING CONSIDERATIONS . . . . . . . . . . . . . . . . . 47

5.1 New MBS Measurables . . . . . . . . . . . . . . . . 47

5.2 Support Structure. . . . . . . . . . . . . . . . . 49

5.3 Possible Assembly Procedures . . . . . . . . . . . . . . . . 52

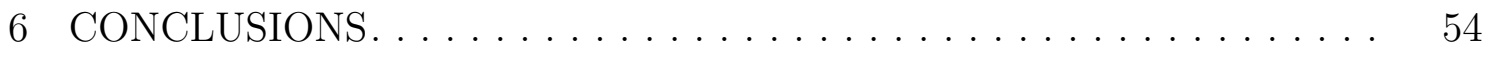

6.1 An Improved $\mathrm{MBS} \ldots \ldots \ldots \ldots \ldots \ldots \ldots \ldots$

6.2 Future Work. . . . . . . . . . . . . . . . . . 55

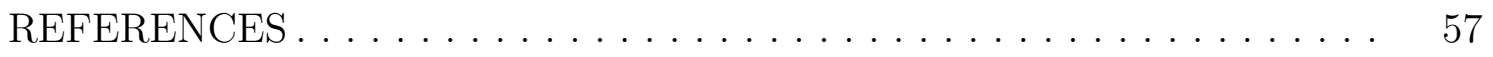

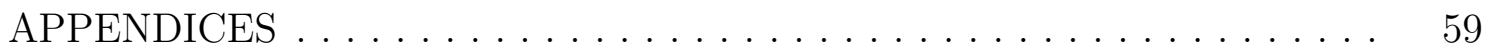




\section{LIST OF TABLES}

Table

Page

1.1 Mu2e Timeline $[1] \ldots \ldots \ldots \ldots \ldots \ldots \ldots \ldots$

2.1 Material Properties of MBS Materials [9] . . . . . . . . . . . . . 12

2.2 Dimensions and Mass of Current MBS Components [9] . . . . . . . 13

2.3 THK Bearing Block Load Ratings $(\mathrm{N})[11] \ldots \ldots \ldots \ldots \ldots$

4.1 Study 1 Results. . . . . . . . . . . . . . . . . . . . . 34

4.2 Study 1 Percentage Change . . . . . . . . . . . . . . . . . . 34

4.3 Study 2 Results. . . . . . . . . . . . . . . . . . . 35

4.4 Study 2 Percentage Change . . . . . . . . . . . . . . . 35

4.5 Study 3 Results. . . . . . . . . . . . . . . . . . 36

4.6 Study 3 Percentage Change . . . . . . . . . . . . . . . . . 37

4.7 Study 4 Results. . . . . . . . . . . . . . . . . . . . 38

4.8 Study 4 Percentage Change . . . . . . . . . . . . . . . . . 39

4.9 Study 5 Results. . . . . . . . . . . . . . . . . . . . . 39

4.10 Study 5 Percentage Change . . . . . . . . . . . . . . . . . . 40

4.11 Study 6 Results. . . . . . . . . . . . . . . . . . . . . . 40

4.12 Study 6 Percentage Change . . . . . . . . . . . . . . . . . . 41

4.13 Final MBS Geometry Simulation Results. . . . . . . . . . . . . . . 45 


\section{LIST OF FIGURES}

$\begin{array}{lll}\text { Figure } & \text { Page }\end{array}$

1.1 Fermilab's Mu2e Layout $[1] \ldots \ldots \ldots \ldots \ldots \ldots$

1.2 Solenoids on the Muon Campus $[1] \ldots \ldots \ldots \ldots \ldots$

1.3 The 5 Sections of the Transport Solenoid $[7] \ldots \ldots \ldots$

1.4 The Detector Solenoid $[8] \ldots \ldots \ldots \ldots \ldots \ldots \ldots$

2.1 Components of the MBS $[9] \ldots \ldots \ldots \ldots \ldots \ldots \ldots \ldots$

2.2 MBS Supported $[9] \ldots \ldots \ldots \ldots \ldots \ldots \ldots \ldots$

2.3 Detector Solenoid Components Installation $[10] \ldots \ldots \ldots \ldots \ldots$

2.4 Section View of Assembled Detector Solenoid [10] . . . . . . . . . 16

2.5 Internal Rail System $[11] \ldots \ldots \ldots \ldots \ldots$

2.6 FEA for Internal Rail Bearing Blocks $[11] \ldots \ldots \ldots \ldots \ldots$

2.7 Deflection Along the Y-axis of the MBS Support Structure [12] . . . 18

2.8 MBS Expandable Space. . . . . . . . . . . . . . . . . 20

3.1 Accessing XWin Through the Cygwin Terminal . . . . . . . . . . . . 22

3.2 Accessing detsim.fnal.gov Through Xwin . . . . . . . . . . . . 23

3.3 Detsim User Home Directory . . . . . . . . . . . . . . . . . . . 24

3.4 Command to Source the Setup File. . . . . . . . . . . . . . . . 27

3.5 Running a G4Beamline Simulation . . . . . . . . . . . . . . 28

3.6 Outputs to XWin Window. . . . . . . . . . . . . . . . 29

3.7 Command to Start the Viewer in G4Beamline . . . . . . . . . . . . 29 
Figure $\quad$ Page

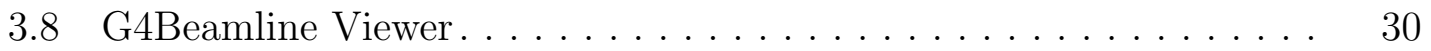

3.9 Starting Root and Macro . . . . . . . . . . . . . . . . . . 31

3.10 Example Output from the Macro . . . . . . . . . . . . . . . . . . 32

4.1 Geometry Definition for Studies 4,5 , and $6 \ldots \ldots \ldots$

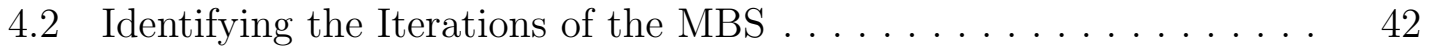

4.3 Layered 316L Stainless Steel and Polyethylene MBS. . . . . . . . . . . 43

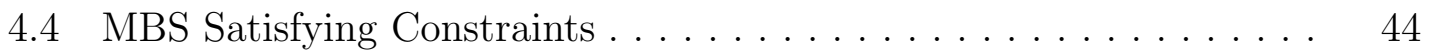

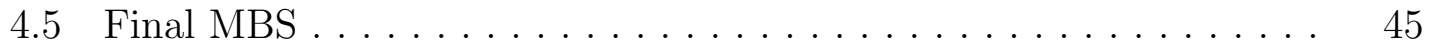

5.1 Outer Dimensions of the New MBS. . . . . . . . . . . . . . 48

5.2 MBS with IFB Support Pins . . . . . . . . . . . . . . . . . 49

5.3 Stress Analysis on IFB Support Pins $[14] \ldots \ldots \ldots$. . . . . . . . . . 50

5.4 Deflection Analysis on Main Stainless Steel Tube. . . . . . . . . . . 50

5.5 Deflection Analysis with Stiffener Rings . . . . . . . . . . . . . . 51 


\section{LIST OF APPENDICES}

$\begin{array}{lll}\text { Appendix } & \text { Page }\end{array}$

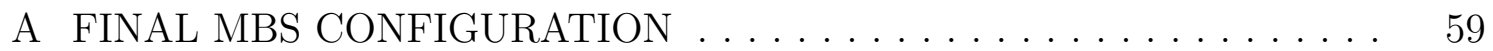

B G4BEAMLINE INPUT FILE . . . . . . . . . . . . . . . . . . . . . . 61

C G4BEAMLINE MBS GEOMETRY FILE . . . . . . . . . . . . . . . . . . 75

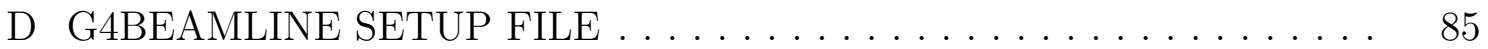

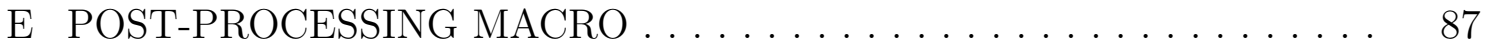




\section{CHAPTER 1 INTRODUCTION}

The Mu2e experiment at Fermi National Accelerator Laboratory is seeking a full conversion from muon to electron. The design for Mu2e is based off MECO, another proposed experiment that sought a full conversion from muon to electron at Brookhaven National Laboratory in the 1990s. Mu2e will provide sensitivity that is

four times the sensitivity of the previous experiment, SINDRUM II [1]. Discovering muon to electron conversions could help explain physics beyond the standard model of the particle physics.

\subsection{What is a Muon?}

The three types of elementary particles are quarks, leptons, and bosons. Of these three types, the muon is one of the three charged leptons, along with the tau, and the well known electron. The muon is very similar to the electron because of its negative charge, but different in that the muon is heavier than the electron. In fact, the muon is over 200 times heavier than the electron [2]. It has been observed that members within the quark and lepton families are able to change into other types of quarks or leptons, respectively, within the family [3]. This phenomena has never been observed for a muon to electron, within the lepton family, and this is sought out within the physics community. A conversion from muon to electron could provide new insight to the world of particle physics. 
If the conversion from muon to electron is found it would show that there is new physics beyond the current understanding and this would occur at sensitivities better than what can be seen at CERN's large hadron collider [3]. As well it would provide insight on other topics in particle physics like symmetry [4]. Symmetry is an important part of particle physics because symmetry principles impact reproducibility in physical events [5]. While this discovery would show that there is new physics beyond the current understanding, it would also open up new questions. Some of these new questions include the following [4]:

- What are the forces that affect particle interactions at high energies?

- Why are there so many particles?

- Is the conversion from muon to electron related to the quark conversion?

Discovering this conversion would be beneficial for the scientific community moving ahead. It would confirm past thoughts about the physical laws, such as the muons to electron conversion, while opening new doors for research and development on the standard model.

\subsection{Mu2e Experiment Overview}

Mu2e will utilize Fermilab's existent particle accelerator, as well it will construct its own beamline for the experiment. The main components of the experiment are the recycler ring, debuncher ring, and the Muon campus. The Muon campus is the site for the newly constructed beamline. The recycler and debuncher rings will accelerate protons to a high energy state and extract them to the Muon campus in order to provide the muon beam needed for the experiment [1]. 


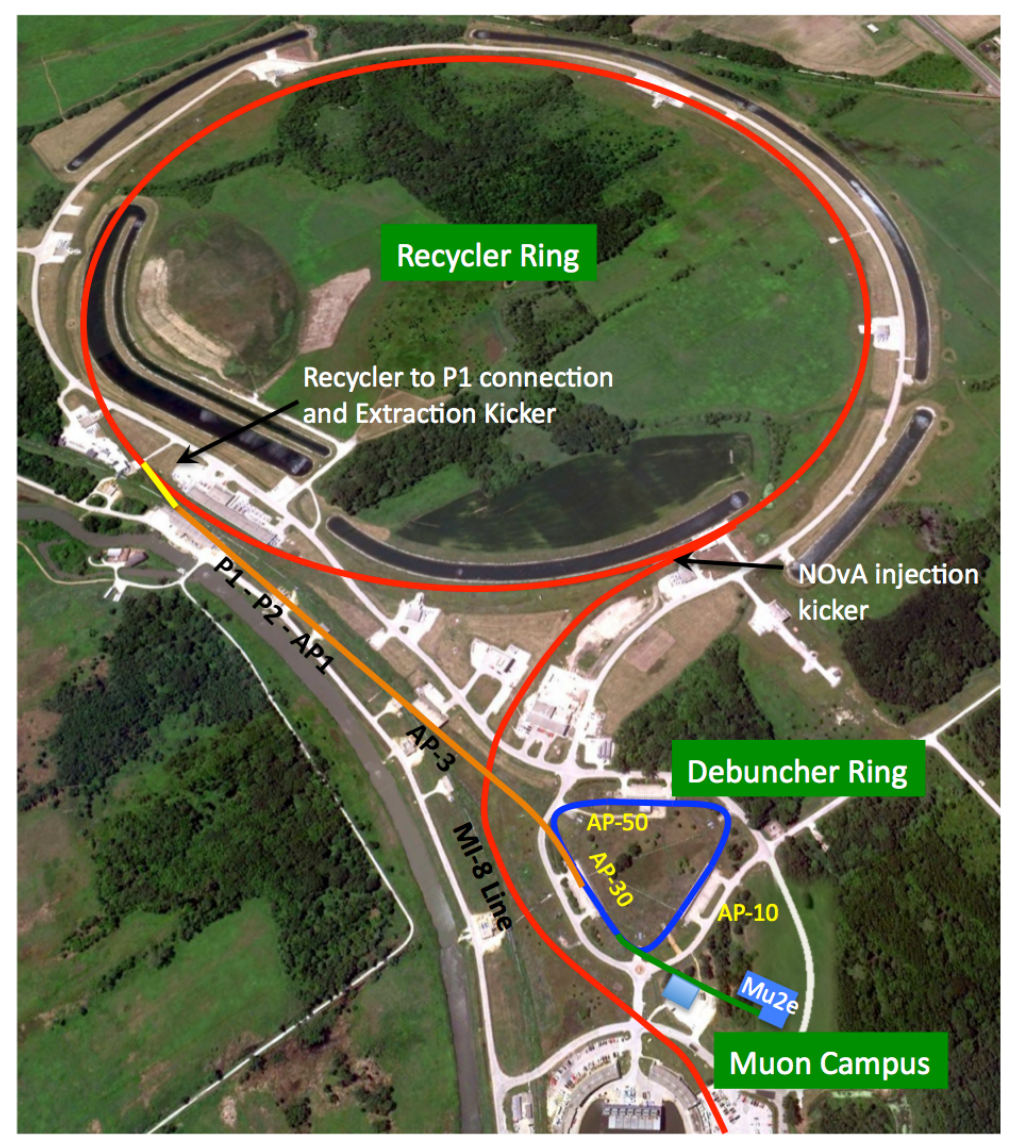

Figure 1.1: Fermilab's Mu2e Layout [1]

This experiment requires a high intensity proton beam in order to produce the needed muon beam [1]. The protons will start by being boosted through the MI8 beamline to the recycler ring where they will be re-bunched. These re-bunched protons will then circle the recycler ring, be split into four smaller bunches, and then exit at the P1 line which connects the recycler ring to the debuncher ring. Once in the debuncher ring the protons will be extracted to the Muon campus in a pulsed fashion. By sending the protons to the Muon campus this way, it allows some of the first batch particles to decay before the next batch is sent to the Muon campus. These bunches are separated by the revolution period of the Debuncher Ring, which is about $1.7 \mu \mathrm{s}$ [1]. Figure 1.1 shows the layout of the Mu2e experiment. Once in the 
Muon campus, the particles will travel through 3 different solenoids, the production, transport, and detector solenoids, in order to complete the experiment.

\subsection{Mu2e Experiment Solenoids}

The Mu2e experiment will contain three solenoids. These solenoids will take the incoming protons and produce muons that will be transported to detect the conversion from muons to electron. Figure 1.2 shows the layout of the three solenoids contained within the experiment.

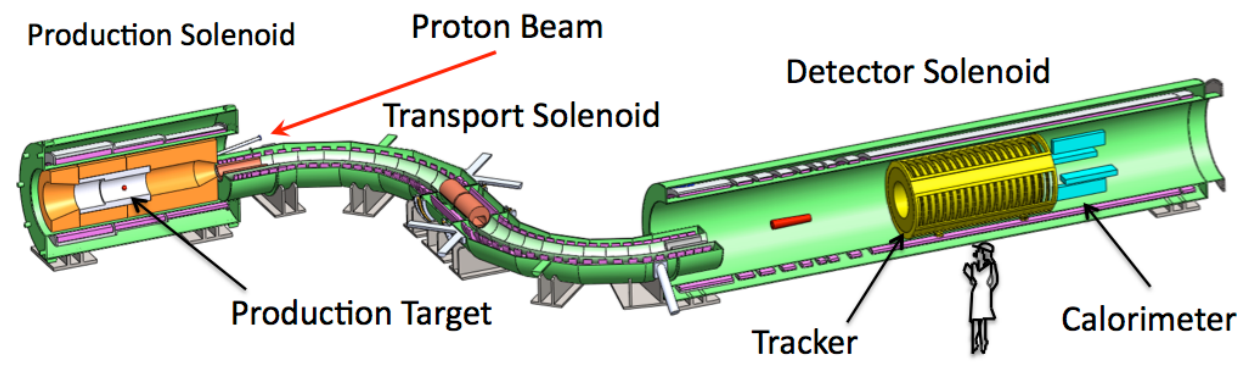

Figure 1.2: Solenoids on the Muon Campus [1]

It can be seen that the protons will first enter and go through the production solenoid (PS), where they hit the production production target. Then some particles produced in the target travel to the detector solenoid (DS) region through the transport solenoid (TS). Some muons are then stopped in the DS region and are used to search for their direct conversions to electrons. All three of these solenoids play a crucial role in the experiment for various reasons. 


\subsubsection{Production Solenoid (PS)}

The production solenoid (PS) is the first solenoid that the incoming particles will see. The main function of the PS is to provide muons for the experiment. The muons are created by directing the primary proton beam into the production target located in the PS, which can also be seen in Figure 1.2 [6]. The PS will maximize the muon yield by directing the muons toward the transport solenoid, while keeping these muons within the appropriate momentum range by supplying the correct magnetic field [6]. The PS provides space for beamline components, such as the production target. Another function of the PS is to provide secondary particle radiation protection. Finally, the PS allows the primary proton beam to exit the PS without interfering with the PS magnet shield, which is highlighted by a red arrow in Figure $1.2[6]$.

\subsubsection{Transport Solenoid (TS)}

The transport solenoid (TS) is the next solenoid to see the incoming particles. Just like its name, the TS is responsible for transporting the muons toward the detector solenoid's stopping target. The TS will maximize this muon yield by directing the muons that are within the proper momentum range toward the detector solenoid's stopping target [7]. A unique feature about the TS is its S-shape, which can be seen in Figure 1.3.

The straight sections of the TS are TS1, TS3, and TS5, while the curved sections are TS2 and TS4. The magnetic field in TS1 is matched to the field in the PS for the best transmission of particles form solenoid to solenoid. Similarly, the magnetic 


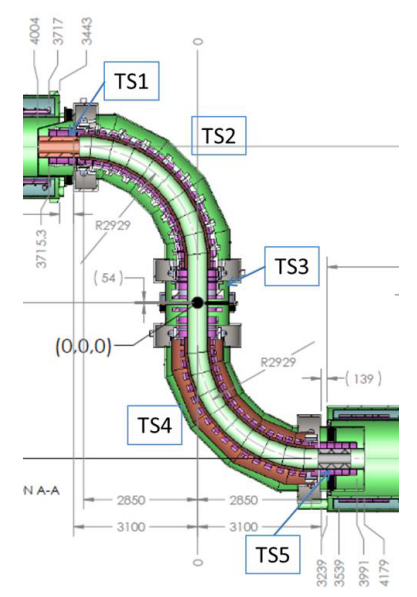

Figure 1.3: The 5 Sections of the Transport Solenoid [7]

field of TS5 is match to the field of the detector solenoid for optimal transmission to the stopping target [7]. All of the straight sections prevent the particles traveling through from losing longitudinal momentum while going through the TS. The first curved section, TS2, disperses the beam in order for a collimator to do a sign and momentum selection of the particles [7]. Finally, the second curved section, TS4, aligns the center of the muon beam to the center of TS5, which aligns the muon beam to the stopping target in the detector solenoid [7]. The scatter and random energy of the original production particles is now carefully selected for observing the muon to electron conversion.

\subsubsection{Detector Solenoid (DS)}

The final solenoid that the particles will see is the detector solenoid (DS). The main responsibilities of the DS are to maximize the muon yield and focus these muons toward the stopping target. Likewise, the DS uses the magnetic field to measure the electron momentum in the front half, and the fall off of the field in the 
back half allows muons to move to the sides of the muon beam stop. About $40 \%$ of the incoming muons will come to rest in the aluminum stopping target and be captured by the nucleus. They will subsequently either decay in orbit or undergo nuclear capture with a very small fraction possibly undergoing direct conversion. The remaining $60 \%$ of the muons either stop in the muon beam stop (MBS) or go through the hole in the MBS to the target stopping monitor. The energies of the electrons will be measured by tracking elements while a calorimeter will be used for particle identification [8]. The components located within the DS bore, including the target tracker and calorimeter, can be seen in Figure 1.4. These two instruments will be able to tell whether or not when a muon strikes the production target if it decays into an electron.

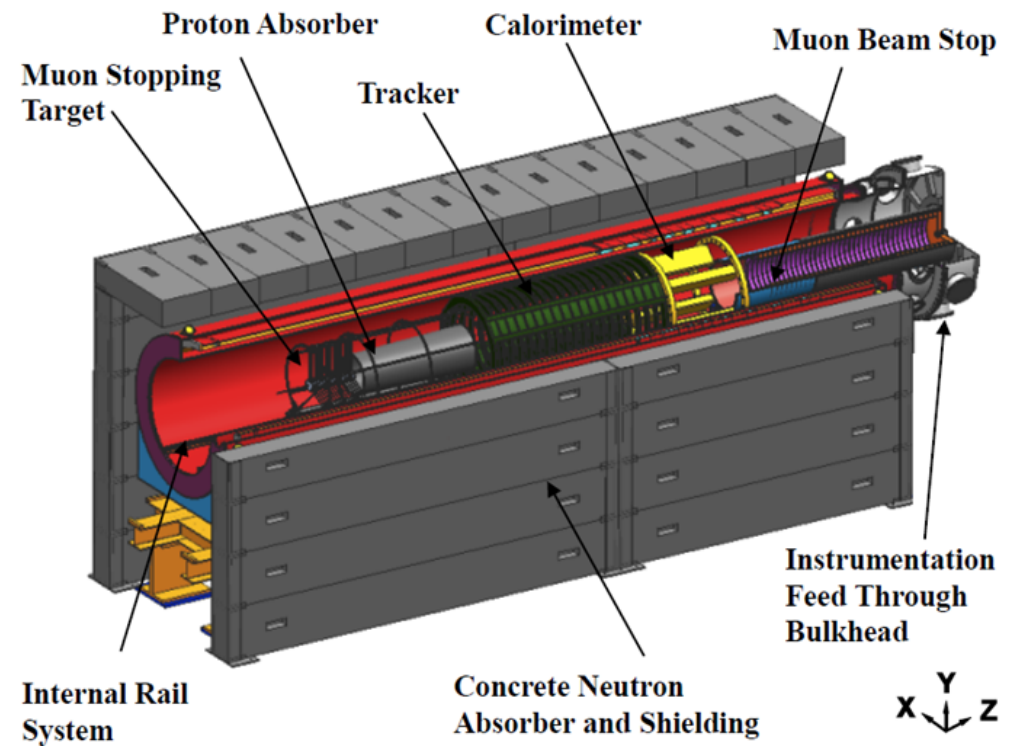

Figure 1.4: The Detector Solenoid [8]

The DS has components within that act as shielding device to protect the solenoid from primary and secondary beam radiation [8]. One of the main components within the DS that will be used for shielding is the muon beam stop (MBS). As can be seen in Figure 1.4, the MBS is located at the end of the DS. This com- 
ponent is a multi-material cylindrically shaped beam stop that is intended to shield components within the experiment and the outside world from radiation.

The MBS is crucial from the aspect of shielding. This component is key in terms of controlling the backscatter of electrons and photons into the target tracker and calorimeter. Minimizing this backscatter provides more accurate means of measurement, because it will prevent the target tracker and/or the calorimeter from counting an electron more than once. Likewise, the MBS dealt with the task of absorbing neutrons, which are an appreciable background in the cosmic ray veto counters located outside the DS. Finally, there is currently a $101 \mathrm{~mm}$ diameter hole at the end of the MBS which provides a window for the remaining muon beam to exit through. The positioning of this hole is crucial to the experiment, which calls for a positional accuracy of $+/-2 \mathrm{~mm}[9]$.

\subsection{Mu2e Experiment Timeline}

Mu2e is a very large scale experiment that requires a lot of planning and scheduling. Organizing an experiment of this magnitude requires several performance milestones to be hit in order for the experiment to be executed. The plan for Mu2e is

broken down into several stages. Currently, Mu2e is in conceptual design stage 1, CD-1, and is preparing for CD-2. In order for Mu2e to move from CD-1 into CD-2, there has to be a finalized and approved conceptual design for the experiment. Evaluation of feasibility in the building and operation of the experiment are required in order to achieve CD-2 status. During CD-2, the final designs are approved resulting in CD-3 where construction can commence. 
Table 1.1: Mu2e Timeline [1]

\begin{tabular}{lc}
\hline \hline Major Milestone Events & Preliminary Schedule \\
\hline CD-0 (Approve Mission Need) & 1st Qtr.,FY10 \\
CD-1 (Approve Alternate Selection/Cost Range) & 4th Qtr.,FY12 \\
CD-2 (Approve Performance Baseline) & 2nd Qtr.,FY14 \\
CD-3a (Approve Start of Long-lead Procurement) & 2nd Qtr.,FY14 \\
CD-3b (Approve Start of Construction) & 4th Qtr.,FY15 \\
Key Performance Parameters Satisfied & 4th Qtr.,FY19 \\
CD-4 (Includes 18 Months of Programmatic Float) & 2nd Qtr.,FY21 \\
\hline
\end{tabular}

CD (Critical Design), FY (Fiscal Year)

The way that Mu2e flows is that initially the experiment was approved based on the need to discover the muon to electron conversion. This stage was deemed CD-0 [1]. The next two stages, CD-1 and CD-2, delve into much of the design and performance work that needs to be done for Mu2e. CD-3 is broken up into two stages, CD-3a and CD-3b, which deals with the construction of the experiment. Finally, there will be tests done to confirm that the performance of the experiment is satisfactory and will be able to function after the construction stage. Once these parameters are met the experiment will be able to run. More information about the timeline for Mu2e can be seen in Table 1.1.

\subsection{Objective}

There is a short timeline to achieve engineering design, but also satisfy particle physics requirements. This thesis describes the design of the muon beam stop incorporating both its particle absorption performance and mechanical considerations. Chapter 2 will review previous work done by engineers and physicists at Fermilab, and will also define the constraints and goals of this thesis. Chapter 3 provides insight into G4Beamline, a particle physics simulation environment, which was used 
to find relationships between geometry, material, and particle absorption. Chapter 4 presents the results of the G4Beamline simulations, while Chapter 5 discusses the support structure that will be used for the MBS. Finally, Chapter 6 provides the conclusions from the research as well as open questions remaining for future research endeavors. 


\section{CHAPTER 2}

\section{BACKGROUND OF PRELIMINARY MUON BEAM STOP DESIGN}

The muon beam stop (MBS) was originally proposed to be a multi-material cylindrical component located within the detector solenoid. The purpose of the MBS is to capture remnant muons and shield the experiment's detection systems from the particles produced by the muons, mostly electrons, photons, and neutrons. This shielding is crucial for both particle tracking purposes and minimizing radiation damage to components such as photodetectors in the cosmic ray veto.

\subsection{Characteristics of the Muon Beam Stop}

The base design of the MBS is made of a main 316L stainless steel tube with polyethylene on the inside and lead on the outside near the back of the steel tube. The baseline design is fully defined in Mu2e-doc-1383, but the main qualities of this design will be highlighted. The conceptual design can be seen in Figure 2.1, with material types and subcomponents clearly identified by colors and callouts. It can be seen that there are several different acronyms used to describe these components.

The 316L stainless steel component is referred to as the BSTS and is used for electron and gamma absorption, while the lead component known as the SBPS is mainly used for gamma absorption because of its higher density. The polyethylene components are the BSBS, BSTC, and CLV2 and like the lead component it is used 
for neutron absorption. There are associated material properties with the three materials used to construct the MBS, and they can be seen in Table 2.1 [9].

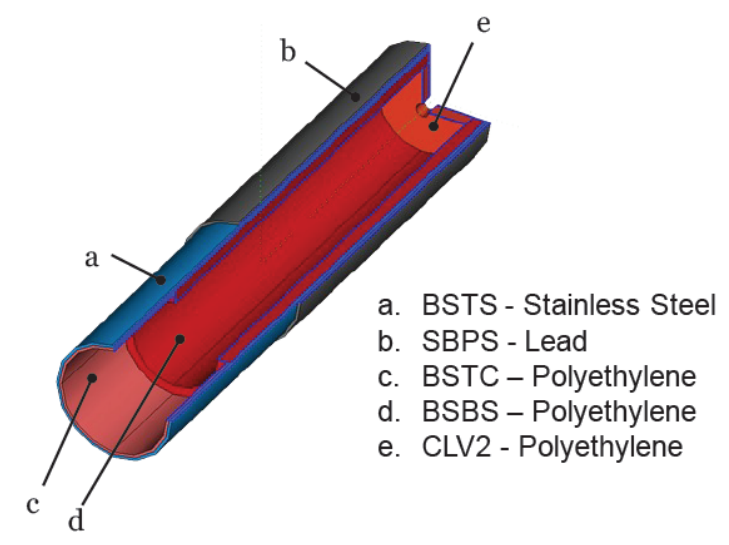

Figure 2.1: Components of the MBS [9]

Table 2.1: Material Properties of MBS Materials [9]

\begin{tabular}{ccccc}
\hline \hline Material & Density(g/cc) & UTS(MPa) & YS(MPa) & MOE(GPa) \\
\hline 316L SS & 8.04 & 565 & 248 & 193 \\
Lead & 11.37 & 16.5 & 11 & 13.8 \\
Polyethylene & $.918-.105$ & $10-43(31.1$ ave.) & $9-43$ (26 ave.) & $.18-1.57(.93$ ave.) \\
\hline UTS(Ultimate Tensile Strength), YS(Yield Strength), MOE(Modulus of Elasticity)
\end{tabular}

Table 2.2 identifies the dimensional scale of the current MBS design. The longest component is the BSTS which is just over 4 meters long. The MBS is this long because the magnetic field ends and particle have to move radially within the MBS in order to interact with MBS material. As well, the biggest component of the MBS in terms of outer diameter is the SPBS which sits at $920 \mathrm{~mm}$.

The positioning of the materials for the MBS is crucial as well because "hot spots" can occur within the MBS. Those areas with higher frequency of particle impact may require thicker layers of material to fully absorb the energy. Therefore, adding more material or the right kind of material in specific areas can help to 
Table 2.2: Dimensions and Mass of Current MBS Components [9]

\begin{tabular}{ccccc}
\hline \hline Component & ID $(\mathrm{mm})$ & OD $(\mathrm{mm})$ & Length $(\mathrm{mm})$ & Mass $(\mathrm{kg})$ \\
\hline BSTS & 920 & 858 & 4116 & 1789 \\
SPBS & 858 & 902 & 2640 & 1830 \\
BSTC & 773 & 815 & 650 & 40 \\
BSBS & 633 & 815 & 3366 & 751 \\
CLV2 & 101 & 815 & 100 & 33 \\
\hline
\end{tabular}

maximize particle absorption in the MBS while preventing the overall mass from increasing.

One problem with adding more material to the MBS is that adding material also adds weight, and ultimately the MBS has to be supported and installed into the experiment. The current MBS design weighs $4443 \mathrm{~kg}$ and is supported by three supports that sit on six bearing blocks [9]. As originally designed, the lead was not uniformly placed on the MBS resulting in an unbalanced system with a center of mass skewed toward the back end of the beam stop. Equation 2.1 shows that the calculation used to confirm the assumption of the COM being toward the back end of the MBS is in fact correct. The Z-direction is taken along the axis of the MBS.

$$
z_{C O M}=\frac{\sum_{i=1}^{n} m_{i} z_{i}}{M_{\text {Total }}}
$$

After plugging in the appropriate values from the tables for the individual masses, center of mass locations, and total mass, the center of mass of the MBS is calculated to be at about $2444 \mathrm{~mm}$ from the front of the MBS, which is in the back half of the MBS by around $386 \mathrm{~mm}$. Taking these facts into consideration, engineers at Fermilab have designed a support structure that will accommodate these restrictions of the very unbalanced load. Figure 2.2 shows the needs for double support structure as close to COM as possible. Also, the rails that the MBS support structure will 
sit on end almost coincidentally with the back of the support structure leaving approximately $1500 \mathrm{~mm}$ of the back of the MBS to be cantilevered.

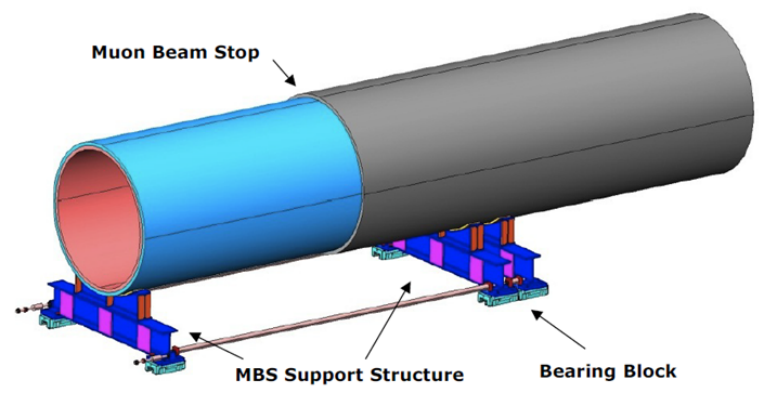

Figure 2.2: MBS Supported [9]

The support structure is essentially three weldment supports that contour the outer radius of the MBS. The weldment supports sit atop bearing blocks that will be on a rail system allowing the MBS to be installed inside the DS and precisely aligned with stopping target, tracker, and calorimeter. Other features that can be seen are the spacing rods that ensure the supports are spaced properly. There are six bearing blocks total, two for each support weldment, and four of them are toward the back end of the MBS where most of the weight is. These four bearing blocks will take the brunt of the force exerted by the MBS on the bearing blocks. Any reduction or redistribution of weight can reduce the loading of the bearing blocks on the rail system.

\subsection{Proposed Installation and Previous Analysis}

Assembly of the MBS and other components within the DS bore is a very crucial stage of the experiment. This is because there needs to be precise and accurate alignment of components within the DS in order for the experiment to run as smoothly as 
possible. Alignment is critical because of the stopping target, tracking, and shielding. All three of these aspects depend on being aligned properly inside the DS, with a requested accuracy around $+/-0.5 \mathrm{~mm}$ accuracy. For example, if the calorimeter or target tracker are not aligned properly they might return incorrect results to the experiment.

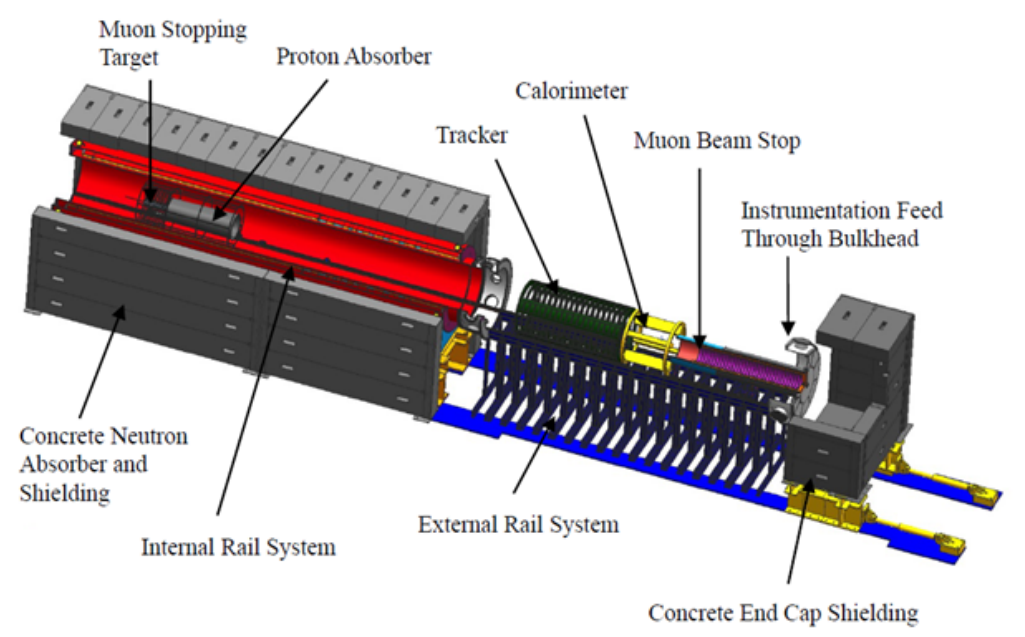

Figure 2.3: Detector Solenoid Components Installation [10]

Figure 2.3 depicts an exploded view of the DS which will be used to describe the intended installation process. The current installation plan is to utilize both an internal and external rail system for transporting components in and out of the DS. With the muon stopping target and proton absorber already installed, the target tracker, calorimeter, and MBS will be transported on external rails [10]. The instrumentation feed through bulkhead (IFB) and end cap concrete shielding will be transported on a separate set of rollers [10].

By utilizing an external and internal rail system, components will be able to move both inside and outside the DS. While components are inside the DS, they will be adjusted axially to the intended positions for the experiment, both individually and as an assembled train when all components are aligned. A section view of the 
DS with the components installed in the intended positions can be seen in Figure 2.4, although the cantilevered MBS depicted in the figure is not the MBS that will be used. The horizontal dimensions represent lengths of the different components within the DS. The vertical dimensions represent locations in the DS in terms of an absolute location where the center of the TS is the origin $(0,0,0)$. Regardless, these vertical dimensions can be used to find the intended spacing for components adjacent to one another in the DS.

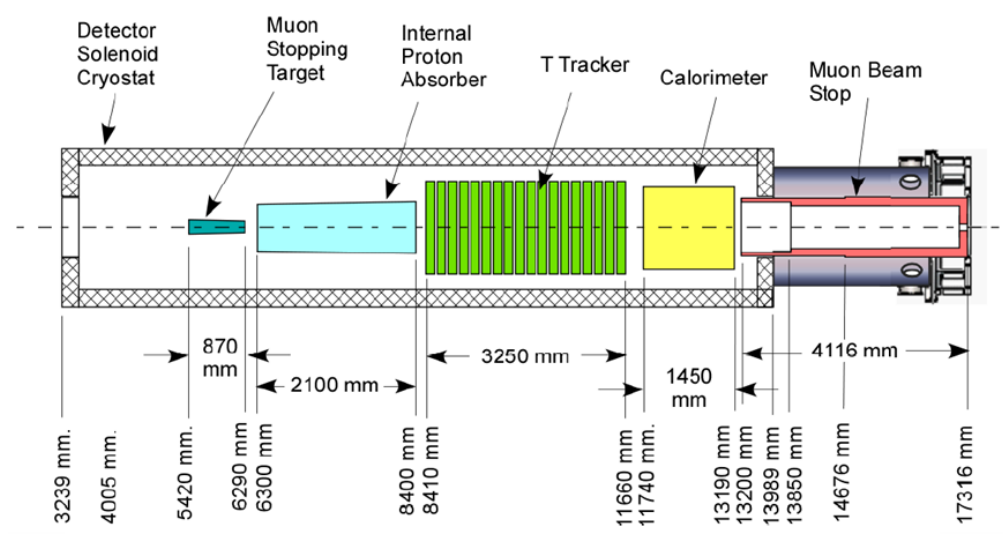

Figure 2.4: Section View of Assembled Detector Solenoid [10]

These internal and external rail systems must be confirmed that they will be able to withstand the stresses and deflections that will occur during and after installation. The rails will be attached to the inside walls of the DS as shown in Figure 2.5.

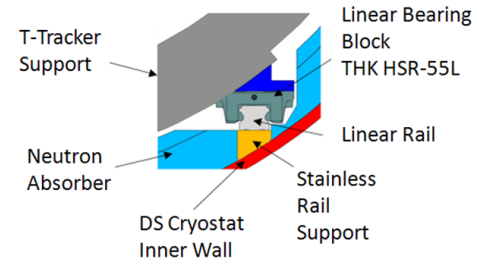

Figure 2.5: Internal Rail System [11]

Given that the MBS is the largest component in the DS, its weight distribution will set the overall size of the bearing blocks and corresponding rails for all other 
upstream components. Finite element analysis (FEA) can be used to model the forces that will be seen by the bearing blocks holding the MBS during and after installation. The model used takes into account the positioning of the MBS with respect to the rail system and the weight of the MBS. Figure 2.6 shows the analysis done and the results from a prior study in support of CD-0. The analysis utilizes the fact that the MBS is symmetric which allows the analysis to be a half model.

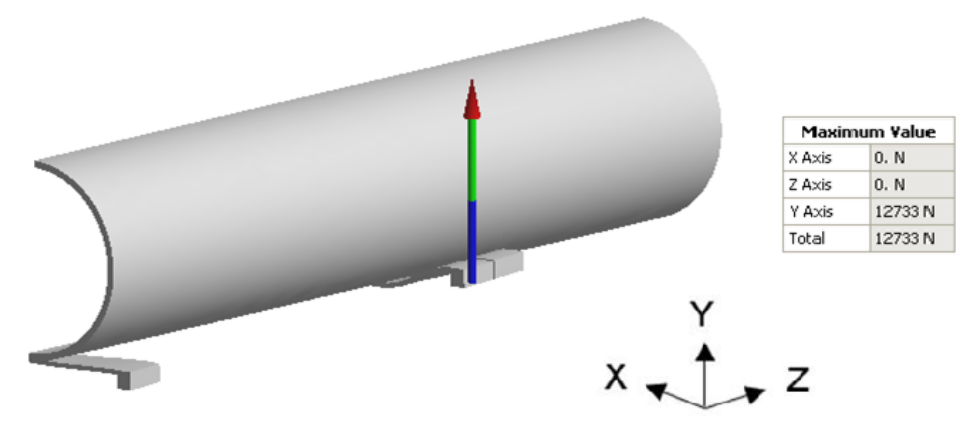

Figure 2.6: FEA for Internal Rail Bearing Blocks [11]

This shows that the maximum reaction force that will be seen by an individual bearing block is approximately $12.7 \mathrm{kN}$ in the Y-direction [11]. Similarly, THK, a linear motion guide company, ran a similar analysis of the MBS and bearing blocks yielded a reaction for of $12.3 \mathrm{kN}$ in the Y-direction [11]. With this analysis, Table 2.3 was utilized in order to properly select a bearing block that will withstand the reaction forces seen.

Table 2.3: THK Bearing Block Load Ratings (N) [11]

\begin{tabular}{lcccccc}
\hline \hline & HSR45 & HSR45L & HSR55 & HSR55L & HSR65 & HSR65L \\
\hline$C_{0}$ (Standard) & 60000 & 80400 & 88500 & 119000 & 141000 & 192000 \\
C (Standard) & 95600 & 127000 & 137000 & 183000 & 215000 & 286000 \\
$C_{0}$ (HPM75) & 16800 & 22512 & 24780 & 33320 & 39480 & 53760 \\
C (HPM75) & 17208 & 22860 & 24660 & $\mathbf{3 2 9 4 0}$ & 38700 & 51480 \\
\hline \multicolumn{7}{c}{$C_{0}$ (Static Load), C (Dynamic Load), HPM75 Stainless Steel }
\end{tabular}


Based upon the analysis done, load ratings of the bearing blocks, and maintaining a factor of safety greater than 2.5, the HSR55L-HPM75 bearing blocks were selected. Using Fermilab's reaction force of $12.7 \mathrm{kN}$ yields a factor of safety of 2.58 , while using THK's value yields a factor of safety of 2.67 [11]. Regardless of the value used, the factor of safety satisfies the constraint of being at least 2.5. Similar analysis was done to confirm the stresses and deflections that will occur on the external rail system. The results yielded a factor of safety above 2 and deflections well below $1 \mathrm{~mm}[11]$.
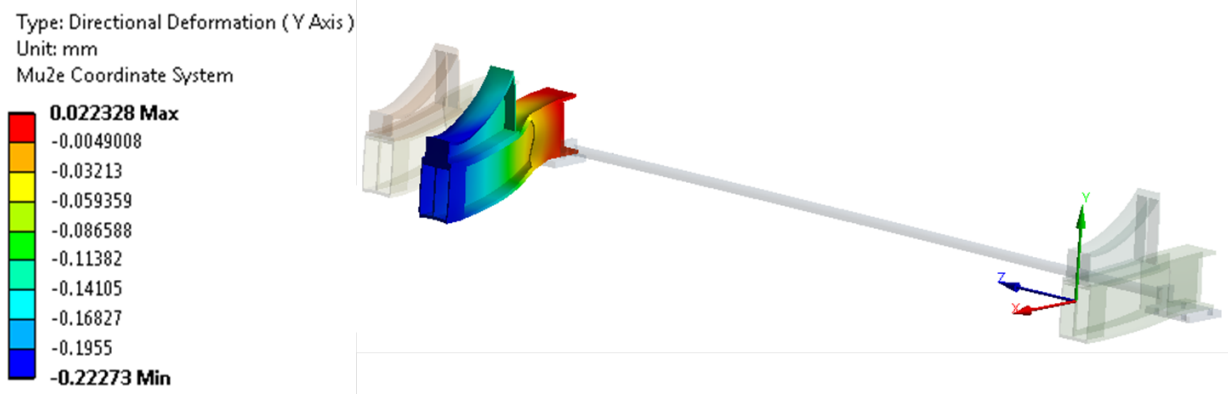

Figure 2.7: Deflection Along the Y-axis of the MBS Support Structure [12]

As well as the rail system, the support structure must be analyzed from a stress and deflection standpoint. The three support structures are made primarily from W6X16 I-beam, but also has plate steel that is used for stiffening the structure [12]. The support structure was simulated by using the reaction forces that the MBS will put on the structure. The structure was also simulated under a dynamic situation. The only dynamics will be the slight vibration as the MBS is rolled into the DS during installation. The goals of these simulations were to ensure that the total deflection of the support structure was less than $2 \mathrm{~mm}$ in any directions and the maximum stress is small enough to provide a factor of safety of at least 2 [12]. The analysis shows that the support structure meets these requirements for the current 
MBS. Figure 2.7 show an example of the simulated deflection that the MBS will see in the Y-direction.

All of the previous analysis confirms that the current MBS and support structure meets the requirements of the experiment. The support structure will be able to handle the MBS under both static and dynamic cases during installation and throughout the duration of the experiment. The MBS is able to provide a means of shielding for the experiment, but the question is whether or not there are better configurations for the MBS and its support structure.

\subsection{Goals and Constraints}

Moving forward, further exploration needs to go into the MBS design from a particle absorption perspective. There are several areas which can be considered in order to improve particle absorption such as material and size. As well, changing these parameters have an impact on the support structure used to hold the MBS up. The analysis will be an iterative process, in which the particle physics simulations will be run and analyzed and then structural analysis will be done to determine if the geometry is a plausible candidate for a new MBS. Keeping the ultimate goal of finding an MBS that improves particle absorption but does not sacrifice structural integrity in mind, there are also several constraints.

From a particle absorption stance there are certain types of particles that are sought out to minimize. One of the types of particles are neutrons exiting the sides of the MBS. These neutrons can penetrate the calorimeter and concrete shielding and then interact with the plastic that makes up the cosmic ray veto counters. Therefore they need to be absorbed, as much as possible, by the MBS. The other 
two types of particles are electrons and gammas greater than $100 \mathrm{keV}$ exiting the front of the MBS. These particle have potential to create problems with tracking particles in the target tracker and calorimeter.

Another constraint is the limitation of space. Highlighted in Figure 2.8 is the possible extra space in the DS for the MBS. The solid blue line shows the current MBS, while the dashed red line indicates possible extra space within the DS for the MBS. Some space is still necessary for cables to transfer data from target tracker and calorimeter, but Fig 2.8 shows what should be available in the cryostat.

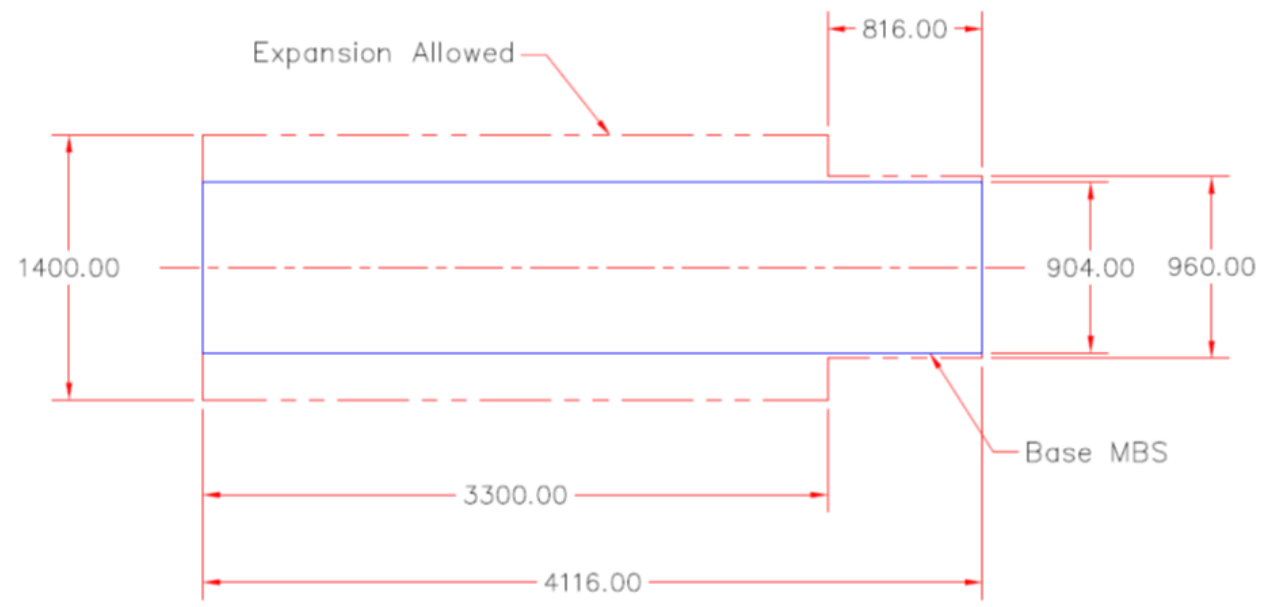

Figure 2.8: MBS Expandable Space

Now that the particle physics and spacing constraints have been defined, the structural support constraints must be stated. Similar to the previous structural analysis the constraints are to maintain less than a $2 \mathrm{~mm}$ total deflection as well as a factor of safety 2.5 or above. With these constraints defined, that leaves three different options to support the MBS.

The first option is to have a one piece MBS that is solely supported by MBS supports. If this option was chosen, it would require that the center of mass to be at least $3000 \mathrm{~mm}$ from the back of the MBS and that the mass cannot exceed 
$4920 \mathrm{~kg}$ [13]. The second option would allow the MBS to be split into two pieces which would be supported by both MBS supports and off the IFB. The limitations of this option are that the center of mass must be at least $2500 \mathrm{~mm}$ from the back of the MBS, and the mass must be lower than $4480 \mathrm{~kg}$. The third option is to have a one piece MBS that is supported off of both MBS supports and the IFB. The constraints are the same as the second option except the allow a $+/-10 \%$ deviation in both the center of mass and maximum mass [13].

With these fully defined constraints and particle physics goals, there are also engineering goals that expand further than maintaining the structural integrity of the support structure. One of these is a better positioned center of mass because this would provide a better loading distribution for the bearing blocks and rails. Likewise, utilizing the IFB as a support is not out of the question as this would help remove some of the load off of the rails and onto the IFB. If some of these options could be utilized, a smaller sized bearing block can be used without loss of factor of safety. Additionally, a new MBS geometry improves absorption with corresponding influence on the physics requirements for negligible backscatter and neutron penetration. The end result of the new MBS would be much more favorable than the current MBS and support structure. 


\section{CHAPTER 3}

\section{SIMULATIONS IN G4BEAMLINE ENVIRONMENT}

G4Beamline is a particle physics simulation environment used at Fermilab National Accelerator Laboratory to evaluate how changing components in the experiment will effect the particle physics [15]. G4Beamline is a set of code developed by Tom Roberts of Muons, Inc to run geant4 [16]. This software allows the user to vary parameters such as geometry to see how outcomes such as particle absorption will be affected. In order to analyze the MBS, G4Beamline was used to simulate particle absorption of the different MBS geometries that consider dimensional and material variation.

\subsection{Accessing G4Beamline}

All the files needed to run a G4Beamline simulation are saved in one's home directory on detsim.fnal.gov. Detsim.fnal.gov is a linux machine where G4Beamline simulations can be run. G4Beamline is setup to run off Linux machines, but it can also be run off a PC through terminal programs like Cygwin and its GUI interface XWin that are used to access detsim.fnal.gov.

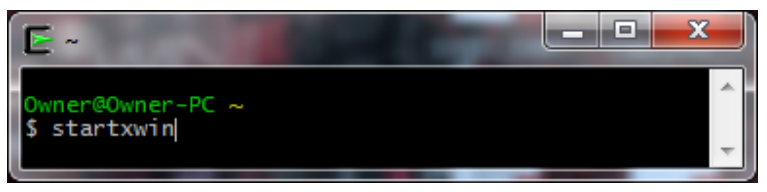

Figure 3.1: Accessing XWin Through the Cygwin Terminal 
Figure 3.1 shows how to start XWin from Cygwin. Also, Figure 3.2 shows how to login to Fermilab computing and begin accessing detsim.fnal.gov. The first step to logging in is to execute the kinit command which obtains the Kerberos ticket. The Kerberos ticket allows the computer to communicate with the Fermilab nodes. After the kinit command is executed it will prompt for the Kerberos password. Once the password is entered, one can connect to detsim.fnal.gov, which can be executed using the third line of executables in Figure 3.2.

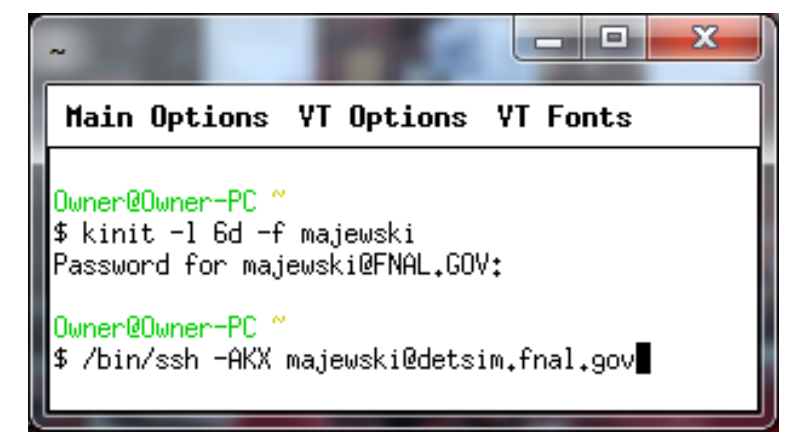

Figure 3.2: Accessing detsim.fnal.gov Through Xwin

While on detsim.fnal.gov, all the files needed to run a G4Beamline simulation are accessible as well as the Root files created after every simulation. Sorting the data in Root files will be used later in the post-processing. The other crucial files that are within the detsim user's home directory are the input (*.in) and geometry files (*.txt). All of these files can be found within G4BeamlineScripts/, which is a subdirectory underneath the user's home directory. Figure 3.3 shows the standard files within the user's home directory inside detsim. As well as the Root, input, and geometry files there are also setup and macro file which will be used for setting up and post processing, respectively. The figure also shows the commands executed in order to access these files and directories once logged in to detsim.

Some of the common commands that are used include cd and ls, which stand for change directory and list, respectively. A very helpful source for Linux commands is 


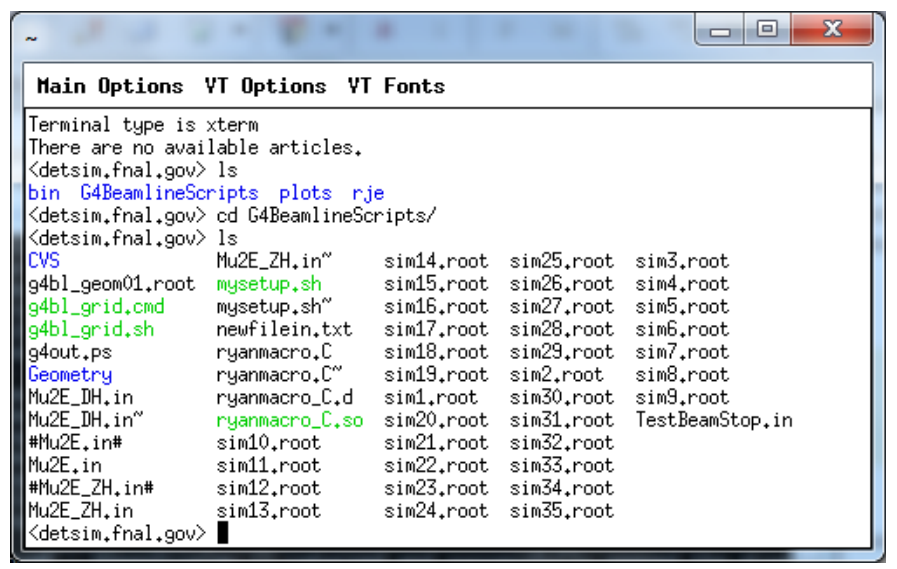

Figure 3.3: Detsim User Home Directory

http://www.pixelbeat.org/cmdline.html. For this thesis, the only file that will be changed from simulation to simulation is the geometry file, DS_Muon_Beam_Dump.txt which is under the Geometry directory. The rest of the files, including the input file, remain constant.

\subsection{Input File}

The input file used for G4Beamline contains various items that sets up the parameters of the simulation. One of the first parameters that can be changed in the input file is the number of events (or total number of muons) considered in a single simulation run. In all studies presented below, 300,000 muons with the same initial conditions are considered. Additionally, the different colors and materials that are available to use are set up in the input file. Using the correct materials is a crucial aspect of running a simulation because all the materials have different densities, and materials with different densities will impact absorption rates. These materials can be defined in the input file by the different elements that they are composed of, which is included in Appendix B on page 57. 
Outside of the basic applications mentioned above, the input file also calls in all the geometries used in the simulations. The DS_Muon_Beam_Dump.txt file contains the MBS geometry used in simulations. Likewise, there are geometry files for all the components in the experiment such as the tracker, cryostat, and concrete blocks that house the experimental solenoids. Other important feature are that the input file sets a coordinate system at the center of the TS, and sets up the particle tracking. The coordinate system is crucial for geometry placement as well as post processing. For particle tracking, identification numbers called PDGids are assigned to the various particles. Likewise, the colors of the particle can be changed in the input file, so each particle's track can be distinguished as a visual aid in post processing.

Finally, one of the most important aspects of the input file is that it allows the user to input virtual detectors to the simulation. A virtual detector acts as a massless plane that can be placed anywhere within the simulation in order to count on particles passing through certain regions. The virtual detectors will not effect the outcome of the experiment if placed in open space, but it could potential have an impact if it interfering with other geometry within the simulation. The input file used for the simulation ran is provided in Appendix B. The commands creating and placing the virtual detectors can be seen toward the end of the input file. The positions of the virtual detectors are at the front of the MBS, around the MBS, and at the back of the MBS. Sometimes virtual detectors at the back of both the Target Tracker and Calorimeter were used for evaluation of the back scatter in the MBS. 


\subsection{Geometry Files}

A typical geometry file for the last simulation run, with the final MBS dimensions, can be seen in Appendix C. The file includes all the various pieces that compose the MBS and each component is defined geometrically, by material type, and placed in the simulation environment. The process for defining a component within the simulation starts by describing its dimensions and material. For this case, the cylinder's geometry is defined by an outer radius, inner radius, and length. Once these are defined the next step is to place the geometry in the simulation space. Remembering that the placement depends on the absolute coordinate system where the middle of the TS is the origin, the geometry can be appropriately placed. Upon defining these parameters, the geometry can be successfully placed in the simulation once the input file calls for this geometry file.

Other than defining and placing the object, the geometry file also defines other parameters. Some of the other definitions within the geometry file are colors and visibility. By defining colors to geometries the placement and dimensions can be confirmed to an extent later in post processing. Likewise, visibility allows the user to define a cylindrical feature to be partially exposed. This can be done by defining two cylindrical tubes with the same geometry but different angles and colors. For example, the first tube can be defined to be blue and range from $(\pi / 2,0)$, while the second tube can be defined to be invisible and range from $(0, \pi / 2)$. Both of these cylinders have the same geometry, material, and placement, therefore they combine to make one cylinder where the user can see inside the cylinder from the range $(0, \pi / 2)$. The visibility options are also helpful during post processing because they can be used to make sure that the correct geometry has been defined in the proper 
place. Another benefit from make these cylinder's invisible for a quadrant or so is that the particle tracks can be seen in post processing.

\subsection{Running a Simulation}

After logging into detsim and configuring the input and geometry files to ones liking, there is still some setup to be done before running a simulation. There are three things that still need to be configured which include the Mu2e setup, Root setup, and G4Beamline setup. The Mu2e setup allows the user to gain access to all the Mu2e software on the cluster which is important because it allows the user access to run a G4Beamline simulation [17]. The Root setup is needed in order to make plots or take particle counts in post processing. Finally, the G4Beamline setup accesses the version of G4Beamline that the user wants to use, as G4Beamline is updated from time to time, for there simulations. The commands for these can be executed individually every time one logs into detsim, or a setup file can be written that will take care of all three at once. With the help of Zachary Hodge, a setup file was created and can be seen in Appendix D, similarly Figure 3.4 shows how to source the setup file.

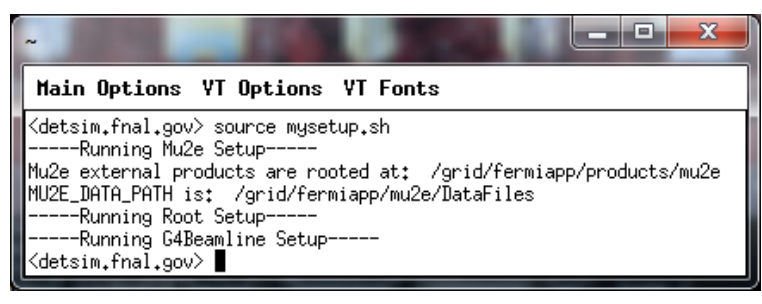

Figure 3.4: Command to Source the Setup File

The first line in the figure is the command used to source the setup file. Once this command is executed, the file will setup everything needed to run a simulation and 
extract data from a simulation. While the program is running, it will tell you each setup that it is running, which can be seen in the figure. Once setup is complete, the command line for detsim will re-appear, and a simulation can now be run. Figure 3.5 shows the command that will start the simulation.

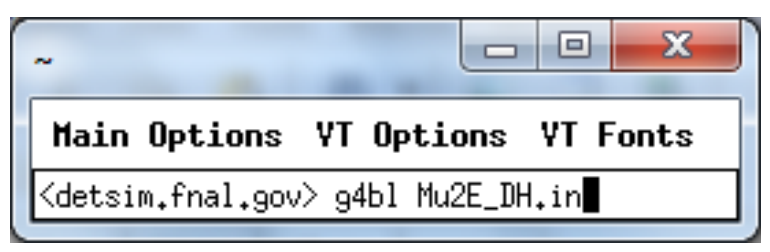

Figure 3.5: Running a G4Beamline Simulation

This command follows the syntax $\mathbf{g} \mathbf{4 b l}<$ inputfile $>$. Therefore, one can have multiple input files that can be used for different simulations. Upon completion of a simulation, an output will be displayed conveying the main components of the simulation such as how many events were used and how many events per second were run. Also, the number of entries per virtual detector will be displayed. Finally, every time a simulation is run a Root file will be created in the user's home directory. This Root file will always be named $\mathbf{g} 4$ beamline.root, and should be renamed after each simulation. If it is not renamed, the next time a simulation is run the file will be written over with the new simulations Root file. Figure 3.6 shows most of the output shown on the XWin window after a simulation is run.

It can be seen that for this simulation, ten events were run. In addition to the counts that are displayed on the XWin window, there is another, more visually appealing, way to run a G4Beamline simulation. By using event display, one will be able to actually view the geometry and particle tracks in the simulation. In order to enable this viewer, instead of typing the command $\mathrm{g} 4 \mathbf{b l}<$ inputfile $>$ one must type $\mathbf{g} 4 \mathbf{b l}<$ inputfile $>$ viewer $=$ best [17]. Once this is done the simulation will 


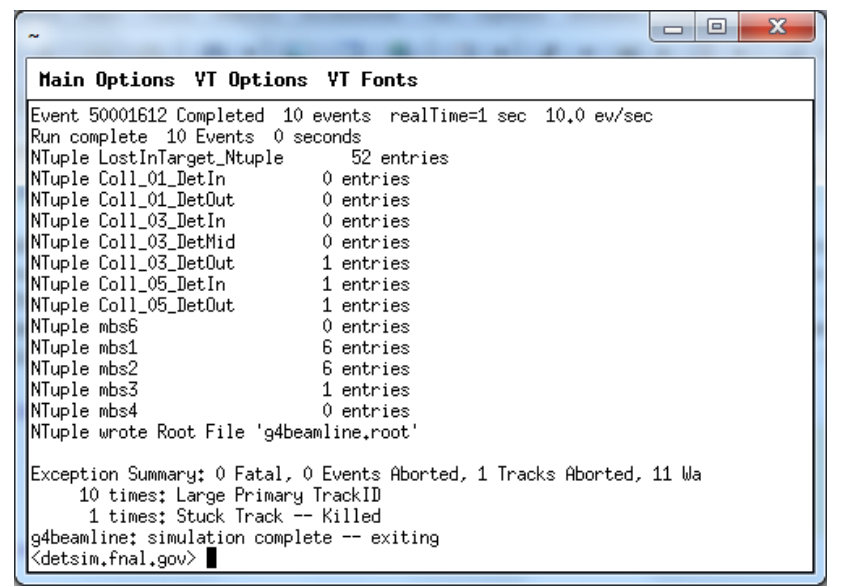

Figure 3.6: Outputs to XWin Window

set up and run and then prompt the user to initiate the viewer. This can be seen in Figure 3.7.

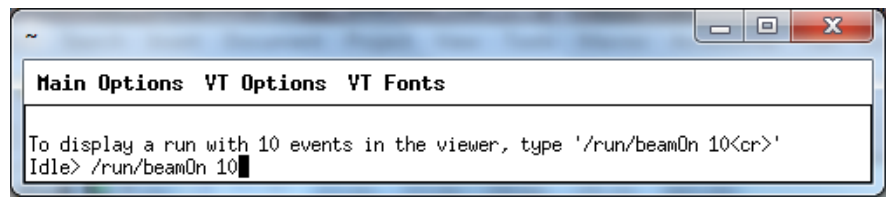

Figure 3.7: Command to Start the Viewer in G4Beamline

Once executed, the viewer will start and the user will be able to manipulate the orientation of the geometry in the viewer. Additionally, the particle tracks can be seen and are the green and red tracks in Figure 3.8. With the particle tracks, the MBS can be seen toward the left side of the figure in red, blue, and yellow. This figure also represents the benefit of using the visibility options when defining geometry.

Only ten muons were used for the simulation where the viewer was used because running a simulation with the viewer on would take longer than with it off. On average a simulation that was run with 300,000 muons as the input took around 


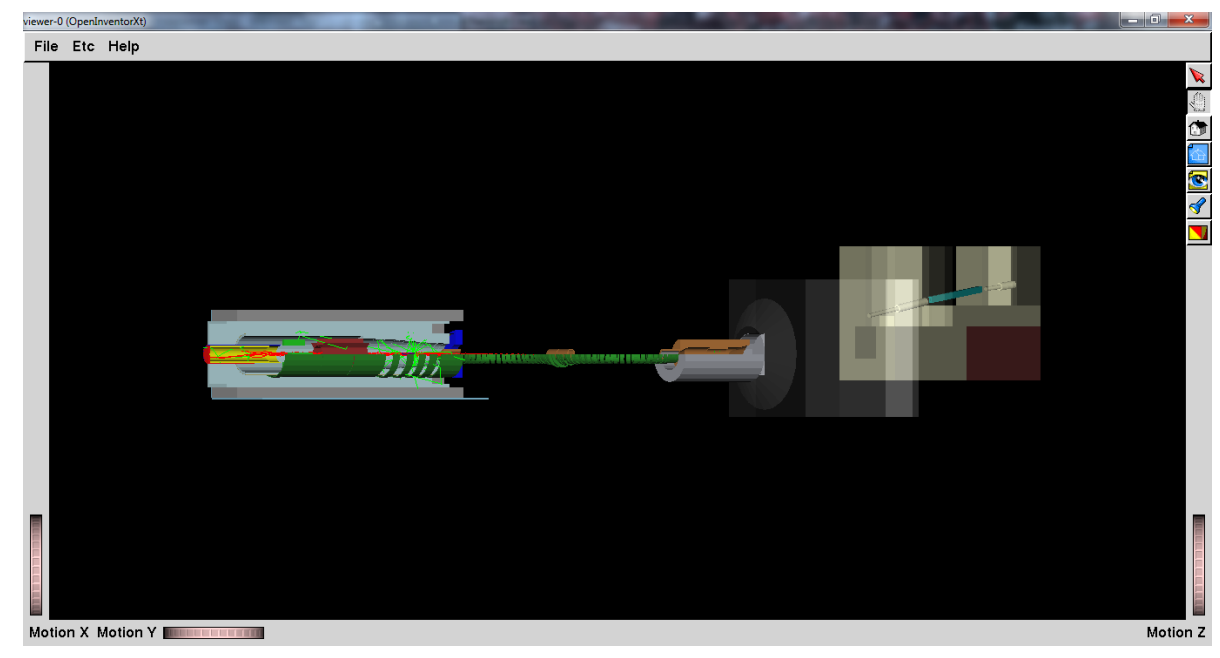

Figure 3.8: G4Beamline Viewer

four to five hours on average to complete, and this was never tried with the viewer on.

It should be stated that there is error within the simulations. Both statistical and systematic error occurs during the simulation. Statistical error roots from the fact that each time a simulation is run, G4Beamline starts with a different random number. While for this case, the biggest systematic error of concern lies within the neutrons. This error is dependant upon how many neutrons are made and what the energies of the neutrons are when a muon is captured on a different nuclei [18]. The fluctuation and minimal percent changes for the gammas and electrons spawn from these errors. All of the data within the simulations are stochastic data and not a deterministic solution.

\subsection{Post-Processing}

Once the simulation is done running, a Root file will be written to the user's home directory. Like previously mentioned, this Root file should be renamed so it 
will not be written over when the next simulation is ran. Configuration and control of simulation parameters is important for tracking performance changes as a result of variable modifications. After this, the Root file can be used to extract data from the simulation. In order to extract data from the Root file, a macro was written with the help of Zachary Hodge.

The macro, which can be seen in Appendix E, reads information from the Root file. It is programmed to read a Root file from the user's home directory. The user can change which Root file is to be read inside the macro. Once the user decides what Root file is to be used, the macro can be executed using the following commands seen in Figure 3.9.

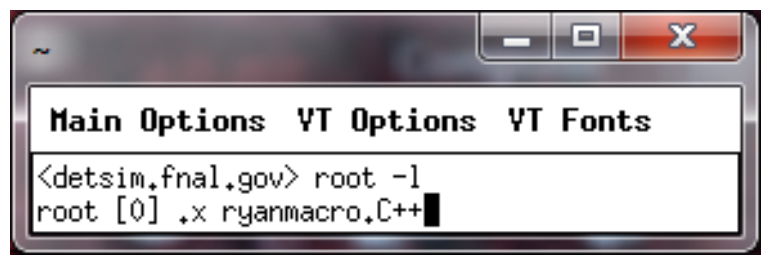

Figure 3.9: Starting Root and Macro

The first line starts Root while the second line executes the macro. The macro will create 4 plots of total particles, neutrons, electrons, and gammas $(100 \mathrm{keV}+)$ that cross a selected virtual detector. The virtual detector can be selected within the macro before executing the macro. These plots are created by using the particles PDGid's and the particle's momentum. For the virtual detectors in front of the MBS one has to use negative momentum as a criteria because the concern is particles coming back through the front of the MBS. While for the virtual detector at the back of the MBS a condition can be made to only take particles with positive momentum. Likewise, a condition for particles exiting the virtual detector surrounding the MBS can be made. Taking all these conditions and options into account, once the macro is run a density plots will be made for the selected conditions and virtual detectors. 
Figure 3.10 an example of what one of these plots look like. Finally, the last thing the macro does is save the four plots to a plot folder within the user's home directory.

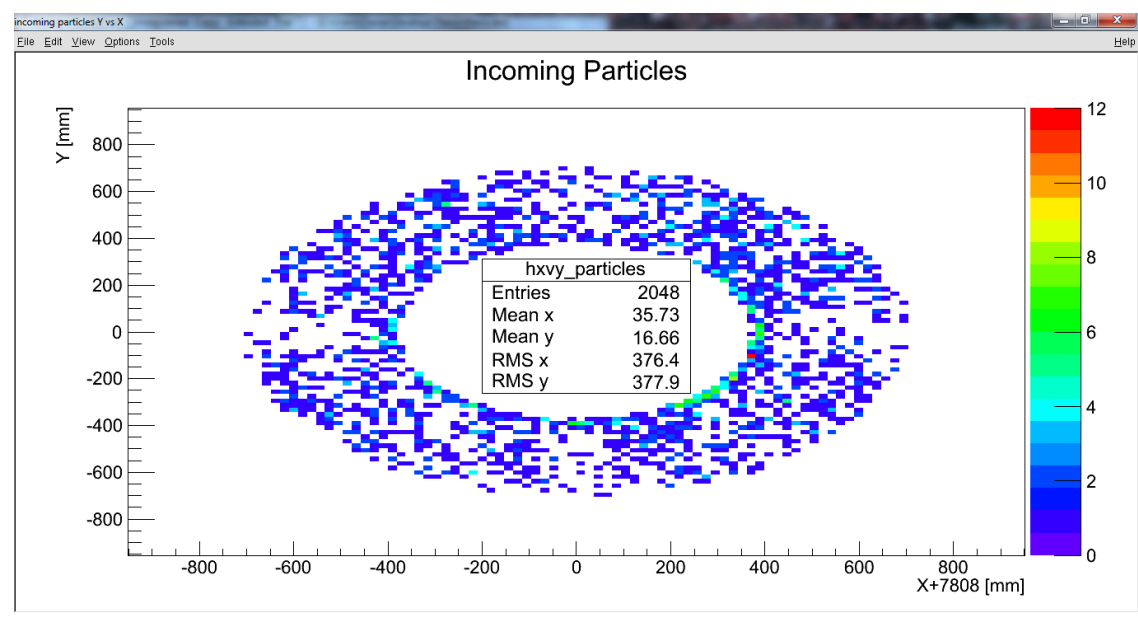

Figure 3.10: Example Output from the Macro

In the selected plot above, the density of particles entering the MBS through a selected virtual detector can be seen. The bottom scale shows the $\mathrm{X}$-axis in millimeters and the left scale shows the Y-axis in millimeters, note that the two dimensions are not scaled uniformly. The MBS is indeed circular in shape. On the right is a density scale that represents the number of particles which can be used to gage the locations of high and low particle occurrence. Likewise, above the plot there is a label that shows what type of particle are being plotted, and in this case the plot shows any type of particle that crosses this particular virtual detector while headed into the MBS. Finally, there is also a box in the middle of the plot that shows how many of particles crossed the selected virtual detector, the average $\mathrm{X}$-position, average $\mathrm{Y}$-position of the particles, and the root mean square of the $\mathrm{X}$ and Y positions. Now with the ability to run a simulation and adjust geometries to examine the back scatter of gammas $(100 \mathrm{keV}+)$ and electrons and radial neutron radiation, iterations can be run for the MBS in order to meet physics requirements. 


\section{CHAPTER 4 G4BEAMLINE SIMULATION RESULTS}

Six different G4Beamline simulation sets were run to see how the particle physics was effected by geometric changes. The first three simulation sets were intended to evaluate what the relevance of the lead was in terms of shielding. The last three simulation sets were intended to optimize the MBS internal and external dimensions and materials, taking into account the results of the first three simulation sets. Continued iterations were conducted after these results were reported. The final simulation evaluation concludes this chapter and then is evaluated for structural integrity in Chapter 5.

\subsection{Geometry Study 1}

The first geometry study was set out to explore the usefulness of the lead component (SPBS) at the back end of the MBS. All of the other components of the MBS were held constant, while the SPBS was changed radially. The length of the SPBS was the same $2640 \mathrm{~mm}$ length and same axial position. The radius was incremented out to the $480 \mathrm{~mm}$ maximum radius that was given by the space available to expand defined by the back end of the MBS, which shown in Figure 2.8 in Chapter 2. Table 4.1 reveals the results in terms of particle physics and geometric change.

The bold results are the current MBS dimensions and they will be used as a reference for all other study results moving forward. Also, the results are shown 
Table 4.1: Study 1 Results

\begin{tabular}{cccc}
\hline \hline Pb Radius $(\mathrm{mm})$ & \# n Exit Sides & \# g (100kev+) Exit Front & \# e Exit Front \\
\hline $\mathbf{4 5 2}$ & $\mathbf{1 3 5 8}$ & $\mathbf{2 8 4 9}$ & $\mathbf{3 1 5 5}$ \\
460 & 1318 & 2862 & 3138 \\
470 & 1289 & 2799 & 3151 \\
480 & 1241 & 2798 & 3136 \\
\hline
\end{tabular}

Lead (Pb), Neutrons (n), Gammas (g), Electrons (e)

in terms of number of particles that cross the indicated virtual detector. It can be seen that the number of neutrons exiting the sides decrease by $8.6 \%$ as the radius of the lead at the back end is increased. Table 4.2 show all the percentage changes of the first simulation. The table shows that the biggest particle impacted was the neutrons exiting the sides of the MBS, while the electrons and gammas were minimally affected. The neutrons exiting the sides of the MBS decrease as the radius of the lead increases because this change provides more of a higher density material to absorb these neutrons.

\begin{tabular}{cccc}
\multicolumn{4}{c}{ Table 4.2: Study 1 Percentage Change } \\
\hline \hline Pb Radius(mm) & \% Change n & \% Change g (100kev+) & \% Change e \\
\hline 460 & $-2.9 \%$ & $+0.5 \%$ & $-0.5 \%$ \\
470 & $-5.1 \%$ & $-1.8 \%$ & $-0.1 \%$ \\
480 & $-8.6 \%$ & $-1.8 \%$ & $-0.6 \%$ \\
\hline \multicolumn{4}{r}{ Lead (Pb), Neutrons (n), Gammas (g), Electrons (e) }
\end{tabular}

\subsection{Geometry Study 2}

The second geometry study removes the lead piece at the back end of the MBS, and increments the stainless steel tube radially (BSTS) to see the impact on the particle physics. Since the stainless steel tube spans the full $4116 \mathrm{~mm}$ length of 
the MBS, the radius is constrained by the back end expandable space. Therefore, the BSTS can only be incremented out to a radius of $480 \mathrm{~mm}$. Table 4.3 show the results in terms of number of particles exiting various parts of the MBS.

Table 4.3: Study 2 Results

\begin{tabular}{cccc}
\hline \hline SS Radius $(\mathrm{mm})$ & \# n Exit Sides & \# g (100kev+) Exit Front & \# e Exit Front \\
\hline 429 & 1480 & 3312 & 3141 \\
440 & 1225 & 2762 & 3154 \\
450 & 1114 & 2542 & 3168 \\
460 & 1036 & 2358 & 3147 \\
470 & 974 & 2271 & 3149 \\
480 & 897 & 2157 & 3147
\end{tabular}

Stainless Steel (SS), Neutrons (n), Gammas (g), Electrons (e)

Some relations that can be seen from the table are that the gammas (100kev+) exiting the front of the MBS and the neutrons exiting the sides of the MBS decrease as the BSTS radius is increased. The electrons exiting the front of the MBS stay almost constant while the BSTS radius is increased, and the minor fluctuations are due to error within the simulations. Table 4.4 displays the percentage change of each particle exiting their respective sections of the MBS compared to the baseline MBS geometry.

Table 4.4: Study 2 Percentage Change

\begin{tabular}{cccc}
\hline \hline SS Radius $(\mathrm{mm})$ & \% Change $\mathrm{n}$ & \% Change g (100kev+) & \# Change e \\
\hline 429 & $+9.0 \%$ & $+16.3 \%$ & $-0.4 \%$ \\
440 & $-9.8 \%$ & $-3.1 \%$ & $-0.03 \%$ \\
450 & $-18.0 \%$ & $-10.8 \%$ & $+0.4 \%$ \\
460 & $-24.0 \%$ & $-17.2 \%$ & $-0.2 \%$ \\
470 & $-28.3 \%$ & $-20.3 \%$ & $-0.2 \%$ \\
480 & $-34.0 \%$ & $-24.3 \%$ & $-0.3 \%$ \\
\hline
\end{tabular}

Stainless Steel (SS), Neutrons (n), Gammas (g), Electrons (e)

The largest percent change occurs in the neutrons exiting the sides of the MBS, which is a $34.0 \%$ decrease. Likewise, the gammas (100kev+) exiting the front of the 
MBS has a large percentage decrease, while there is roughly no change in electrons exiting the front of the MBS. It can be concluded that increasing the stainless steel in the MBS has the most effect on the neutron and gamma absorption. This is due to the fact that the stainless steel has higher density than the polyethylene and more of it is being added as the radius is increased.

\subsection{Geometry Study 3}

The third geometry study explores what the impact of moving the SPBS to the front of the MBS instead of having it at the back end of the MBS. The magnetic field has changed since the original design was considered which calls for an investigation of the hot spot at the axial location where the particles impact the MBS. The length of the SPBS is kept the same, and the only parameters that are changed are the position and the radius of the SPBS. Once again, the SPBS is $2640 \mathrm{~mm}$ and the radial expansion is limited by the back end constraint of a radius equivalent to $480 \mathrm{~mm}$. The position of the front of the SPBS is coincident with the front of the MBS. Table 4.5 shows the results in terms of number of particle exiting the MBS in various locations.

Table 4.5: Study 3 Results

\begin{tabular}{cccc}
\hline \hline Pb Radius $(\mathrm{mm})$ & \# n Exit Sides & \# g (100kev+) Exit Front & \# e Exit Front \\
\hline 452 & 1424 & 2140 & 3147 \\
460 & 1394 & 2091 & 3139 \\
470 & 1398 & 2028 & 3131 \\
480 & 1376 & 2011 & 3157 \\
\hline
\end{tabular}

Lead (Pb), Neutrons (n), Gammas (g), Electrons (e) 
From Table 4.5 and Table 4.6 it can be seen that the most affected particle from moving the lead component to the front end of the MBS is the gammas $(100 \mathrm{kev}+)$ exiting the front of the MBS. This is due to the fact that the higher density lead has been moved to the front of the MBS and increased, which allows for more absorption of the high energy gammas. These gammas experienced a decrease between 24.9$29.4 \%$ in comparison with the baseline geometry depending on the radius of the lead component. The electrons exiting the front of the MBS and the neutrons exiting the sides of the MBS experienced small change. The electron fluctuation was the smallest change and can be mostly attributed to simulation error. Neutrons exiting the sides were a slight increase in comparison with the baseline geometry.

\begin{tabular}{cccc}
\multicolumn{4}{c}{ Table 4.6: Study 3 Percentage Change } \\
\hline \hline Pb Radius(mm) & \% Change n & \% Change g (100kev+) & \% Change e \\
\hline 452 & $+4.9 \%$ & $-24.9 \%$ & $-0.3 \%$ \\
460 & $+2.7 \%$ & $-26.6 \%$ & $-0.5 \%$ \\
470 & $+2.9 \%$ & $-28.8 \%$ & $-0.7 \%$ \\
480 & $+1.3 \%$ & $-29.4 \%$ & $+0.06 \%$ \\
\hline \multicolumn{4}{r}{ Lead $(\mathrm{Pb})$, Neutrons (n), Gammas (g), Electrons (e) }
\end{tabular}

\subsection{Geometry Studies 4, 5, and 6}

Geometry studies 4, 5, and 6 explore a stepped down version of the BSTS, and filling the rest of the expandable space, similarly to the expandable space in Figure 2.8, with polyethylene. The only differences between the expandable space utilized in these studies and the expandable space shown in Figure 2.8 are that the front $3399 \mathrm{~mm}$ were limited to a maximum radius of $525 \mathrm{~mm}$ and the back $717 \mathrm{~mm}$ were limited to a maximum radius of $480 \mathrm{~mm}$. This difference is due to the fact that the 
finalized expandable space was in the process of being defined at the time that these simulations were running. Figure 4.1 shows a section view of the geometry used for the studies.

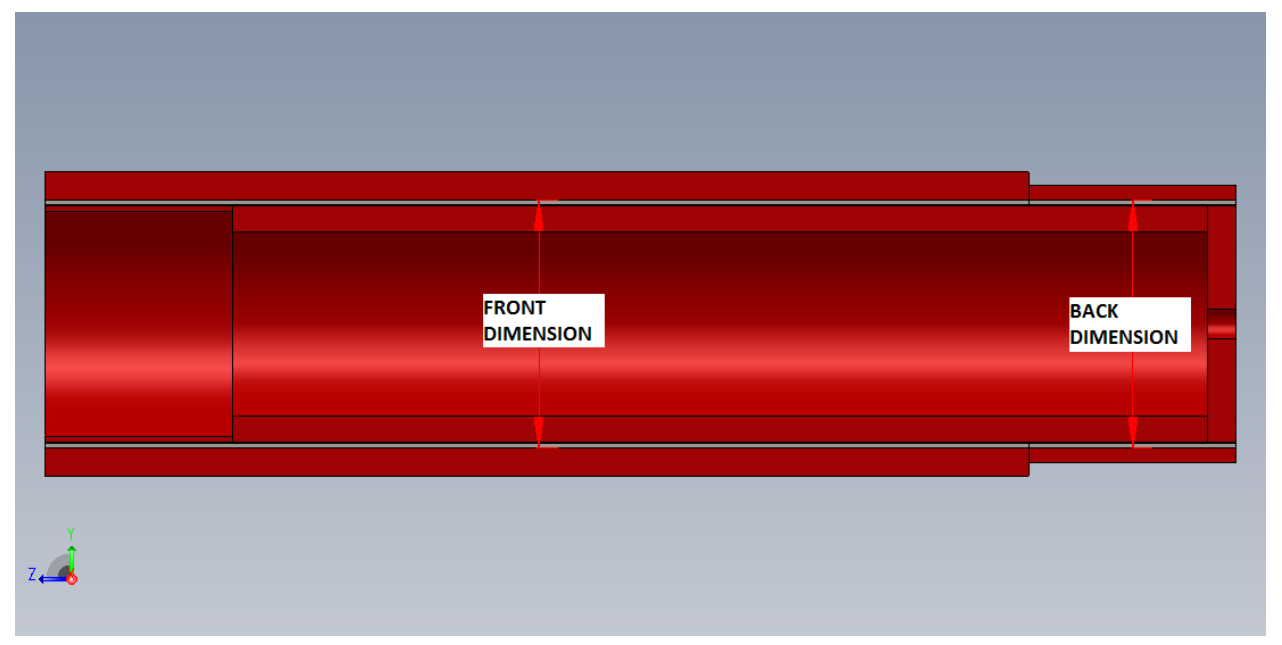

Figure 4.1: Geometry Definition for Studies 4, 5, and 6

Study 4 keeps the back radius constant at $429 \mathrm{~mm}$ while incrementing the front radius from $429 \mathrm{~mm}$ to $510 \mathrm{~mm}$ in increments of roughly $20 \mathrm{~mm}$. The remaining expandable space is filled with polyethylene. By doing this, the impact of a stepped down BSTS on the particle physics of the experiment will be shown. Table 4.7 show the results in terms of particle exiting the various location of the MBS.

Table 4.7: Study 4 Results

\begin{tabular}{cccc}
\hline \hline SS Radius(mm) & $\#$ n Exit Sides & \# g (100kev+) Exit Front & \# e Exit Front \\
\hline 429 & 554 & 2732 & 3246 \\
450 & 597 & 2415 & 3234 \\
470 & 599 & 2271 & 3257 \\
490 & 671 & 2183 & 3257 \\
510 & 747 & 2174 & 3255 \\
\hline
\end{tabular}

Stainless Steel (SS), Neutrons (n), Gammas (g), Electrons (e)

Likewise, the percent change with reference to the baseline geometry is displayed in Table 4.8. There is a drastic decrease in neutrons exiting the sides of the MBS, 
which ranges from $45.0-59.2 \%$. There is a decrease in gammas (100kev+) exiting the front of the MBS as the front radius of the BSTS is increased. Although both the gammas and neutron rates decrease, the electron rates exiting the front of the MBS increase minimally.

Table 4.8: Study 4 Percentage Change

\begin{tabular}{cccc}
\hline \hline SS Radius $(\mathrm{mm})$ & \% Change $\mathrm{n}$ & \% Change $\mathrm{g}(100 \mathrm{kev}+)$ & \% Change e \\
\hline 429 & $-59.2 \%$ & $-4.1 \%$ & $+2.9 \%$ \\
450 & $-56.0 \%$ & $-15.2 \%$ & $+2.5 \%$ \\
470 & $-55.9 \%$ & $-20.3 \%$ & $+3.2 \%$ \\
490 & $-50.6 \%$ & $-23.4 \%$ & $+3.2 \%$ \\
510 & $-45.0 \%$ & $-23.7 \%$ & $+3.2 \%$ \\
\hline \multicolumn{4}{l}{ Stainless Steel (SS), Neutrons (n), Gammas (g), Electrons (e) }
\end{tabular}

Study 5 mimics study 4 in that the back end radius of the BSTS is kept constant and the front end radius is incremented. The difference between the two is that the back end radius is now kept constant at $450 \mathrm{~mm}$ instead of $429 \mathrm{~mm}$. Likewise, the front end radius is incremented in roughly $20 \mathrm{~mm}$ increments from $429 \mathrm{~mm}$ to $510 \mathrm{~mm}$. The remaining expandable space in each of the simulations is filled with polyethylene. Table 4.9 depicts the results of Study 5 in terms of particles exiting there respective locations.

Table 4.9: Study 5 Results

\begin{tabular}{cccc}
\hline \hline SS Radius $(\mathrm{mm})$ & \# n Exit Sides & \# g (100kev+) Exit Front & \# e Exit Front \\
\hline 429 & 568 & 2750 & 3235 \\
450 & 602 & 2403 & 3233 \\
470 & 598 & 2281 & 3257 \\
490 & 682 & 2184 & 3248 \\
510 & 758 & 2173 & 3252 \\
\hline \multicolumn{2}{r}{ Stainless Steel (SS), Neutrons (n), Gammas (g), Electrons (e) }
\end{tabular}

Similarly, Table 4.10 show the percentage change referenced back to the baseline geometry simulation. The results are very similar to the study 4 result because 
all three of the targeted particles follow the same trends. The neutrons exiting the sides have the largest decreasing trend which ranges from 44.2-58.2\% decrease. Once again the gammas $(100 \mathrm{kev}+)$ exiting the front of the MBS have a decreasing trend as the radius of the stainless steel in the front is increased. Finally, the electrons exiting the front of the MBS have a slight increase.

Table 4.10: Study 5 Percentage Change

\begin{tabular}{cccc}
\hline \hline SS Radius $(\mathrm{mm})$ & \% Change $\mathrm{n}$ & \% Change g (100kev+) & \% Change e \\
\hline 429 & $-58.2 \%$ & $-3.5 \%$ & $+2.5 \%$ \\
450 & $-55.7 \%$ & $-15.7 \%$ & $+2.5 \%$ \\
470 & $-56.0 \%$ & $-19.9 \%$ & $+3.2 \%$ \\
490 & $-49.8 \%$ & $-23.3 \%$ & $+2.9 \%$ \\
510 & $-44.2 \%$ & $-23.7 \%$ & $+3.1 \%$ \\
\hline \multicolumn{2}{l}{ Stainless Steel (SS), Neutrons (n), Gammas (g), Electrons (e) }
\end{tabular}

Following the same trend as studies 4 and 5, study 6 keeps the back end radius of the BSTS constant, but this time it is set to $470 \mathrm{~mm}$. Similarly, the front end radius of the BSTS is incremented in steps of roughly $20 \mathrm{~mm}$ from $429 \mathrm{~mm}$ to $510 \mathrm{~mm}$ and the remaining expandable space is filled with polyethylene. Table 4.11 shows the results from the study.

Table 4.11: Study 6 Results

\begin{tabular}{cccc}
\hline \hline SS Radius $(\mathrm{mm})$ & $\#$ n Exit Sides & $\#$ g $($ 100kev+) Exit Front & \# e Exit Front \\
\hline 429 & 572 & 2732 & 3252 \\
450 & 618 & 2406 & 3230 \\
470 & 623 & 2277 & 3255 \\
490 & 706 & 2182 & 3248 \\
510 & 786 & 2160 & 3251 \\
\hline
\end{tabular}

Stainless Steel (SS), Neutrons (n), Gammas (g), Electrons (e)

From Tables 4.11 and 4.12 it can be seen that study 6 essentially reproduces the results that were seen in both study 4 and study 5 . The highest decrease was seen in neutrons exiting the sides of the MBS, while there is a significant decrease 
in gammas (100kev+) exiting the front of the MBS. There is minimal change in the electrons exiting the front of the MBS.

These three studies showed that eliminating the lead component in the MBS would be beneficial to the experiment. The results show that better absorption results can be found by using only stainless steel and polyethylene. The only particle absorption that did not improve was electrons exiting the front of the MBS, but because that was such a minimal increase most of it can be attributed to error within the simulations. Eliminating the lead component would also help reduce the overall weight of the MBS. Therefore, there are support structure and particle physics benefits from removing this component.

\begin{tabular}{cccc}
\multicolumn{4}{c}{ Table 4.12: Study 6 Percentage Change } \\
\hline \hline SS Radius(mm) & \% Change n & \% Change g (100kev+) & \% Change e \\
\hline 429 & $-57.9 \%$ & $-4.1 \%$ & $+3.1 \%$ \\
450 & $-54.5 \%$ & $-15.5 \%$ & $+2.4 \%$ \\
470 & $-54.1 \%$ & $-20.0 \%$ & $+3.2 \%$ \\
490 & $-48.0 \%$ & $-23.4 \%$ & $+2.9 \%$ \\
510 & $-42.1 \%$ & $-24.2 \%$ & $+3.0 \%$ \\
\hline \multicolumn{4}{r}{ Stainless Steel (SS), Neutrons (n), Gammas (g), Electrons (e) }
\end{tabular}

Figure 4.2 shows how the back end of the MBS was changed throughout these three studies. In all the studies the red and grey represent polyethylene and stainless steel, respectively. Likewise, the front end stainless steel is incremented for all the studies, although it is shown as a constant in the figure. The back end for study four is in yellow which represents the stainless steel. The rest of the back end for this study should be filled with polyethylene (red). Study five's back end stainless steel would be comprised of both the yellow and blue pieces, while the rest of the available space radially would be filled with polyethylene. Finally, the back end stainless steel for study six would include the yellow, blue, and green pieces in the 
figure with the remaining space being filled with polyethylene, like shown in Figure 4.2 .

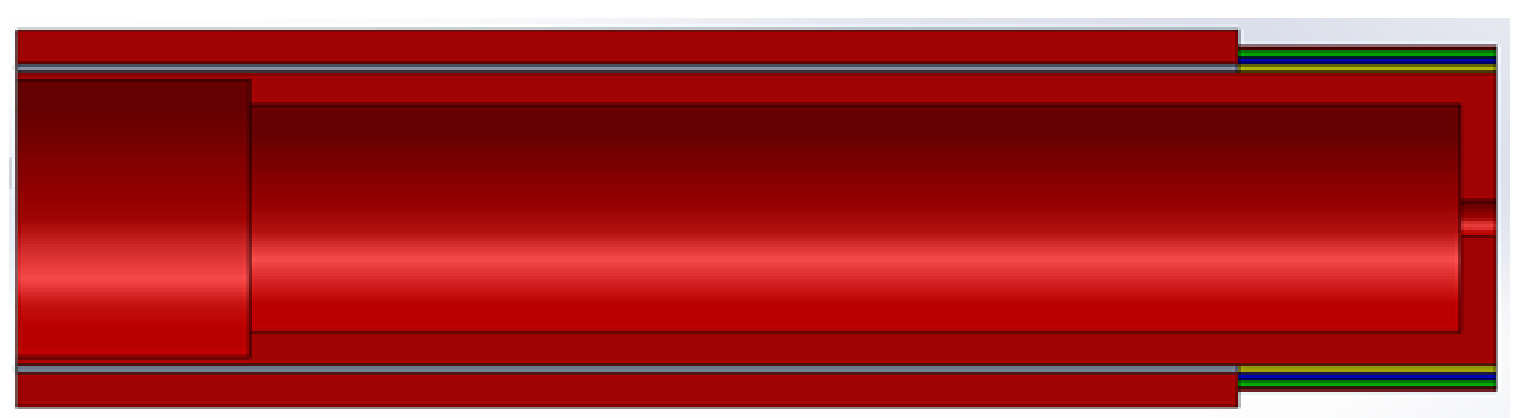

Figure 4.2: Identifying the Iterations of the MBS

\subsection{Additional Studies and Conclusions}

Upon the results of the first six studies on the MBS geometry, several additional studies were run by Dr. David Hedin, a professor in the Department of Physics at Northern Illinois University. These studies explored new geometries of the MBS using 316L stainless steel and high density polyethylene exclusively. This is due to the fact that the previous studies showed that removing the lead from the MBS provides to be beneficial both structurally and from an absorption standpoint.

One of the first proposed designs run was a layered 316L stainless steel and polyethylene MBS, shown in Figure 4.3. This approach showed much improvement in terms of particle absorption but proved to be extremely heavy with a weight reaching nearly $13,000 \mathrm{~kg}$. Another downfall of using a layered approach is the complication of assembly and maintaining the tolerance standpoint. In the figure, red denotes the polyethylene and grey denotes the steel. Just from visual inspection, it can be seen that this would be extremely difficult to manufacture and assemble. 
Each of these pieces would come with a defined tolerance, which would lead to a stacking of tolerances that would add up to a significant amount. Likewise, assembly would be arduous considering the 3 layers of steel and 3 layers of polyethylene. In the end, this configuration was deemed to be a very unlikely candidate for a new MBS.

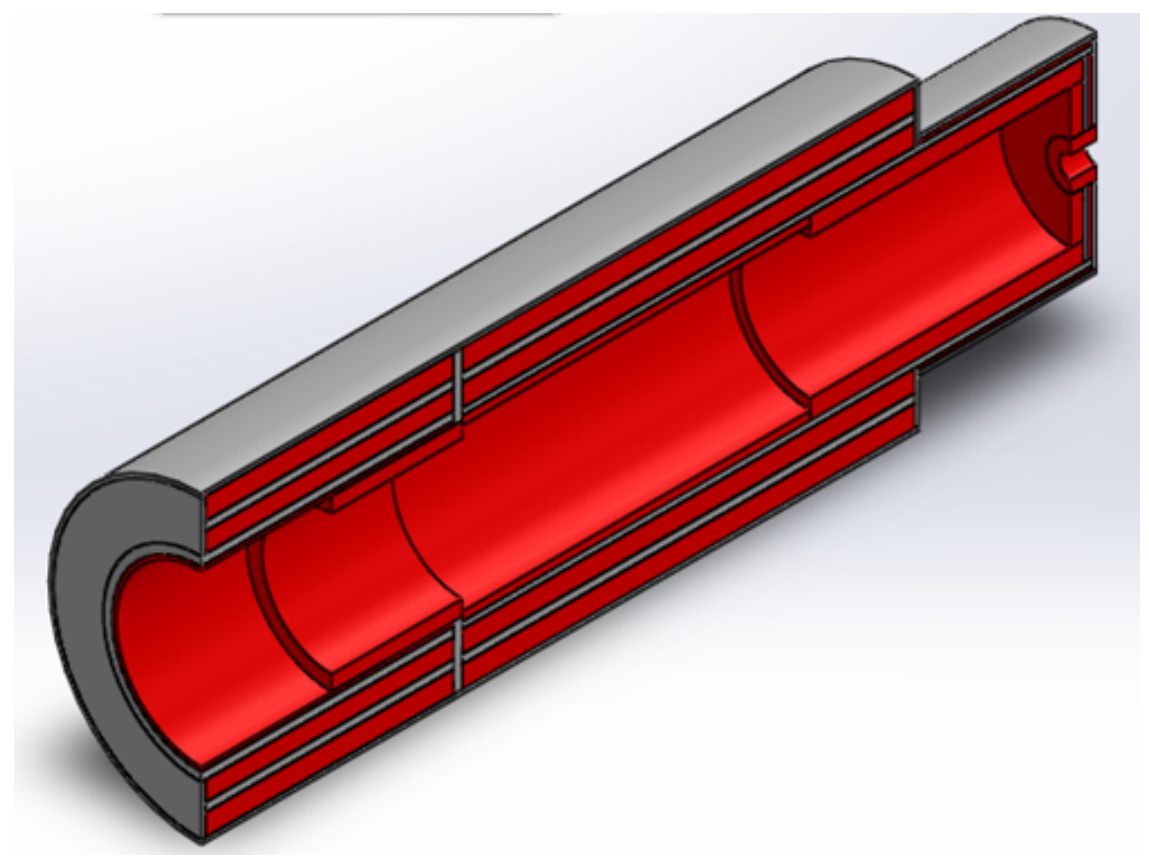

Figure 4.3: Layered 316L Stainless Steel and Polyethylene MBS

After realizing how much mass is added to the MBS by exploring a layered approach, the next step was to try to find a configuration that would satisfy one of the three mass constraints in Section 2.3. A configuration was found that included the same inner polyethylene components as the baseline geometry, but added a large steel ring at the front of the MBS. Also, there was another steel ring in the front half of the MBS that extended to on outer diameter of $1400 \mathrm{~mm}$ and a thickness of $92 \mathrm{~mm}$. There are also two polyethylene rings that surround the main steel tube. Figure 4.4 shows the potential configuration. 


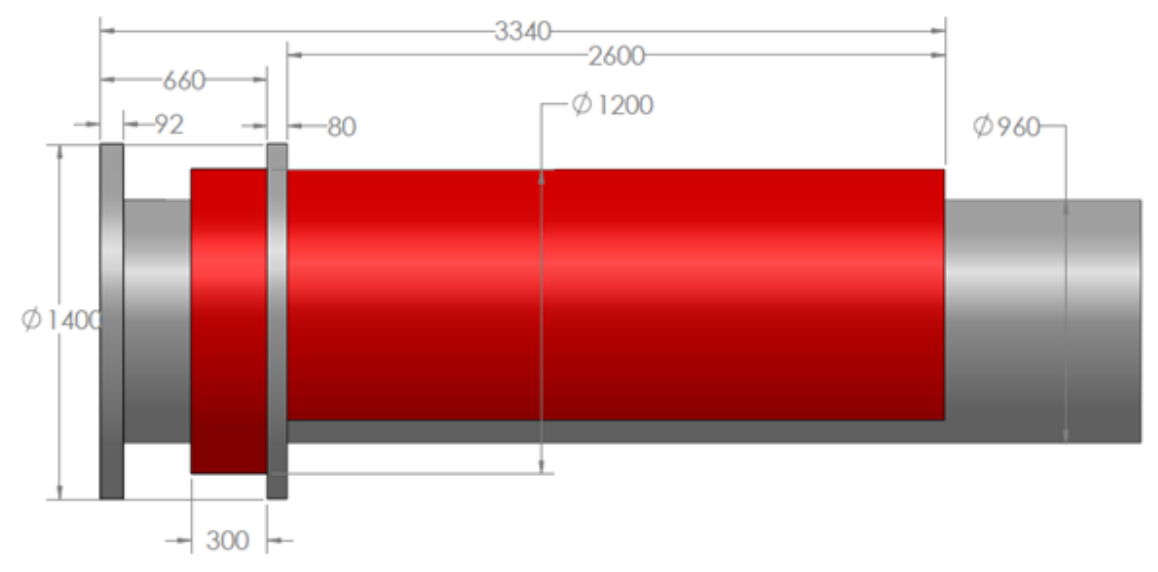

Figure 4.4: MBS Satisfying Constraints

In order to meet the mass constraints several measures had to be taken. First, the large steel rings are a necessity because those rings move the center of mass forward. Likewise, it can be seen that the polyethylene on the outside is at a smaller diameter than the steel. This was done in order to lower the mass. Finally, the polyethylene at the back end was modified in order to lower the mass and move the center of mass forward. To further reduce mass, the bottom $45^{\circ}$ of the polyethylene was eliminated. Even with all of these modifications, the mass and center of mass constraints were barely met. The mass of this configuration is $4447.68 \mathrm{~kg}$ and the center of mass is $2501.34 \mathrm{~mm}$ from the back of the MBS. Therefore, this cleared the mass constraint by $32.32 \mathrm{~kg}$ and cleared the center of mass constraint by $1.34 \mathrm{~mm}$. In the end, this configuration is very impracticable due to the limited space in the front end for support.

Finally, a configuration was settled on, and is made completely out of $316 \mathrm{~L}$ stainless steel and high density polyethylene. This configuration does not meet the initial mass or center of mass constraints but upon discussion with Fermilab engineers and physicists this configuration was deemed acceptable. Figure 4.5 shows a section view the configuration that was settled on. This configuration consists of 
a constant stainless steel tube down the entire length of the MBS, two support rings that improve the stiffness of the MBS, fully expanded polyethylene on the outside of the MBS, and internal polyethylene pieces that prevent back scatter.

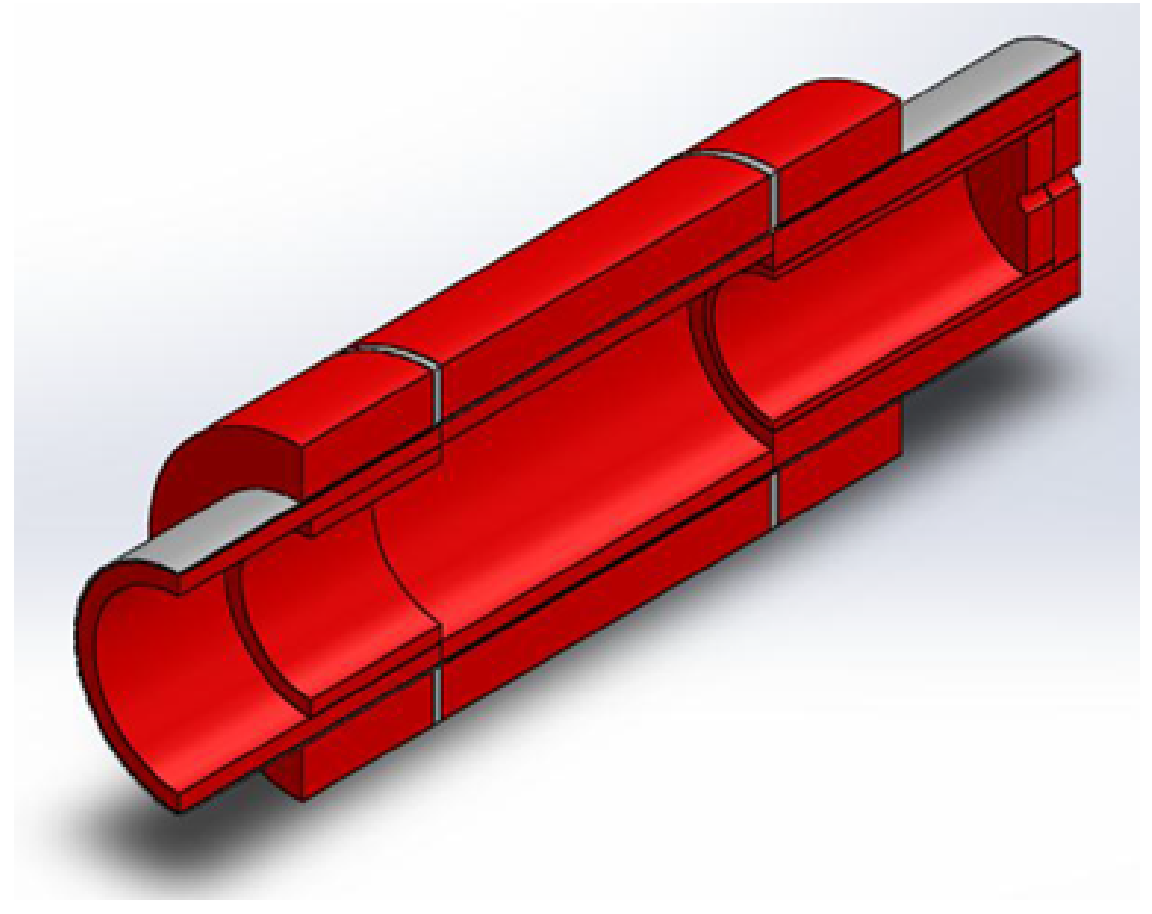

Figure 4.5: Final MBS

The mass of this configuration is approximately $4900 \mathrm{~kg}$ with a center of mass around $2040 \mathrm{~mm}$ from the back of the MBS. Since the mass constraints are acceptable, this configuration was evaluated through G4Beamline. The particle physics results of this configuration are shown in Table 4.13.

\begin{tabular}{|c|c|c|c|}
\hline & Neutrons & Gammas (100kev+) & Electrons \\
\hline \# of Particles & 296 & 2276 & 2782 \\
\hline \% Change & $-78.2 \%$ & $-20.1 \%$ & $-11.8 \%$ \\
\hline
\end{tabular}

This table shows both the number of each particles exiting there designated areas as well as the percent change from the baseline geometry. The neutron and 
electron results are the best seen by any of the simulations run by far, at decrease of $78.2 \%$ and $11.8 \%$ respectively, when compared to the original geometry. The gamma results are slightly less than the best results seen, and only differ by around $4 \%$.

Initially, the lead provided more space at the back end of the MBS, but after further analysis it was concluded that it added unnecessary weight and toxic material. Given the combination of stainless steel and polyethylene, several different geometries were considered throughout the studies, this geometry provided the best balance of particle absorption. All of the particle rates decrease when compared to the original geometry, and the neutron and electron rates were the best results seen by any of the geometries simulated. A final engineering drawing of the MBS is provided in Appendix A. 


\section{CHAPTER 5 ENGINEERING CONSIDERATIONS}

Taking the final geometry settled on from a particle physics standpoint, engineering need to be done to ensure that it is possible to support and assemble this configuration. First, the mass and center of mass will be looked at, which will dictate, to an extent, which one of the three support options will be chosen. Likewise, the chosen support option will push the assembly procedure because each support option has unique features, such as a one or two piece MBS.

\subsection{New MBS Measurables}

This newly settled on MBS has a mass just under $4900 \mathrm{~kg}$ and a center of mass around $2040 \mathrm{~mm}$ from the back of the MBS. Comparing these statistics back to the mass constraints defined in Section 2.3, there is not one of the options in which both the mass and center of mass are satisfied. Options one and three are both satisfied from a mass standpoint, while both the constraints in option two are not satisfied. This narrows the support option down to either option one or three. The center of mass constraint for option one is that the center of mass must be at least $3000 \mathrm{~mm}$ from the back of the MBS, while option three provides a window of 2750-2250 mm from the back of the MBS. Therefore, option three where the MBS is supported off both MBS supports and the IFB would be the leading candidate to support the MBS. 
Although, the center of mass of the new MBS is only off by about $210 \mathrm{~mm}$ it is very difficult to shift this center of mass while still keeping the mass constraint. Looking back to Section 4.5, one can see a MBS configuration that met both mass and center of mass constraints, but this was done by adding a large stainless steel ring, $1400 \mathrm{~mm}$ outer diameter and $92 \mathrm{~mm}$ thick, to the front of the MBS. Upon further discussions, the center of mass of this new MBS was deemed acceptable due to the improve physics. Likewise, future considerations will be taken in order to shift this center of mass closer to the constraint's center of mass. This can be done by removing polyethylene on the bottom of the MBS, as well as possibly thinning the back support ring and thickening the front support ring.

The new MBS also meets the expandable space constraints, as it stays within these bounds. The outer dimensions along the axis of the MBS is shown in Figure 5.1. The front of the MBS starts at an outer diameter of $960 \mathrm{~mm}$ for the first $560 \mathrm{~mm}$. Then the MBS is expanded out to the maximum outer diameter of $1400 \mathrm{~mm}$ for $2740 \mathrm{~mm}$ along the axis. Likewise, the back end of the MBS is brought back down to a diameter of $960 \mathrm{~mm}$ for the final $816 \mathrm{~mm}$.

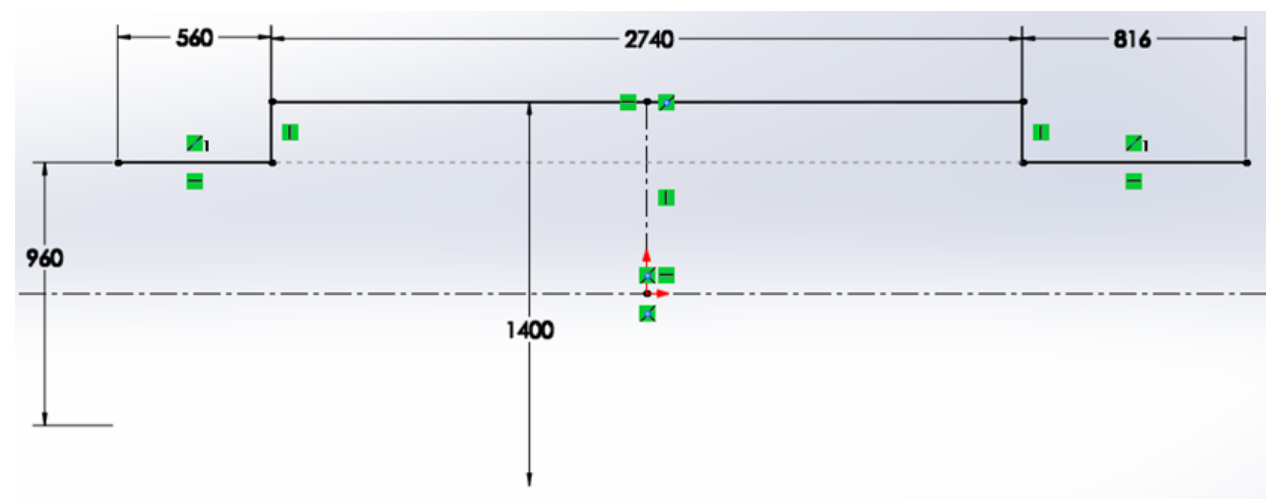

Figure 5.1: Outer Dimensions of the New MBS 


\section{$5.2 \quad$ Support Structure}

Since, the third option of supporting the MBS was chosen, the MBS will have front MBS supports and back IFB support. The MBS will be supported by two pins on the IFB. One of the pins will be fixed, while the other will be free to rotate about the X-axis. The front end support will utilize a gimballed support that will allow rotation about the Y-axis. Figure 5.2 shows the stainless steel tube with pins attached at the back end. The front support will most likely have to include a thrust bearing, in which a pin attached to the MBS will sit within the thrust bearing. The thrust bearing should not be lubricated by liquid because it is possible for the liquid to evaporate in the high vacuum environment of the experiment.

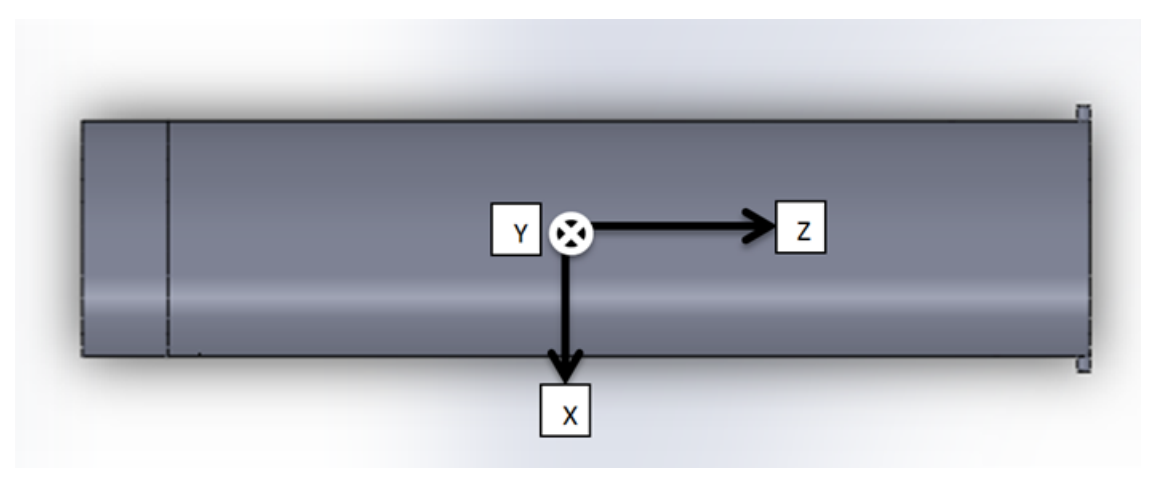

Figure 5.2: MBS with IFB Support Pins

Keeping this support setup in mind, stress analysis was performed on the IFB pins by a Fermilab engineer to ensure that these pins can withstand the forces that will be put on them by the MBS. This analysis was performed under the boundary conditions of the final MBS configuration and support structure. Figure 5.3 shows the stress results from this analysis.

This figure shows that the stress on the pins is acceptable because the maximum point of stress is just over $28.5 \mathrm{MPa}$ [14]. Coupling that information with the material 


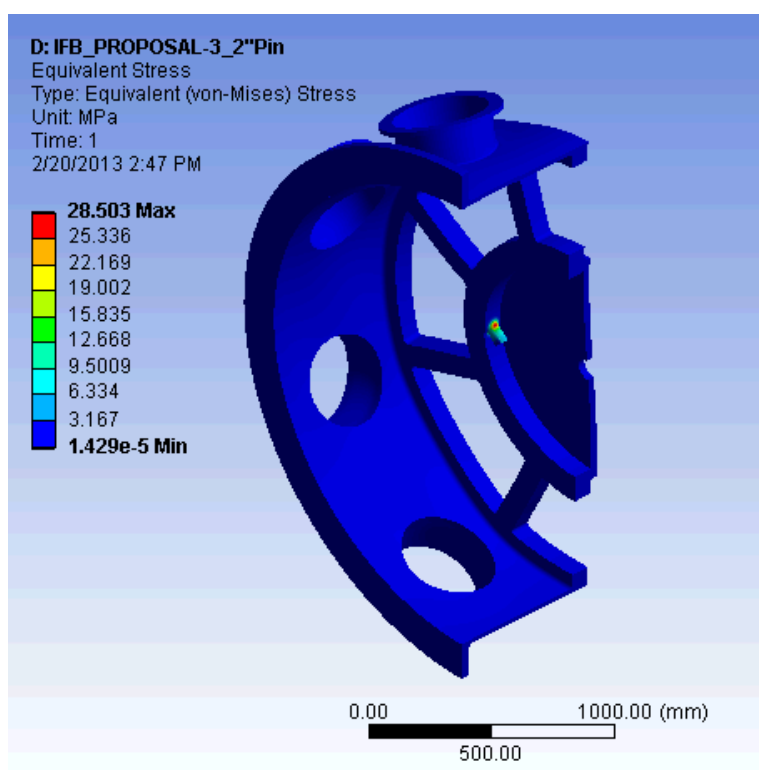

Figure 5.3: Stress Analysis on IFB Support Pins [14]

properties of 316L stainless steel, this leads to the conclusion that the factor of safety is greater than 8 for these pins which is well over the desired factor of safety of 2.5 .

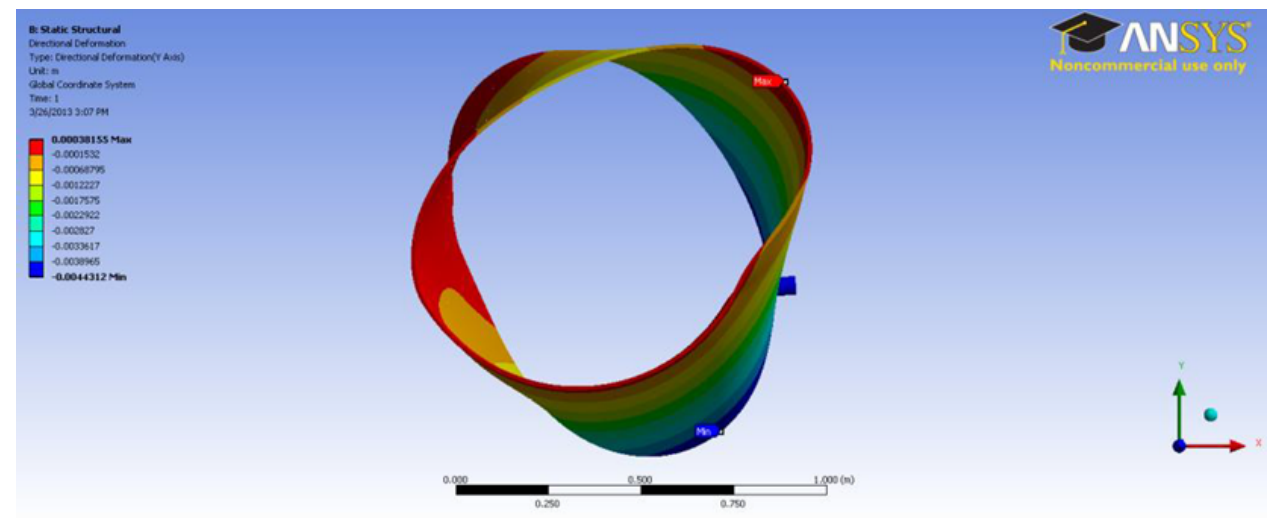

Figure 5.4: Deflection Analysis on Main Stainless Steel Tube

Likewise, deflection analysis was run on the main stainless steel MBS tube under the MBS weight and support conditions stated previously. Initially, the analysis utilized only the main MBS tube as its model. The results from this analysis can be seen in Figure 5.4. 
This analysis shows that the tube deflects about $4 \mathrm{~mm}$ in total deflection. This maximum deflection is the red areas in the figure. The deflection is quite significant especially considering that alignment is crucial for this experiment. Another iteration of this was run with a $1.5 \mathrm{~mm}$ stainless steel tube instead of the $1 \mathrm{~mm}$ stainless steel tube, and this yielded a deflection of about $1.75 \mathrm{~mm}$. Improvements can be seen in the next simulation that was run where the geometry was changed and the stiffener rings were added to the geometry. The main stainless steel tube was set at $1 \mathrm{~mm}$ for this analysis. Figure 5.5 shows this deflection analysis.

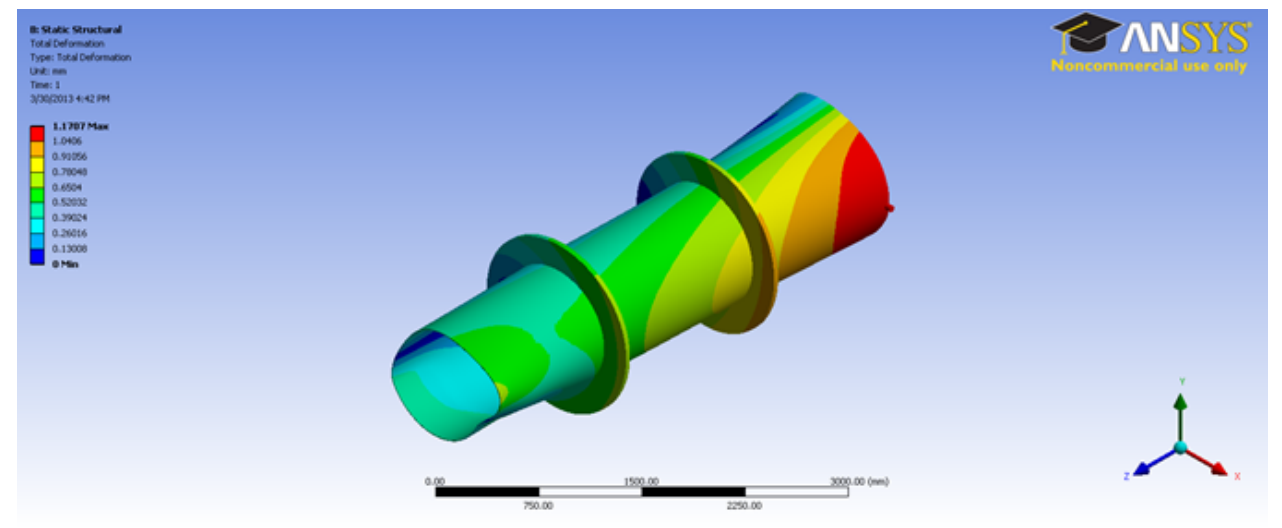

Figure 5.5: Deflection Analysis with Stiffener Rings

A $1.2 \mathrm{~mm}$ total deflection can be seen with the stiffener rings included, which are a part of the MBS final geometry. These rings extend out to an outer diameter of $1400 \mathrm{~mm}$ and are $40 \mathrm{~mm}$ thick. The $1.2 \mathrm{~mm}$ deflection is the red areas in the figure. For all of these simulations, the polyethylene was used as a force on the MBS tube. While the polyethylene will create a force on the MBS tube, it will also add to the stiffness of the structure which would lower the total deflection of the MBS. Therefore, determining the assembly procedure for the MBS is crucial. 


\subsection{Possible Assembly Procedures}

One of the main questions for the assembly procedure is whether or not it will be assembled at Fermilab or not, and if it is assembled at Fermilab whether or not there is room to assemble it vertically. Assembling vertically could prevent the MBS from binding because the MBS would not deflect radially in this state. Where as if it were assembled in a horizontal state each piece placed in the main stainless steel tube would cause some deflection. Each subsequent addition would incur more deflecting making the fitting of multiple polyethylene pieces very challenging. If this deflection interfered with the tolerance of the piece being assembled it could cause binding.

First, assuming that the MBS will be assembled vertically the internal polyethylene pieces could be individually inserted into the bore of the main stainless steel tube. On the outside, one of the stiffener rings would need to be welded or secured to the stainless steel tube and then the polyethylene could be assembled to the outside using the stiffener ring as a gage as to how far the polyethylene should slide down the tube. Further analysis should be done to determine how the stainless steel tube would deform due to the localized heat of the welding process if that route is chosen. This will also narrow down whether or not the inner polyethylene should be inserted before or after the outer pieces are assembled. After being assembled the MBS could be placed on the support structure via crane or forktruck depending on crane coverage or space for a forktruck.

If the latter were chosen, in which the MBS has to be assembled horizontally, the welding deformation still needs to be determined. An assembly deflection process should be defined so that it can be determined if the MBS will bind during assembly. 
Once defined, the inner polyethylene pieces can be safely placed into the bore of the main stainless steel tube, as well as the outer polyethylene and stainless steel pieces.

The new MBS is a viable option because the mass and center of mass constraints can be satisfied, and there is room to remove some mass if it were really needed. These characteristics provide manageable deflection for alignment and assembly, but should be looked into further for future considerations. Likewise, the assembly process should be similar to the one of the original MBS, as there are pieces that need to be inserted into the bore of the stainless steel tube and pieces that need to be inserted on the outside of the MBS. One of the new pieces to the assembly procedure is how the stiffener rings will be attached to the stainless steel tube, or if they will simply sit in place. All in all, this configuration makes improvements from a particle physics standpoint and can be supported safely. 


\section{CHAPTER 6 CONCLUSIONS}

\subsection{An Improved MBS}

Taking in all the particle physics and structural constraints, it can be concluded that there are better configurations of the MBS that can be safely supported and improve particle absorption. The results of the final MBS geometry show that the absorption of neutrons, electrons, and gammas(100kev+) are improved by large margins compared to the baseline geometry. Another major conclusion of this study is that the lead should be removed from the MBS geometry. The first three G4Beamline studies confirm this conclusion. Removing the lead lowers the mass of the MBS allowing more space and mass to be dedicated to adding stainless steel and high density polyethylene. Also, the MBS should be supported off of the IFB in the back and on MBS supports in the front. This support configuration will enable the MBS to rotate throughout the experiment.

The new MBS made exclusively of 316L stainless steel and high density polyethylene dwarfs the original MBS's particle physics results. Improvements were made in each of the three categories that were looked at in G4Beamline. Neutrons exiting the sides of the MBS reduce by $78.2 \%$, while electrons exiting the front of the MBS decrease by $11.8 \%$ when compared to the baseline geometry. Both of these categories are the best results seen by any of the results of studies 1-6. Likewise there is a $20.1 \%$ decrease in gammas $(100 \mathrm{keV}+)$ exiting the front of the MBS. Although 
this is not the best result seen when compared to results from studies 1-6, it is only off by approximately $4 \%$ from the best result.

From a structural standpoint, the MBS works with manageable deflection, about $1.2 \mathrm{~mm}$ total deflection. This was simulated using the imposed loads of the polyethylene pieces on the main stainless steel tube with stiffener plates included. If needed, this stiffness could be lowered further by thickening the stainless steel tube, which was shown to deflect about $1.75 \mathrm{~mm}$ without stiffener rings. When adding the polyethylene, stiffness will also be added to the MBS and it should deflect even less. This process could be looked into further as the experiment's timeline nears construction.

\subsection{Future Work}

Although several improvements have been made to the MBS from this study, there is still work that can be done to improve on the support structure and assembly procedure. For starters, improvements can be made on the deflection analysis done to the MBS. The deflection analysis shown earlier showed just the MBS stainless steel tube under the forces imposed by the polyethylene pieces. Although this is suitable, it could be improved by using an assembled MBS under inertial loading. This simulation could be arduous because of the fact that one would have to include contacts between different pieces, and in the end might prove to be less accurate and very time consuming. Another addition to the simulation would be to impose the forces that the MBS would see by the cabling, as this was left out of the previous simulations due to these weights not yet fully defined by Fermilab. 
Outside of improving the deflection analysis, the assembly procedure should be looked into. Now that the final geometry is fully defined, the number of polyethylene pieces still need to be looked into. Also, how these polyethylene pieces are linked together should be explored. Once these are defined, the assembly procedure should be worked on to derive a step-by-step procedure of how the MBS will be put together. Also, a gimballed support structure is currently being worked on in order to support the front end of the MBS. The support structure will be composed of stainless steel I-beams and plates. It will sit on four bearing blocks, sized properly, ensuring that the bearing blocks can withstand the front end weight. Finally, a thrust bearing is being defined that will be a part of the support structure allowing the front of the MBS to rotate throughout the length of the experiment. Although there is still much work to be done, it is anticipated that this can be accomplished by the CD2 timeline. There are still many open questions the the major hurdles regarding material and sizing are now addressed as a result of this research. 


\section{REFERENCES}

[1] Fermi National Accelerator Laboratory. Mu2e Conceptual Design Report. March 2012. Fermilab-TM-2545.

[2] Particle Physics. Particle Physics: Muons. Alternative Particle Physics. http://www.alternativephysics.org/book/Particles.htm. 4/10/2013.

[3] Fermilab National Accelerator Laboratory. Muons, Electrons and the Laws of Physics. Mu2e for the Public. http://mu2e.fnal.gov/public/gen/index.shtml. $2 / 20 / 2013$.

[4] Fermilab National Accelerator Laboratory. 1/5/2012. Why Muons at the Intensity Frontier?. Mu2e. http://mu2e.fnal.gov/why-muons.shtml. 2/20/2013.

[5] Gross, David. The role of symmetry in fundamental physics. Proceeding of the National Academy of Sciences of the United States of America. Volume 93. Number 25. http://www.pnas.org/content/93/25/14256.full. 4/11/2013.

[6] Lamm, Michael. Mu2e Production Solenoid Requirements Document. March 6, 2012. Mu2e-docdb-945.

[7] Lamm, Michael. Mu2e Transport Solenoid Requirements Document. March 17, 2012. Mu2e-docdb-947.

[8] Lamm, Michael. Mu2e Detector Solenoid Requirements Document. April 25, 2012. Mu2e-docdb-946.

[9] Bossert, Rodger. WBS 5.8 - Muon Beam Stop. May 20, 2012. Mu2e-doc-1351. 
[10] Bossert, Rodger. WBS 5.10 - Detector Support and Installation System. May 20, 2012. Mu2e-doc-1383.

[11] Puccinelli, Federico. Detector Solenoid Rail System Analysis. November 1, 2011. Mu2e-doc-1991.

[12] Puccinelli, Federico. Muon Beam Stop Support Structure Analysis. November 1, 2011. Mu2e-doc-1992.

[13] Gallo, Giuseppe. MBS Conceptual Design. February 7, 2013. Mu2e Document 2779-v1.

[14] Gallo, Giuseppe. Muon Beam Stop Support Design. March 5, 2013. Mu2e Document 2786-v1.

[15] Robert, Tom. Muons, Inc. G4Beamline User's Guide. November 2012.

[16] Geant 4. 3/28/2013. Geant 4. http://geant4.cern.ch/. 4/15/2013.

[17] Khalatian, Vladimir. Mu2e G4Beamline Manual. January 24, 2012. Mu2e Document 1490-v7.

[18] Measday, D.F. The nuclear physics of muon capture. Physics Reports. December 2000. Mu2e Document Database. Web. 4/15/2013. 
APPENDIX A

FINAL MBS CONFIGURATION 


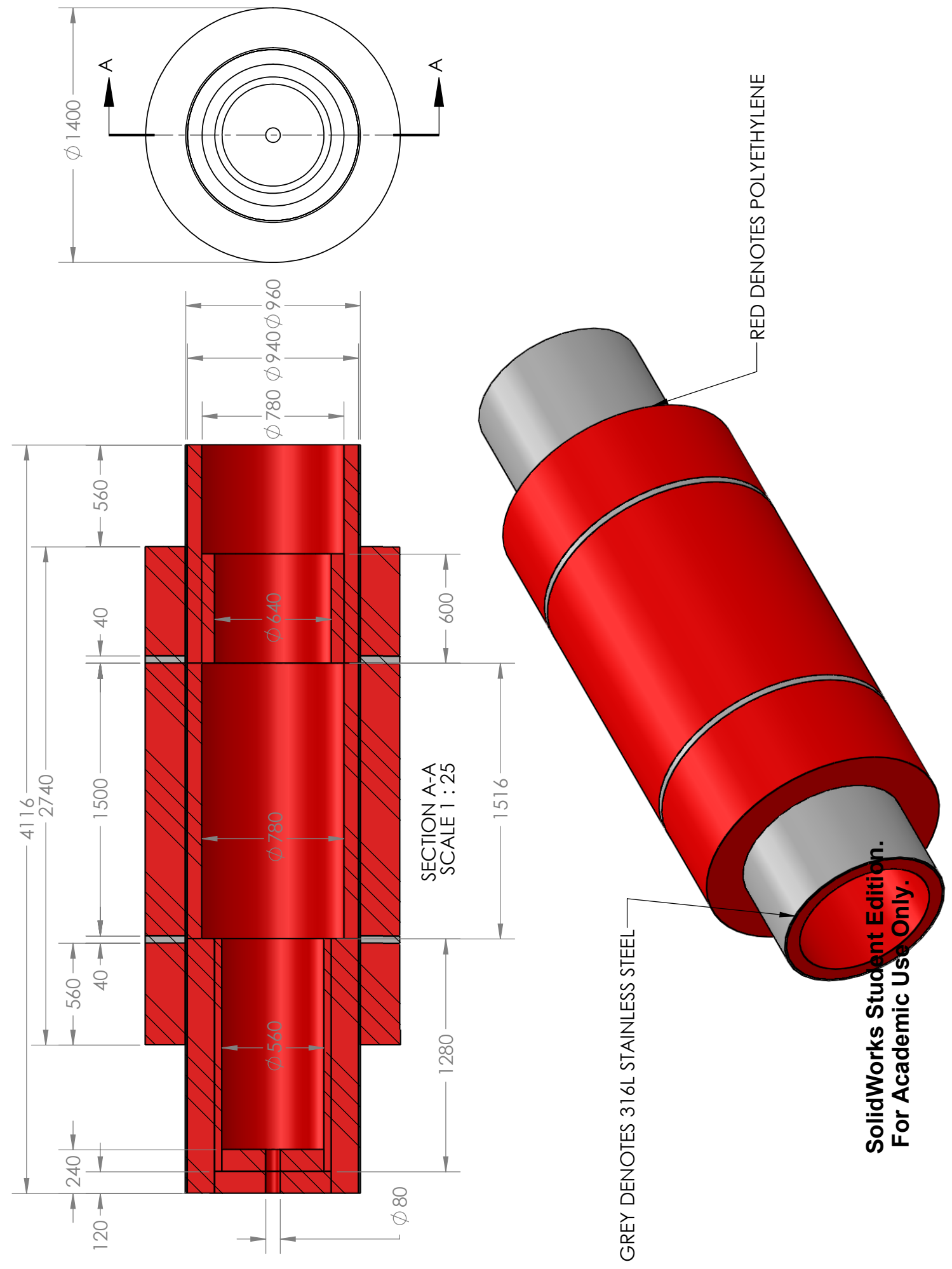


APPENDIX B

G4BEAMLINE INPUT FILE 


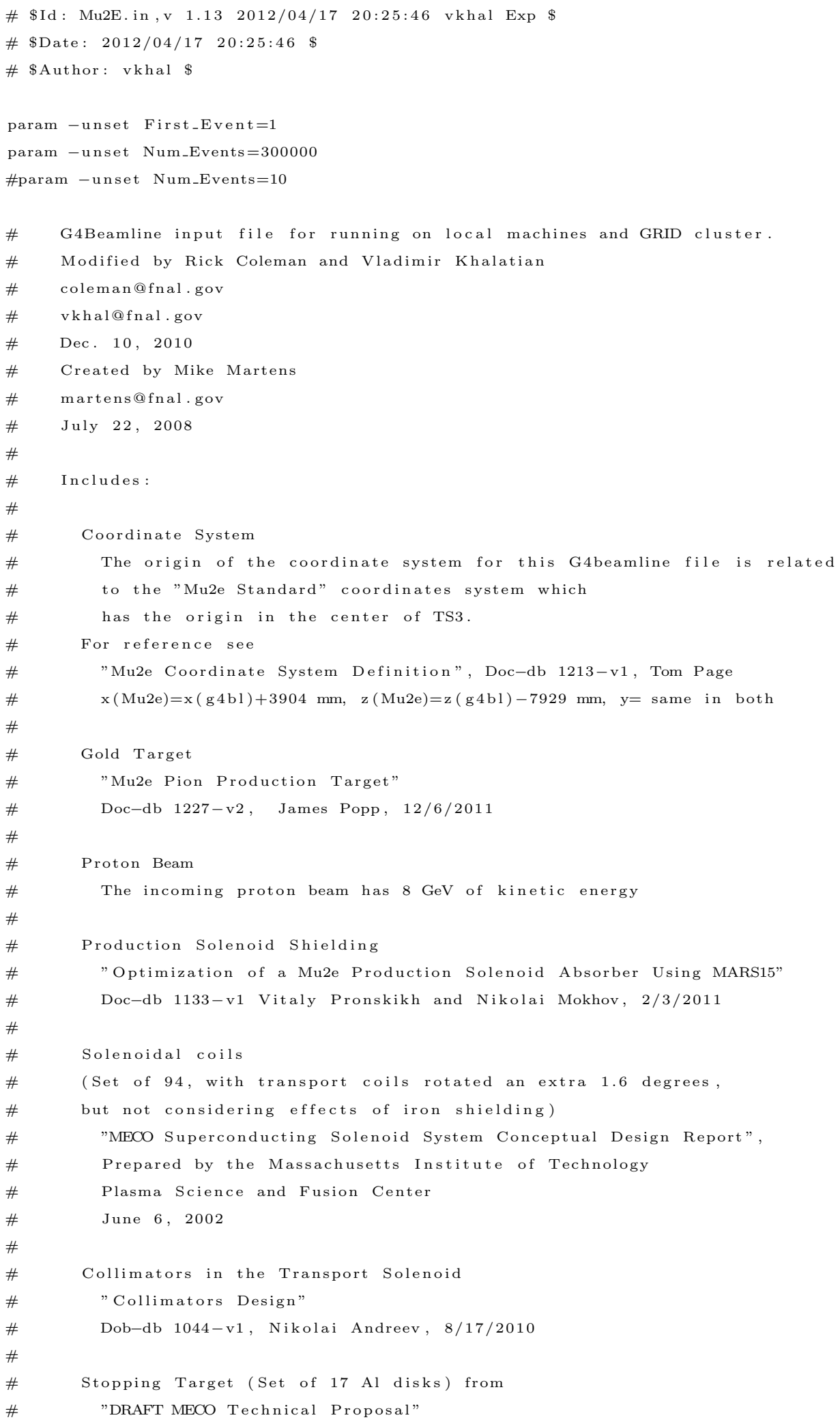




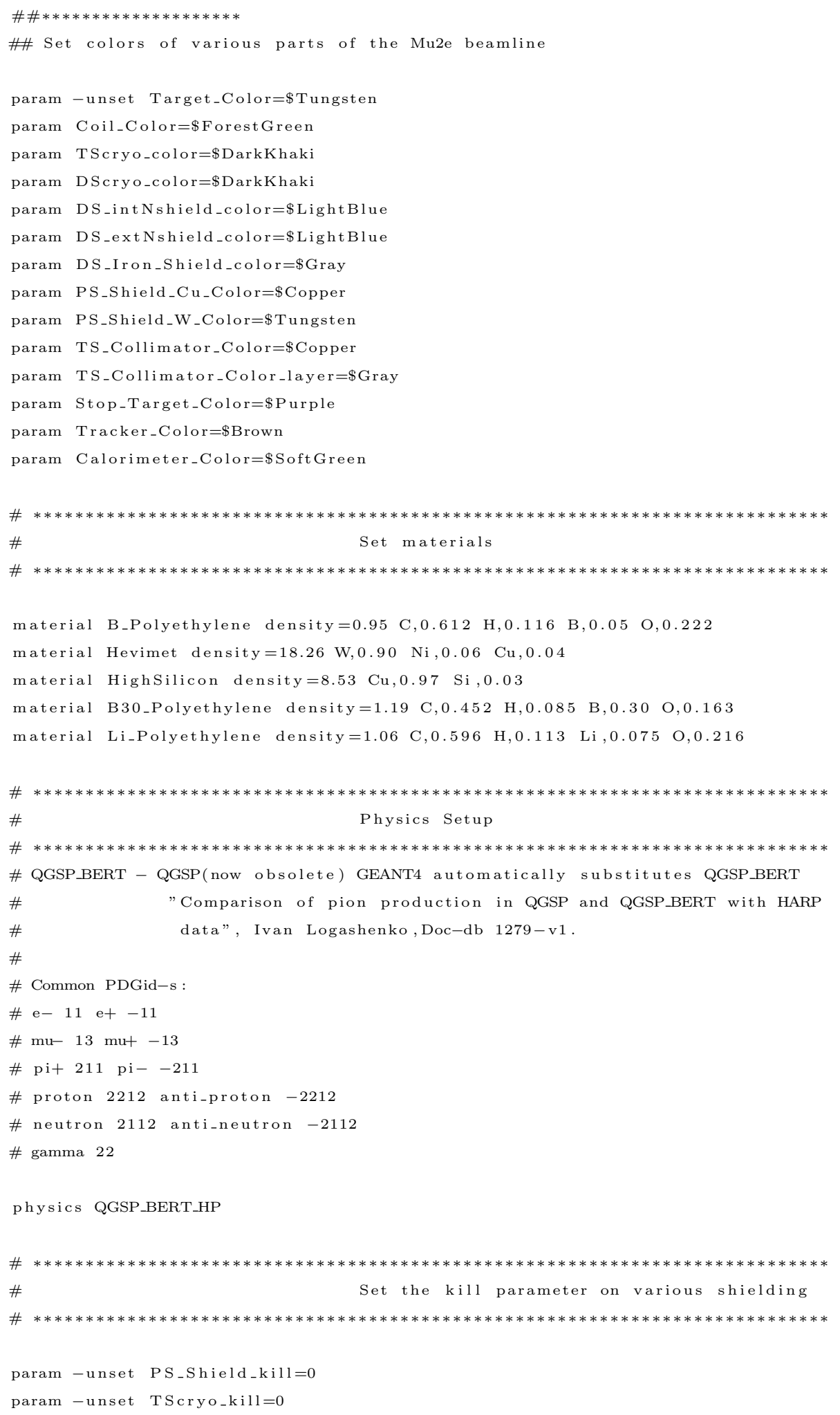




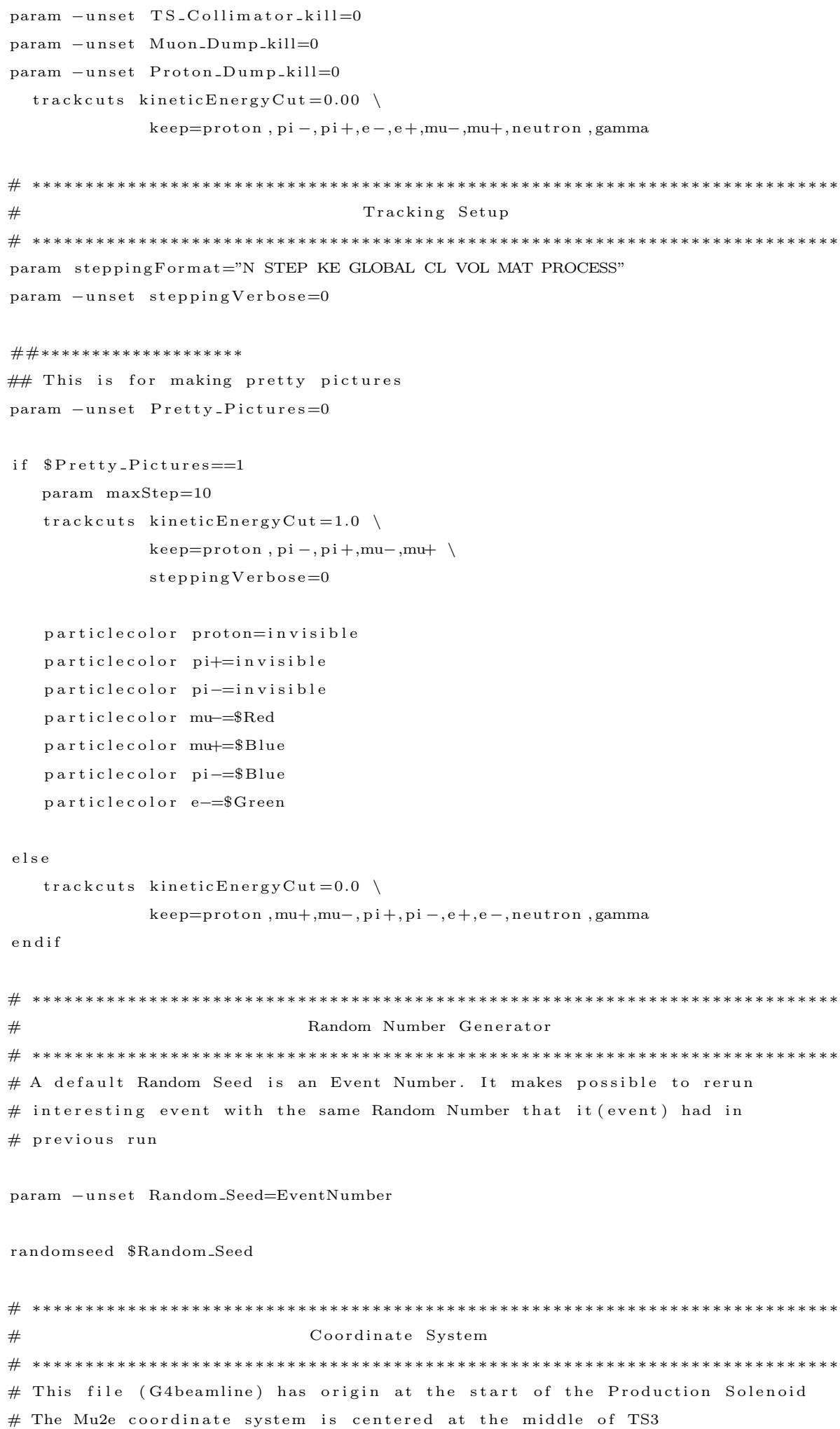




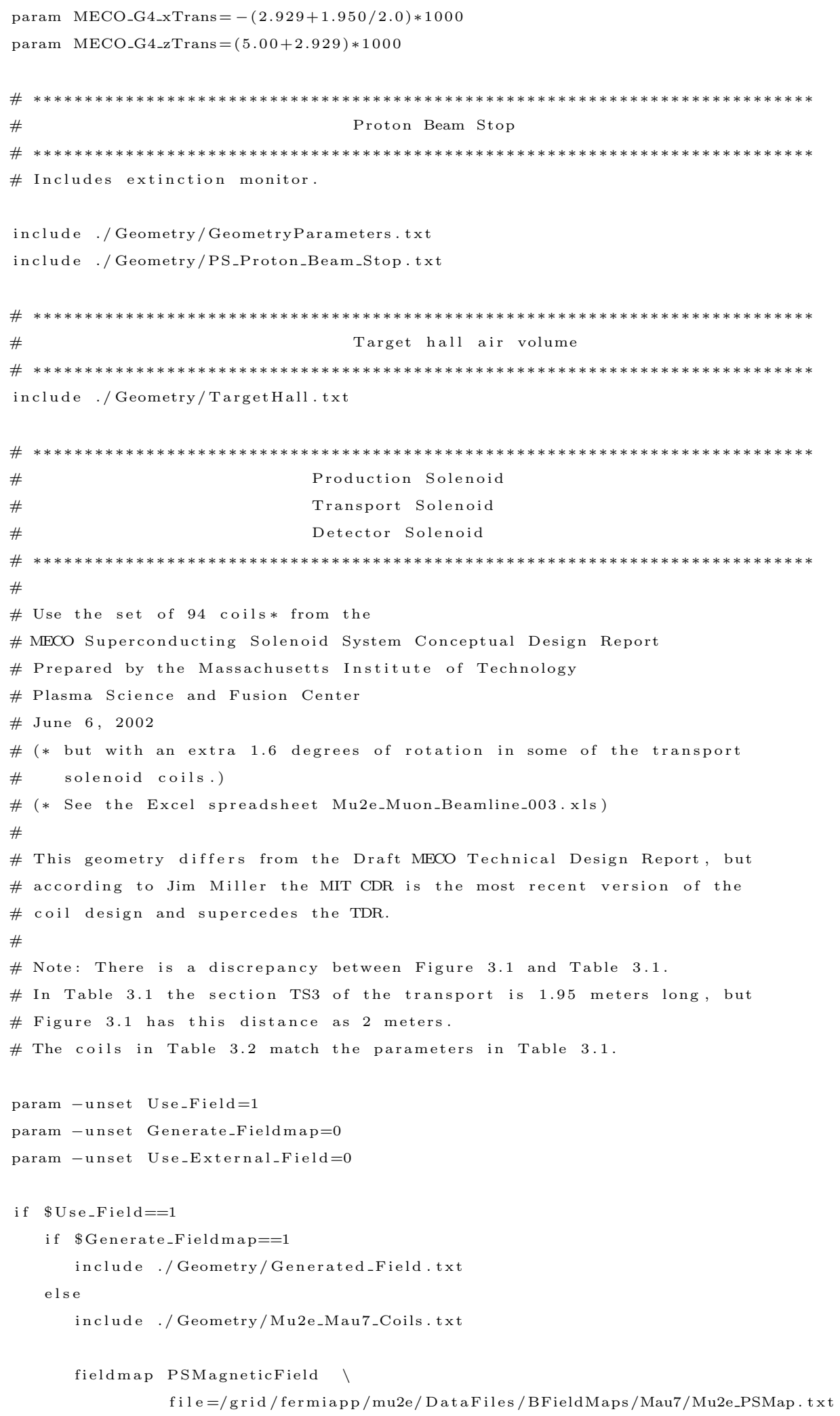




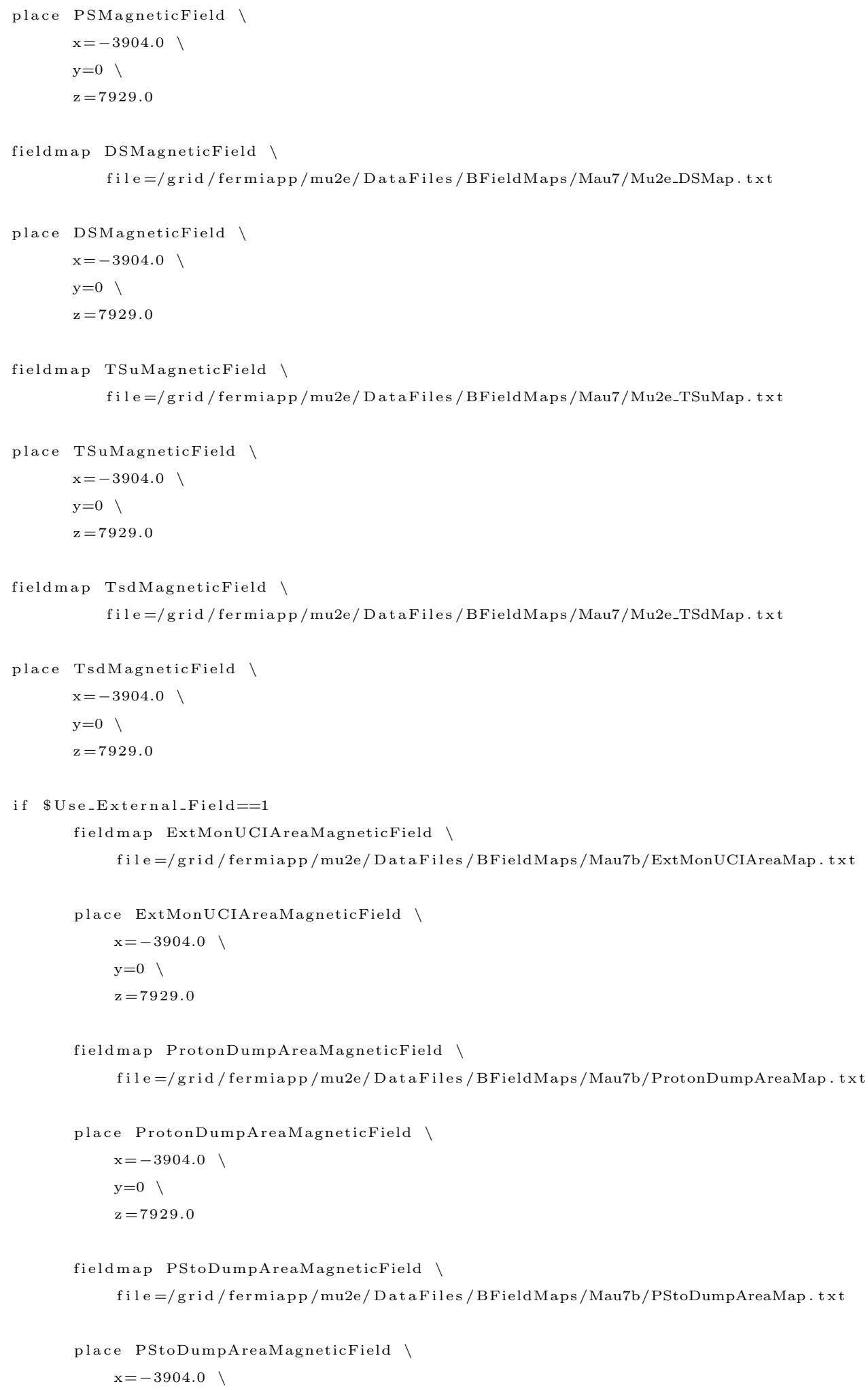




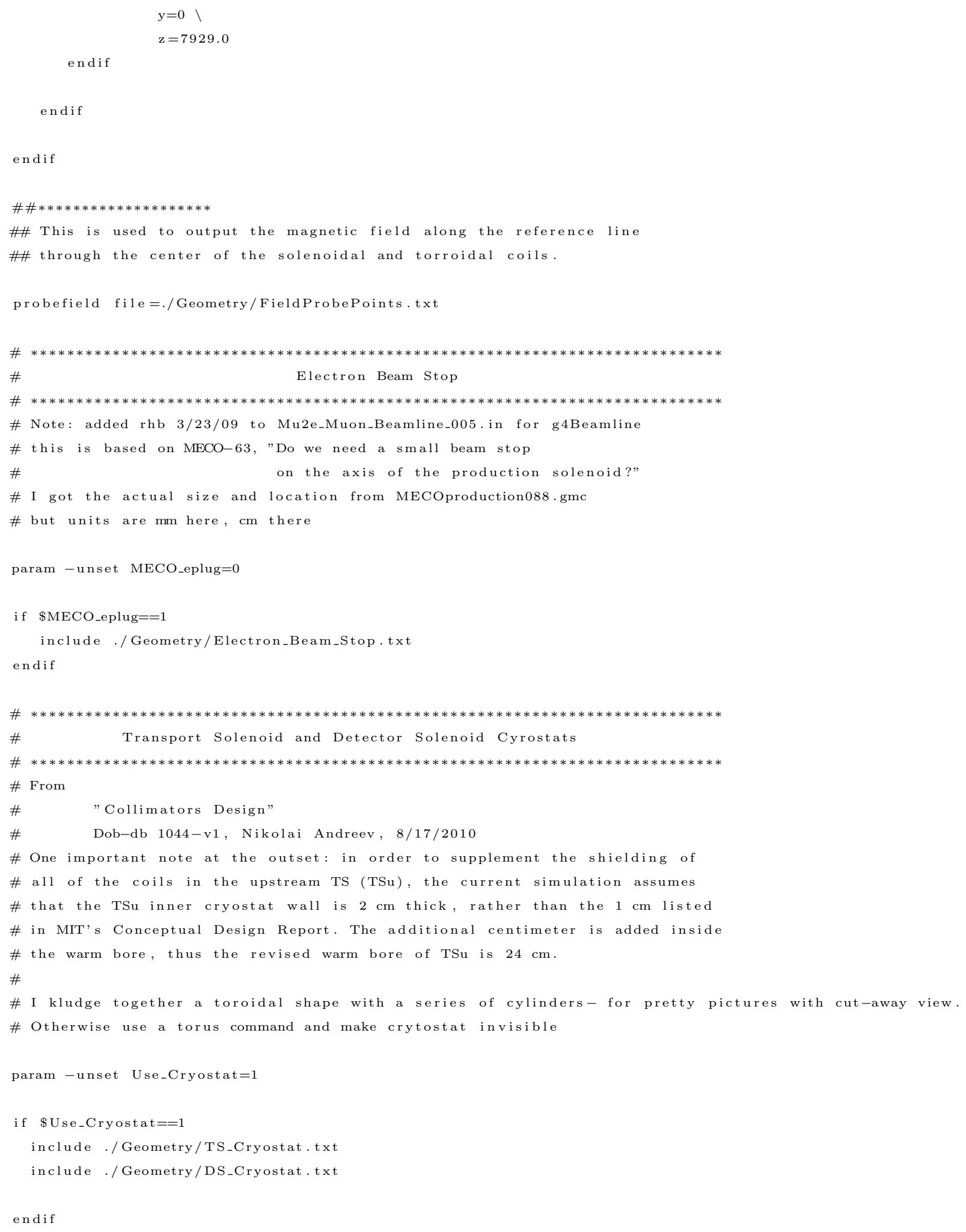




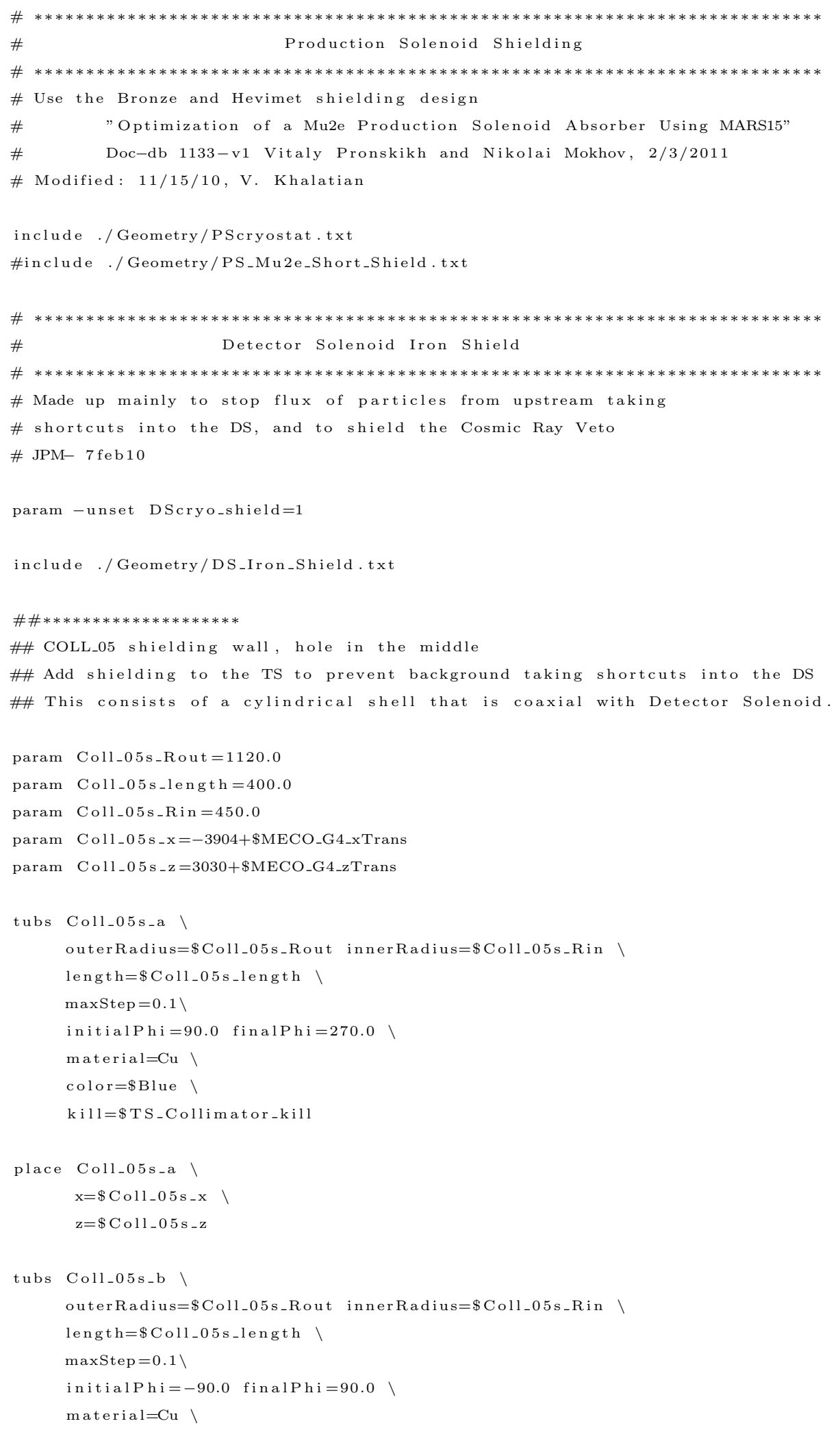




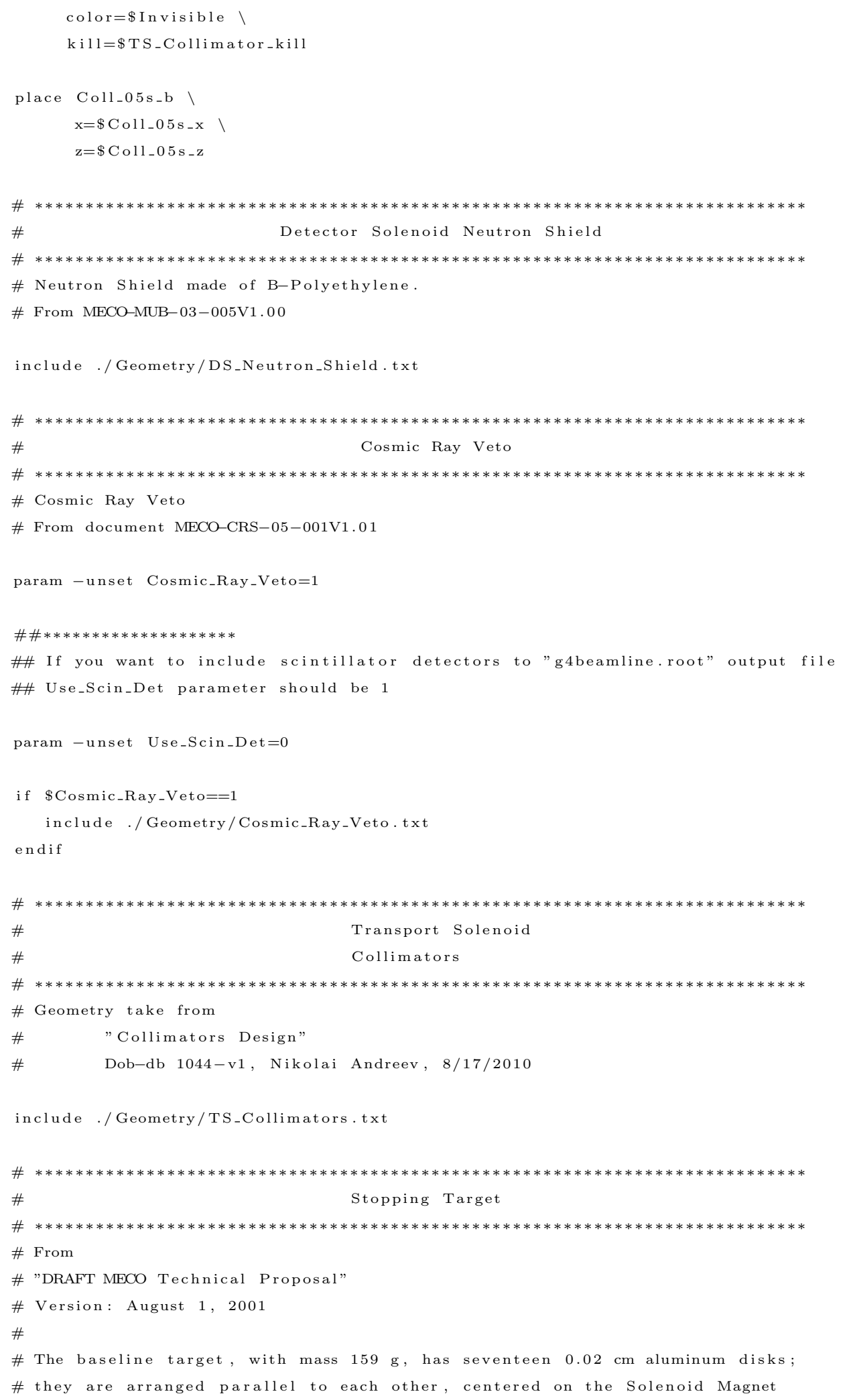




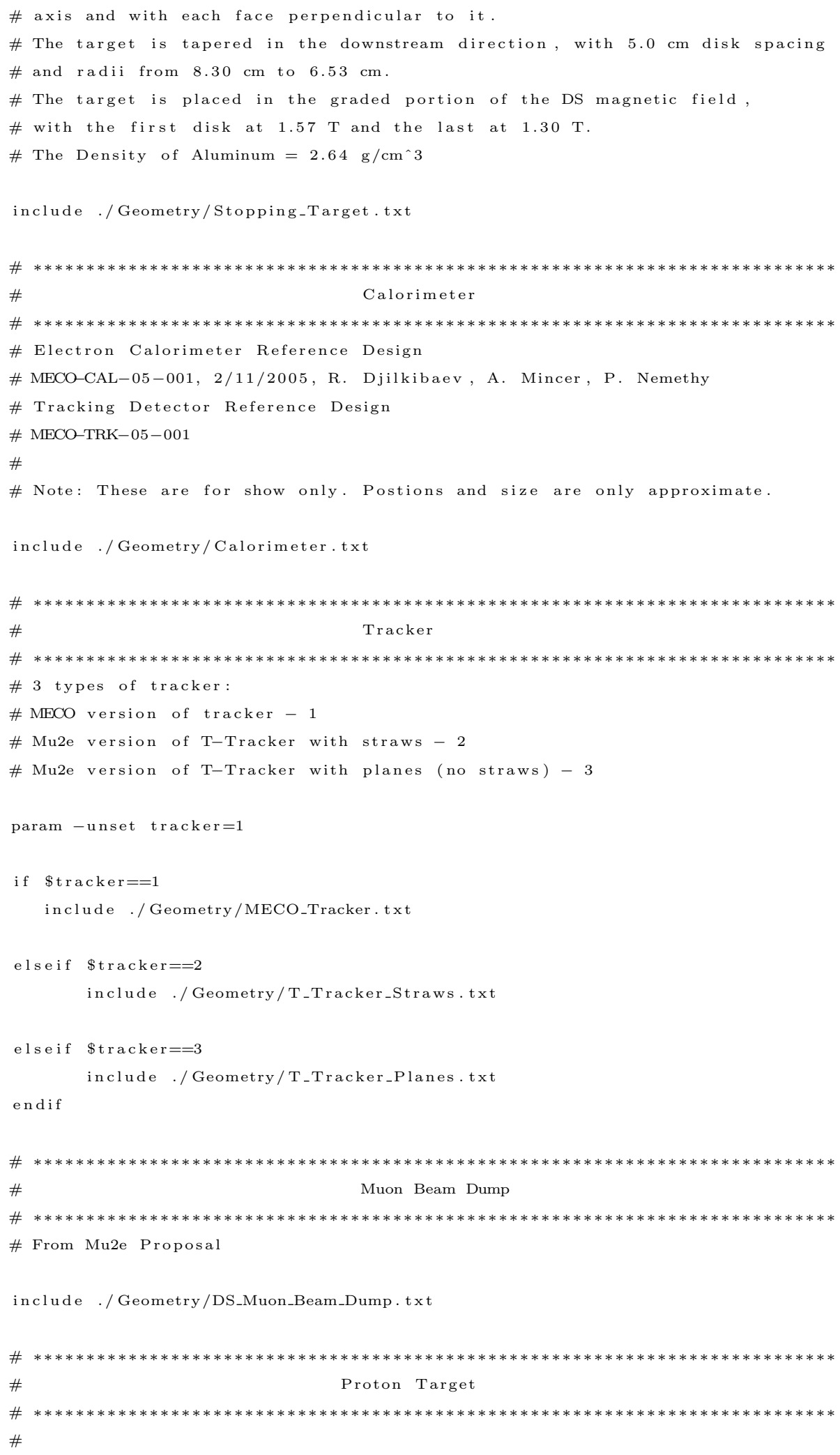




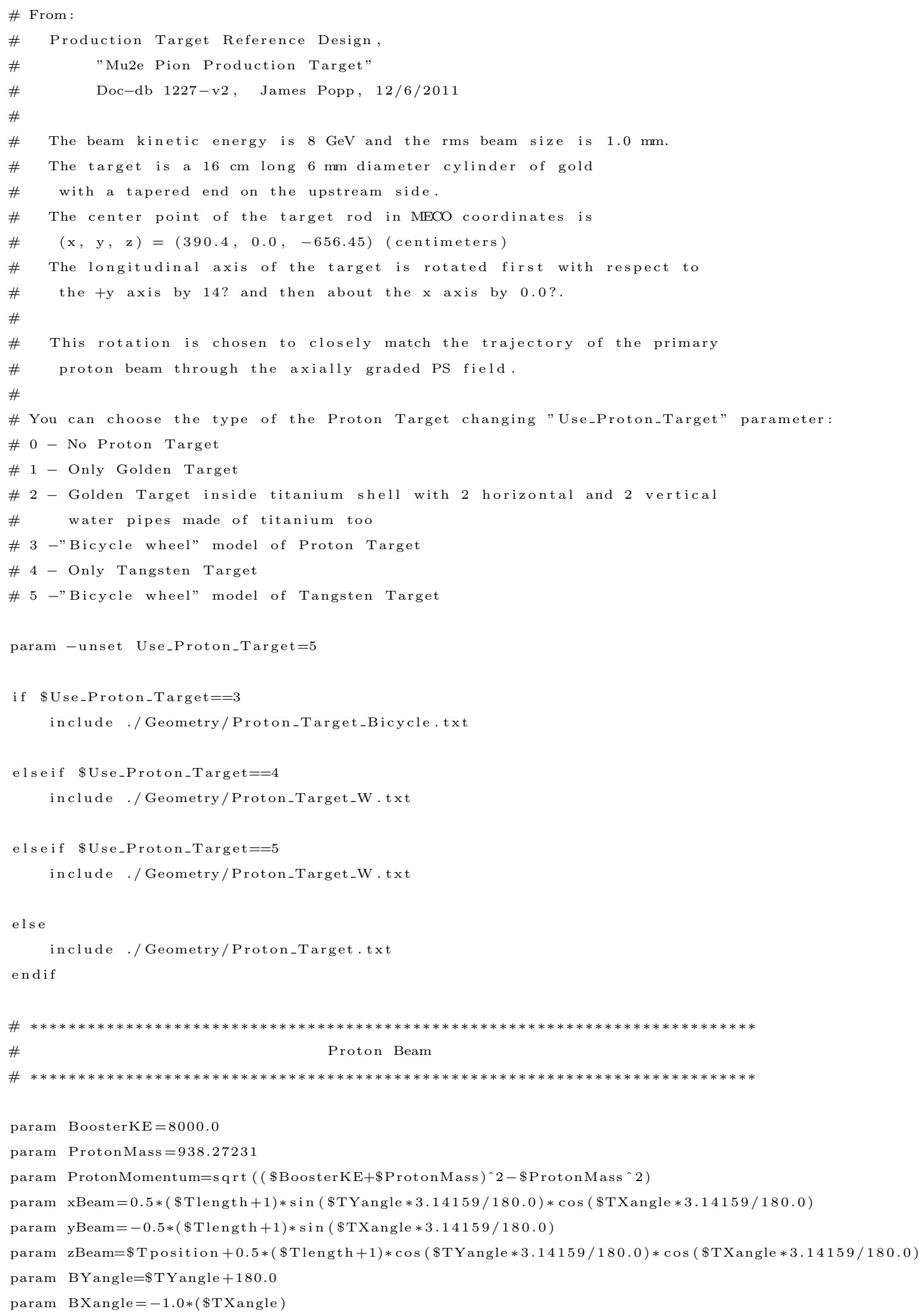




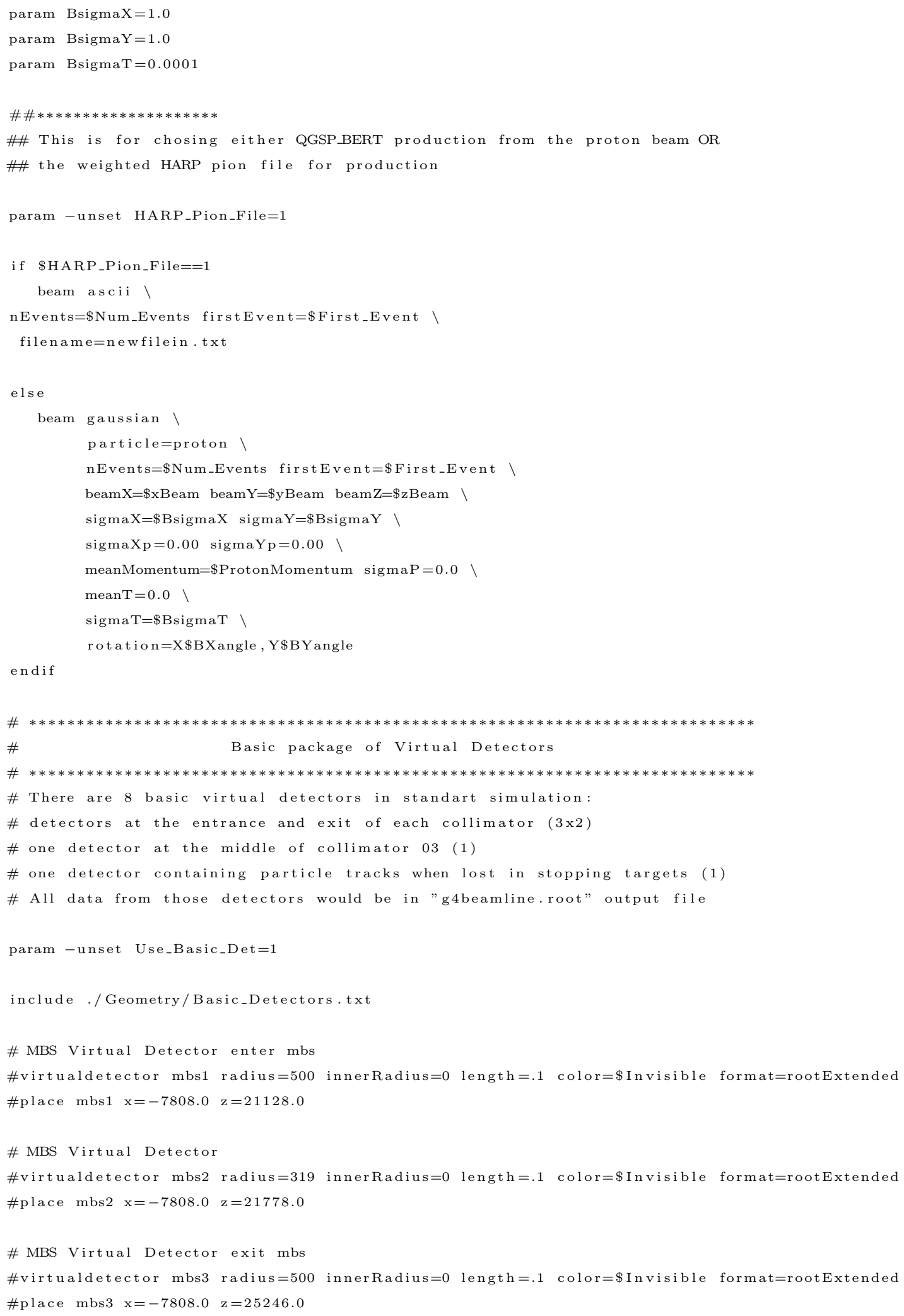




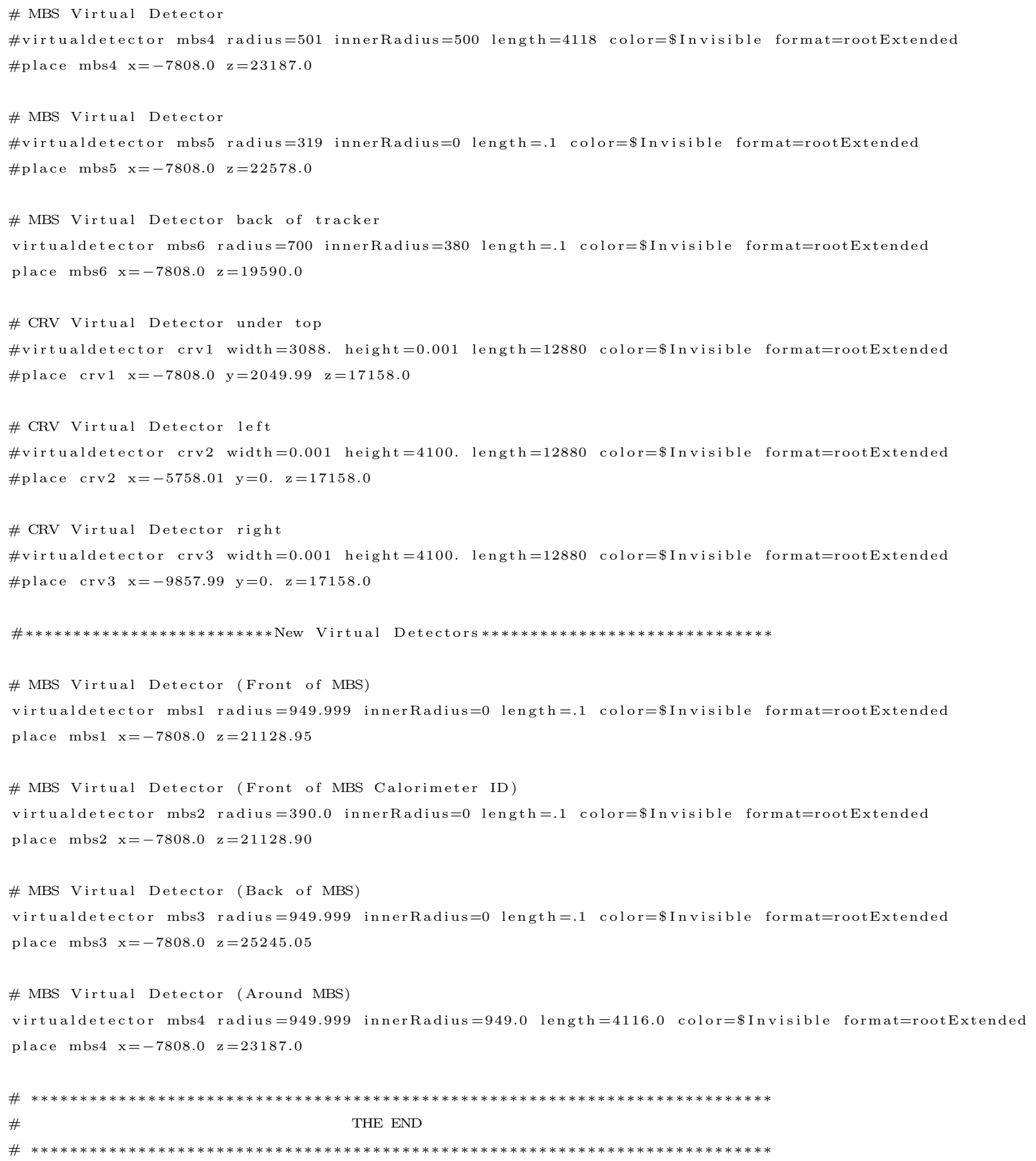


APPENDIX C

G4BEAMLINE MBS GEOMETRY FILE 


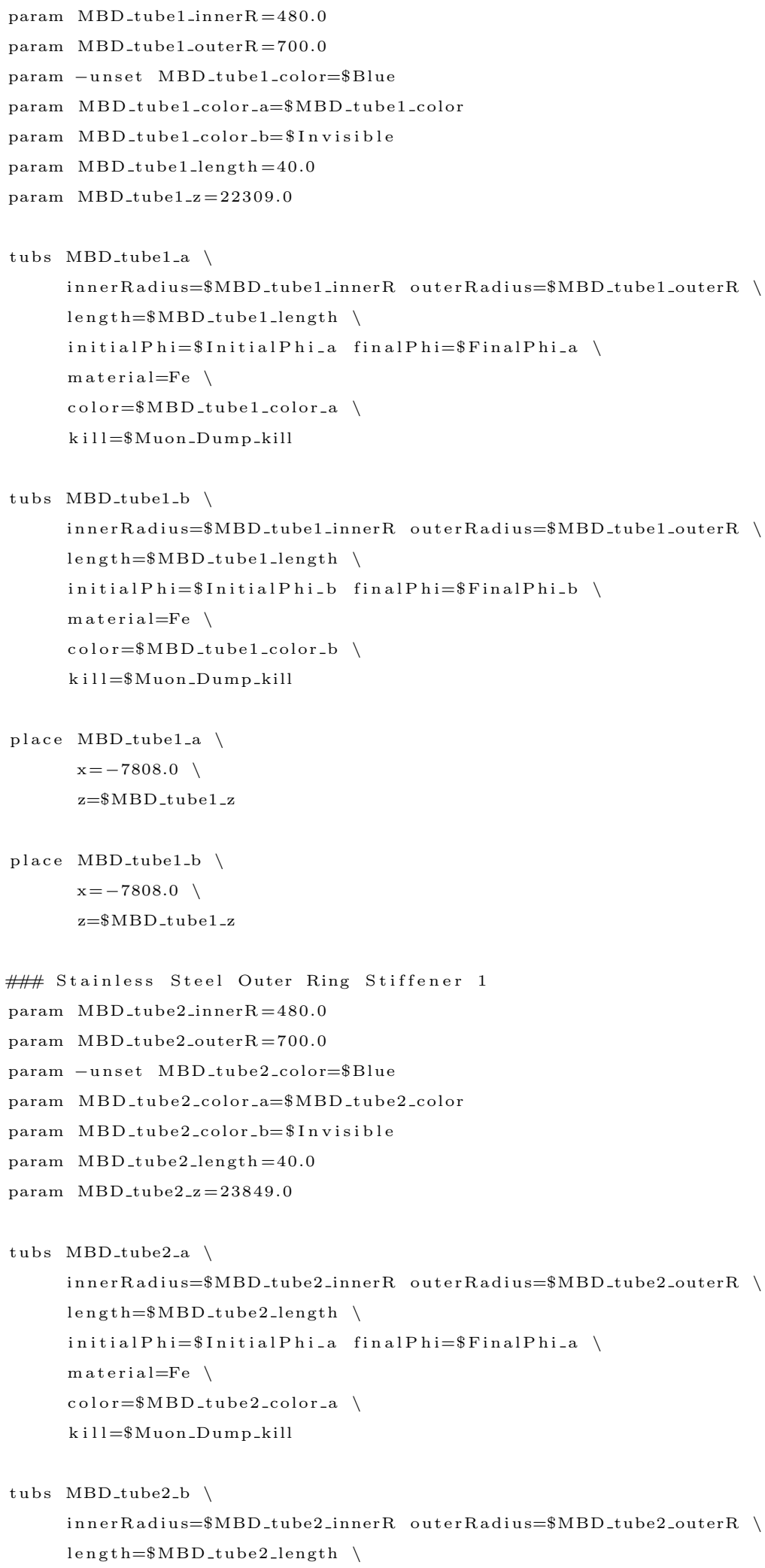




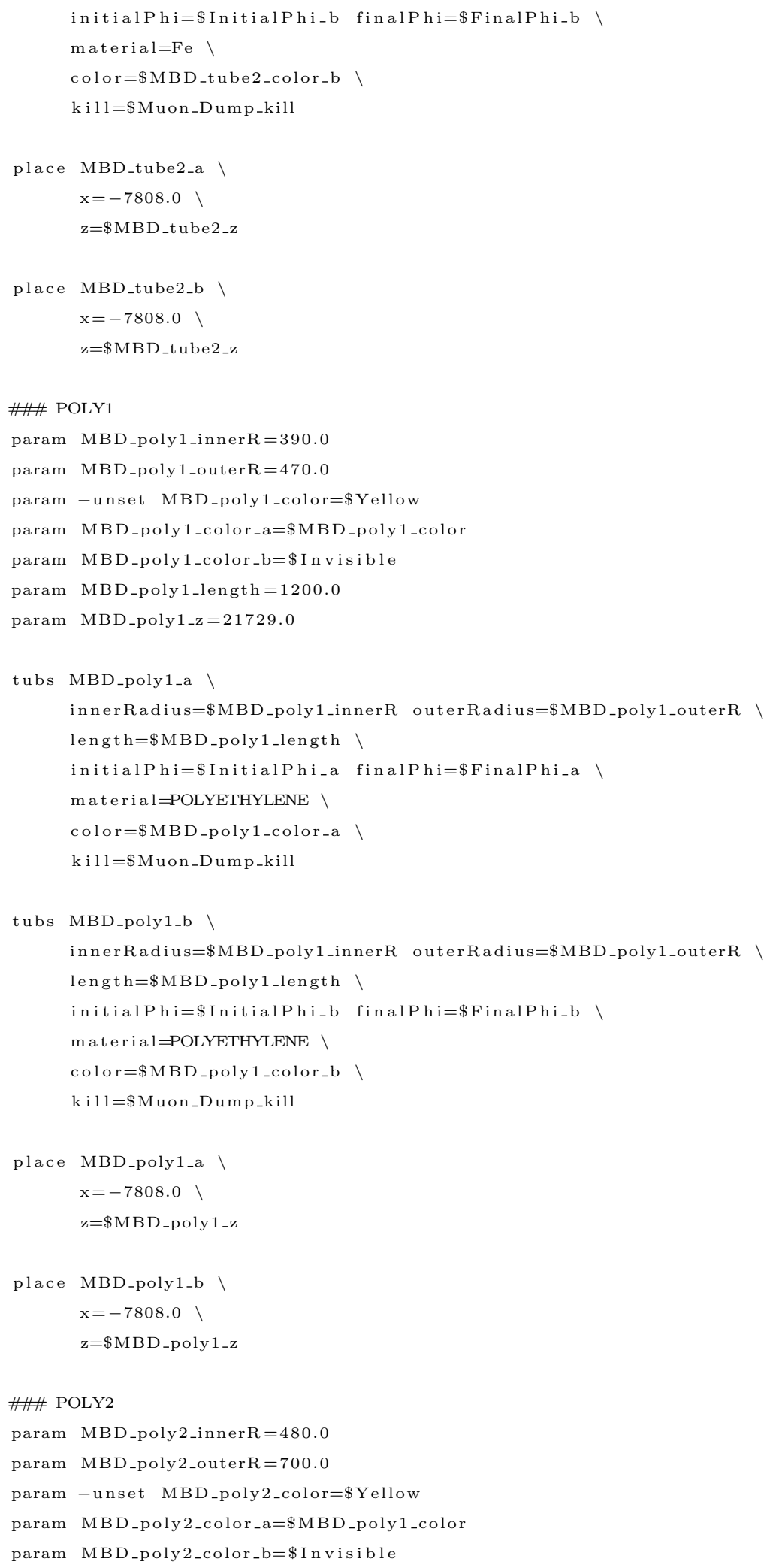




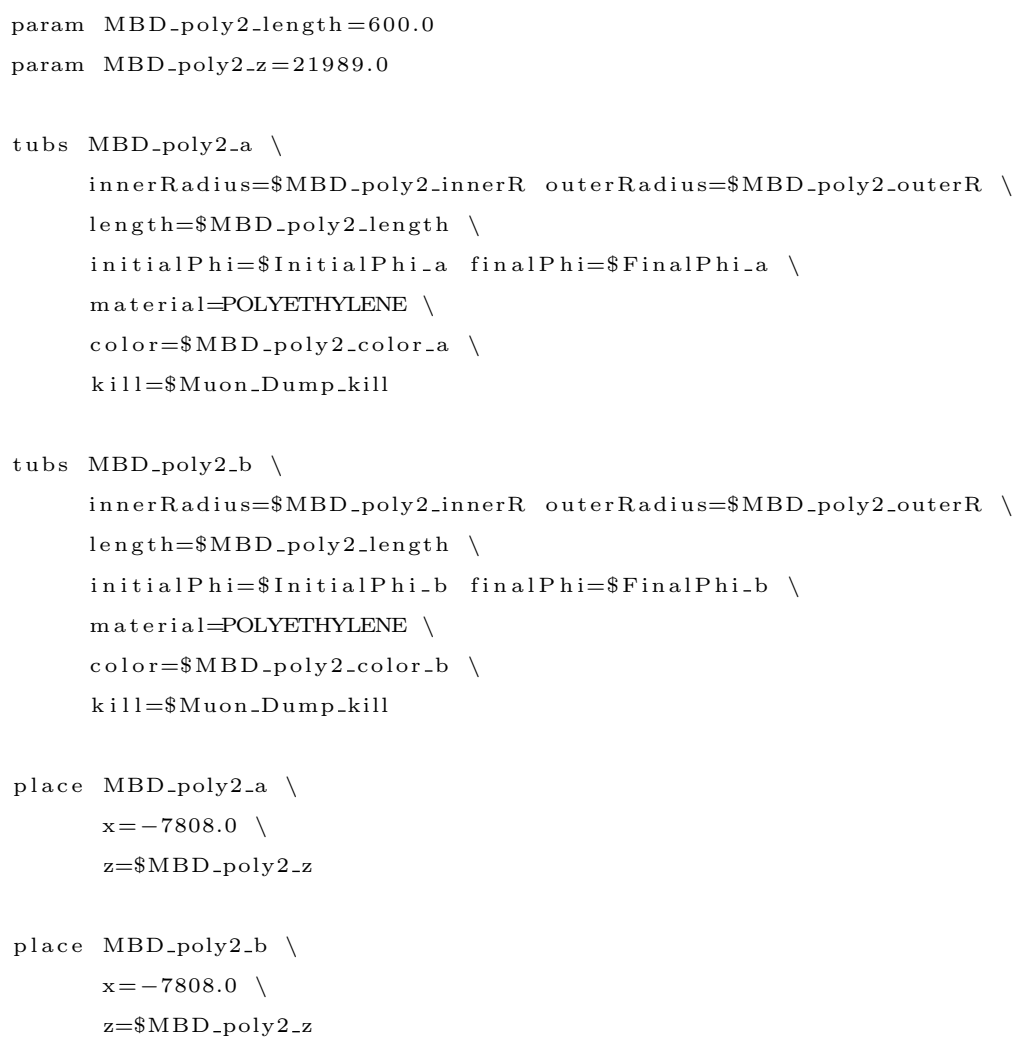




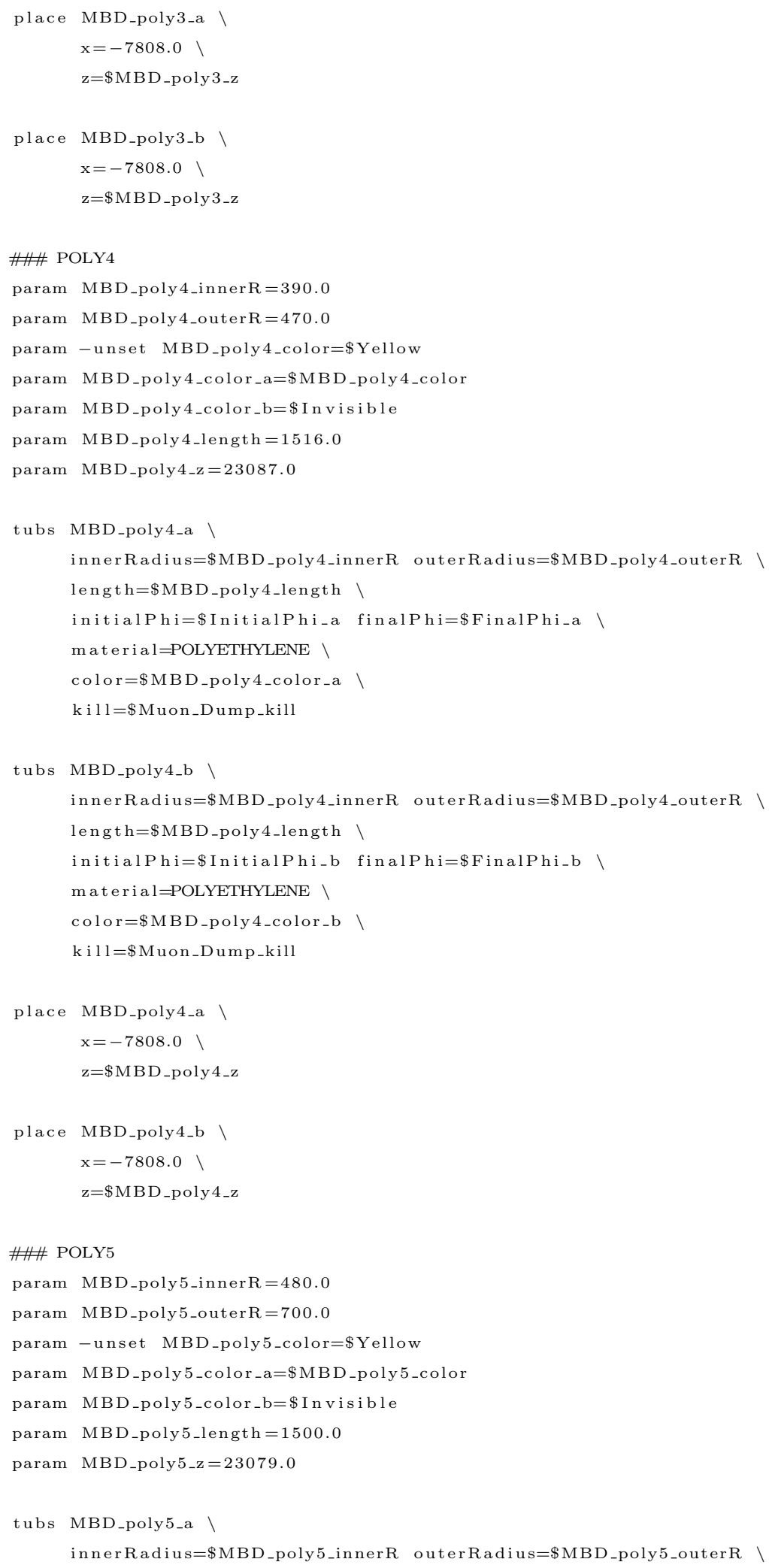




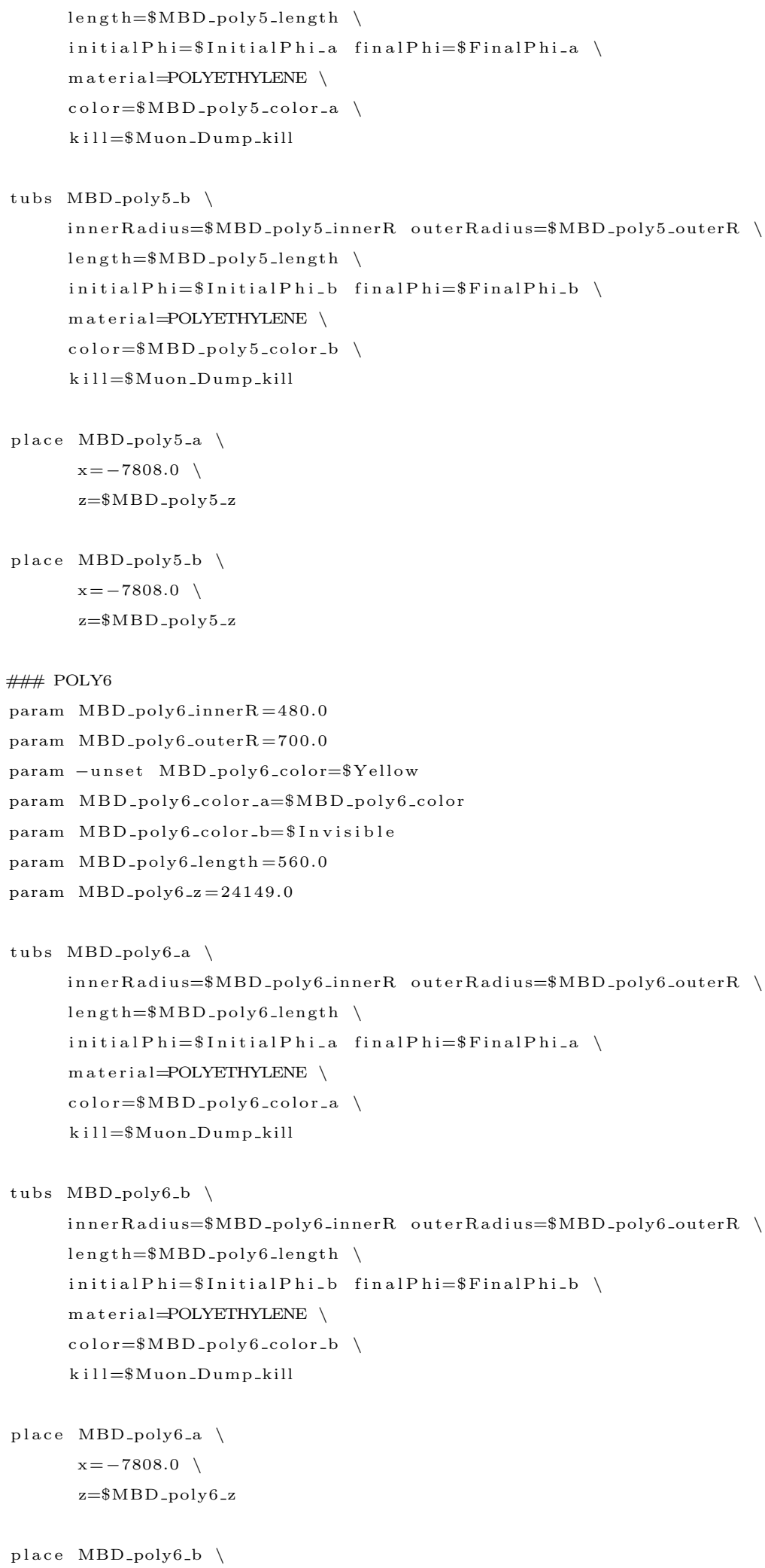




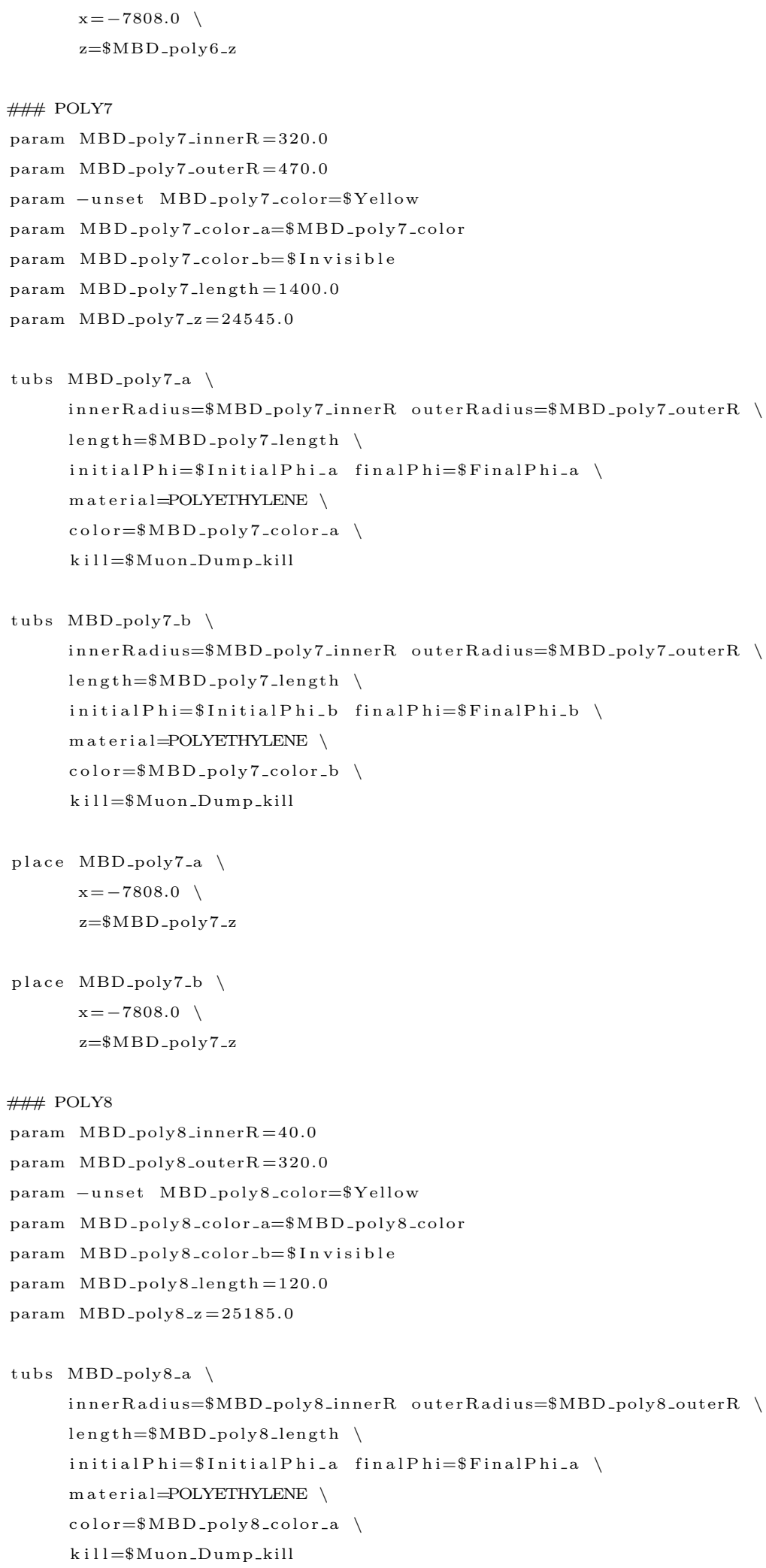




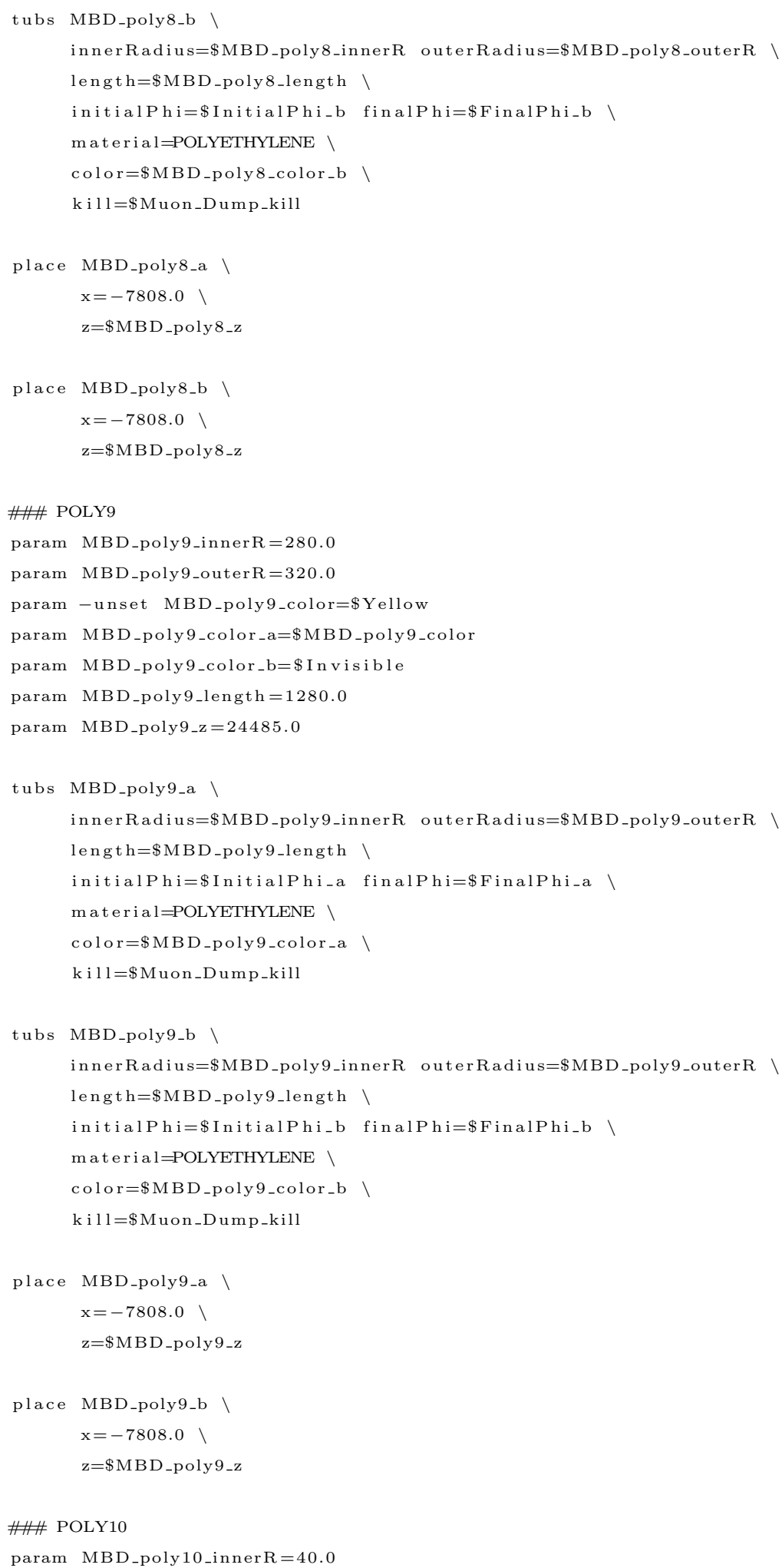




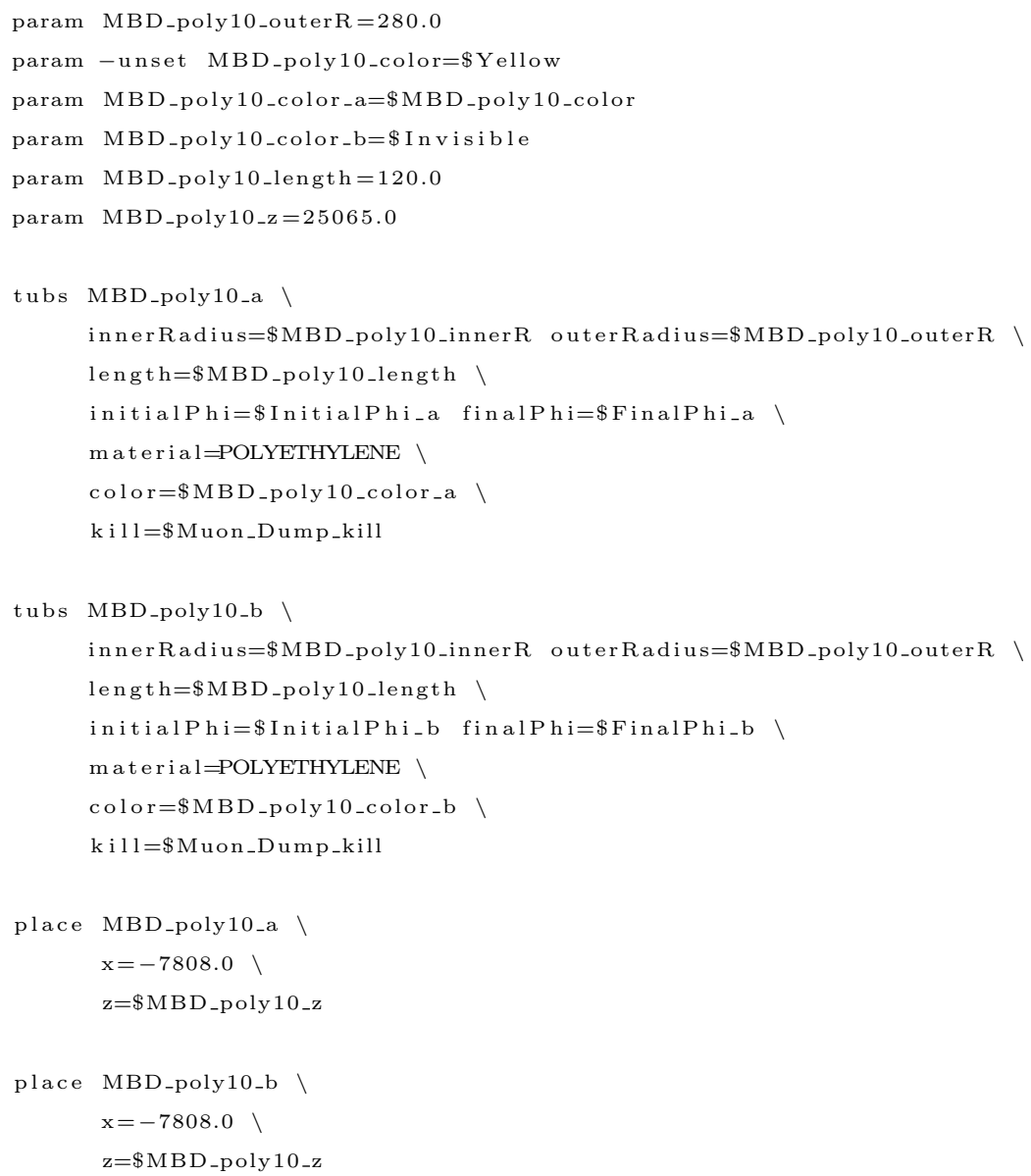


APPENDIX D

G4BEAMLINE SETUP FILE 


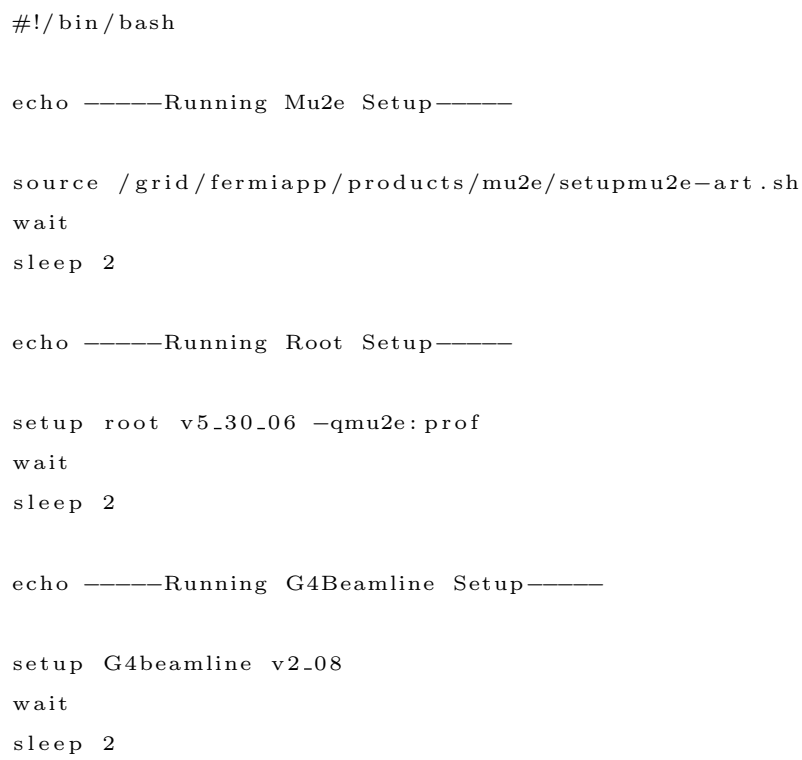


APPENDIX E

POST-PROCESSING MACRO 


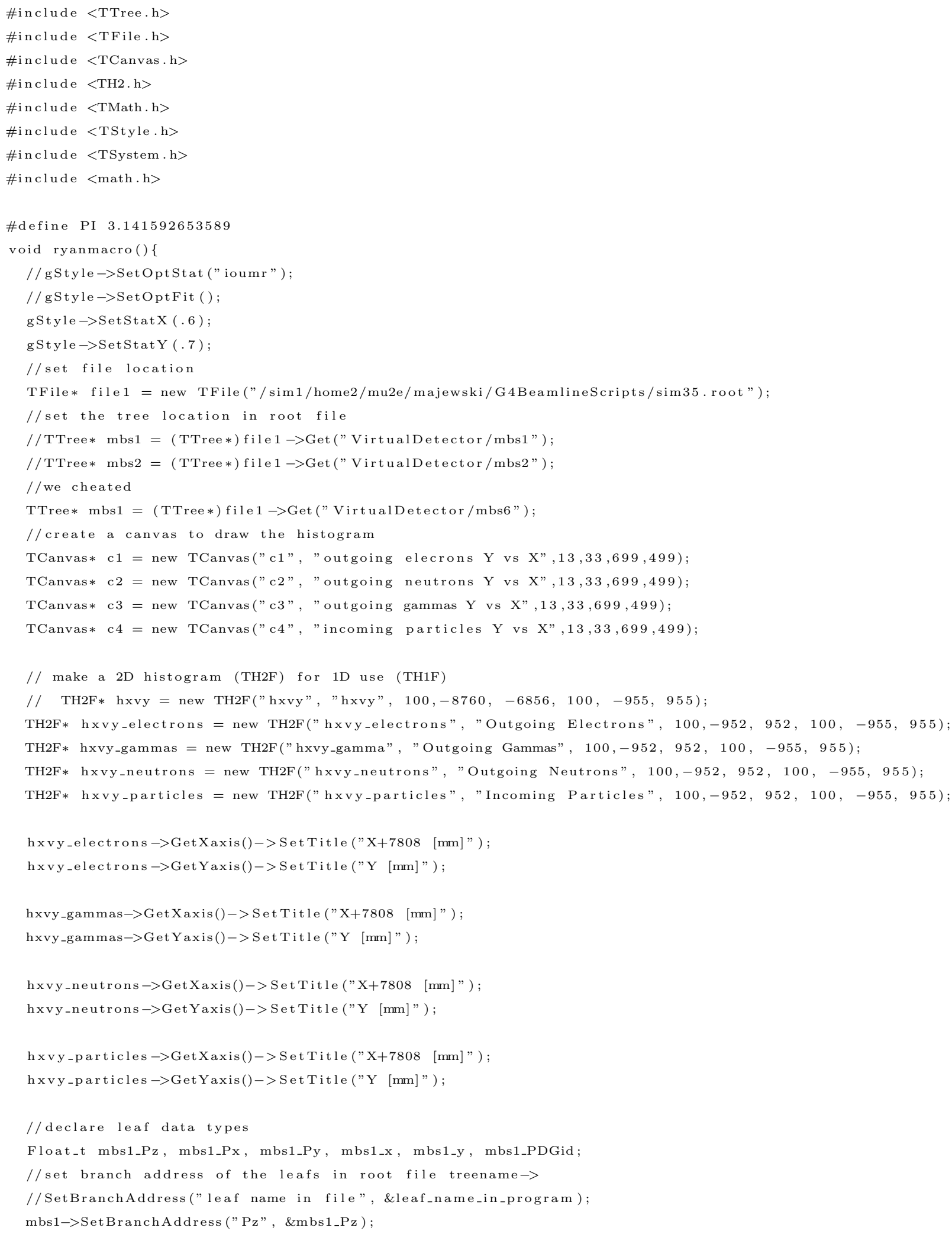




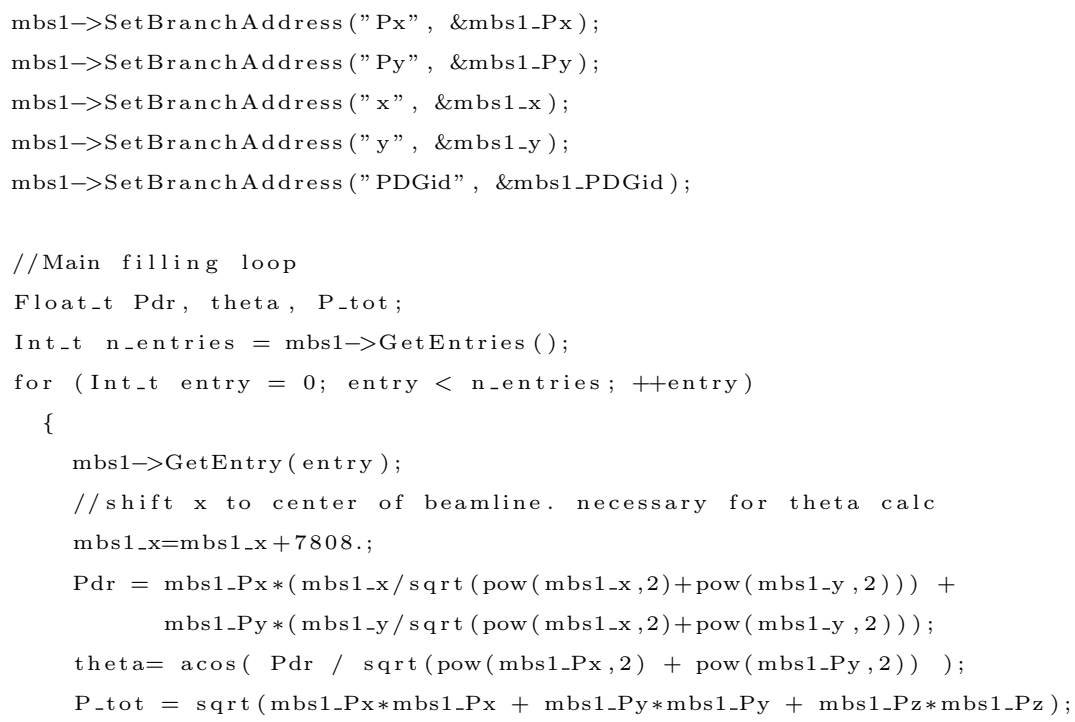




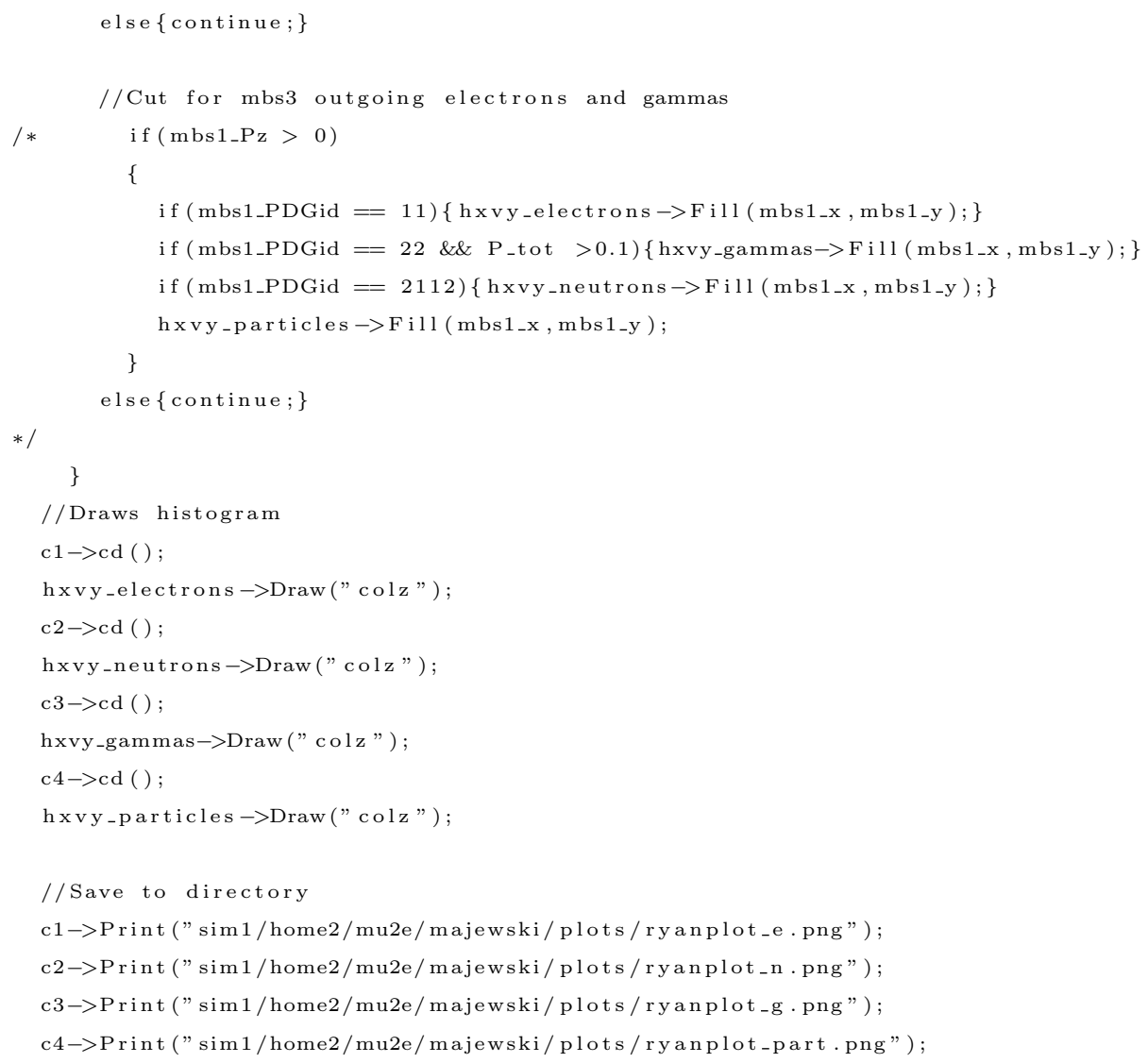

